JOURNAL OF THE

AMERICAN MATHEMATICAL SOCIETY

Volume 22, Number 4, October 2009, Pages 1157-1215

S 0894-0347(09)00639-0

Article electronically published on May 1, 2009

\title{
MINIMUM VOLUME CUSPED HYPERBOLIC THREE-MANIFOLDS
}

\author{
DAVID GABAI, ROBERT MEYERHOFF, AND PETER MILLEY
}

\section{INTRODUCTION}

In this paper, we prove:

Theorem 1.1. Let $N$ be a one-cusped orientable hyperbolic 3-manifold with $\operatorname{Vol}(N)$ $\leq 2.848$. Then $N$ can be obtained by Dehn filling all but one of the cusps of $M$, where $M$ is one of the 21 cusped hyperbolic 3-manifolds listed in the table in Figure 1.

In [M], the Dehn surgery spaces of the 21 manifolds listed in Figure 1 are rigorously analyzed, producing a complete list of one-cusped manifolds with volume no greater than 2.848 which result from the Dehn fillings described above. We therefore obtain:

Corollary 1.2. Let $N$ be a 1-cusped orientable hyperbolic 3-manifold with $\operatorname{Vol}(N)$ $\leq 2.848$. Then $N$ is one of $m 003, m 004, m 006, m 007, m 009, m 010, m 011, m 015$, $m 016$, or $m 017$. (Notation as in the SnapPea census.)

This corollary extends work of Cao and Meyerhoff who had earlier shown that m003 and m004 were the smallest-volume cusped manifolds. Also, the above list agrees with the SnapPea census of one-cusped manifolds produced by Jeff Weeks ([W]), whose initial members are conjectured to be an accurate list of small-volume cusped manifolds.

Let $N$ be a closed hyperbolic 3-manifold with simple closed geodesic $\gamma$ and let $N_{\gamma}$ denote the manifold $N \backslash \gamma$. Agol (Ago ) discovered an inequality relating $\operatorname{Vol}(N)$ to $\operatorname{Vol}\left(N_{\gamma}\right)$ and the tube radius of $\gamma$. Assuming certain results of Perelman, Agol and Dunfield (see $[\mathrm{AST}$ ) have further strengthened that result. A straightforward calculation (see [ACS] ) using this stronger result, the $\log (3) / 2$ theorem of [GMT, plus bounds on the density of hyperbolic tube packings by Przeworksi in $[\mathrm{P}$, shows that a compact hyperbolic manifold with volume less than or equal to that of the Weeks manifold must be obtainable by Dehn filling on a cusped manifold with volume less than or equal to 2.848. The paper [M] rigorously shows that the Weeks manifold is the unique compact hyperbolic 3-manifold of smallest volume obtained by filling any of the 10 manifolds listed in Corollary 1.2. We therefore obtain:

Received by the editors August 14, 2008.

2000 Mathematics Subject Classification. Primary 57M50; Secondary 51M10, 51M25.

The first author was partially supported by NSF grants DMS-0554374 and DMS-0504110.

THe second author was partially supported by NSF grants DMS-0553787 and DMS-0204311.

The third author was partially supported by NSF grant DMS-0554624 and by ARC Discovery grant DP0663399. 


\begin{tabular}{|c|c|c|c|c|c|c|}
\hline \hline $\mathrm{m} 125$ & $\mathrm{~m} 129$ & $\mathrm{~m} 203$ & $\mathrm{~m} 202$ & $\mathrm{~m} 292$ & $\mathrm{~m} 295$ & $\mathrm{~m} 328$ \\
$\mathrm{~m} 329$ & $\mathrm{~m} 359$ & $\mathrm{~m} 366$ & $\mathrm{~m} 367$ & $\mathrm{~m} 391$ & $\mathrm{~m} 412$ & $\mathrm{~s} 596$ \\
$\mathrm{~s} 647$ & $\mathrm{~s} 774$ & $\mathrm{~s} 776$ & $\mathrm{~s} 780$ & $\mathrm{~s} 785$ & $\mathrm{~s} 898$ & $\mathrm{~s} 959$ \\
\hline \hline
\end{tabular}

Figure 1. The cusped manifolds which generate all one-cusped hyperbolic 3 -manifolds with volume $\leq 2.848$. The manifolds are denoted here as they appear in the SnapPea census.

Corollary 1.3. The Weeks manifold is the unique closed orientable hyperbolic 3manifold of smallest volume.

The Weeks manifold is obtained by $(5,1),(5,2)$ filling on the Whitehead link, or by $(2,1)$ filling on the manifold m003 in the SnapPea census. It was discovered and its volume was computed independently by Matveev and Fomenko in [MF] and Weeks using his SnapPea program [W]. Independently, Przytycki asked whether this manifold was the smallest closed manifold; see [BPZ] for related questions.

The proof of Theorem 1.1 is based on the Mom technology introduced in GMM2]. Indeed, Figure 1 lists the collection of Mom-2 and Mom-3 manifolds enumerated in GMM2; thus we have the following equivalent formulation:

Theorem 1.4. Let $N$ be a one-cusped orientable hyperbolic 3-manifold with $\operatorname{Vol}(N)$ $\leq 2.848$. Then $N$ can be obtained by Dehn filling all but one of the cusps of $M$, where $M$ is a hyperbolic Mom-2 or Mom-3 manifold.

Recall that a Mom- $n$ manifold is a 3 -manifold $M$ obtained by starting with $T \times[0,1]$, where $T$ is the 2 -torus, and attaching $n$ 1-handles and $n$ valence- 32 handles to the $T^{2} \times 1$ side. Furthermore, $\partial M$ is a union of tori. Given $N$ as in the theorem, the goal is to find a hyperbolic Mom-3 embedded in $N$, or in the terminology of GMM2, show that $N$ possesses an internal Mom-n structure for some $n \leq 3$. That is, $M$ satisfies the condition that the interior of $M$ has a complete hyperbolic structure of finite volume and each component of $\partial M$ bounds (to the outside) either a solid torus or a cusp.

In practice, we think of $T \times 0$ as the torus cutting off a maximal cusp neighborhood $U$. In the universal covering, $U$ lifts to a collection of horoballs $\left\{B_{i}\right\}$. To a first approximation, when lifted to $\mathbb{H}^{3}$, the cores of the 1-handles of the Mom- $n$ structure will be geodesic arcs connecting two $B_{i}$ 's. (Being maximal, some $B_{i}$ 's will be tangent to each other and these points of tangency will also be viewed as 1-handles.) The cores of the 2-handles, when lifted to $\mathbb{H}^{3}$ will correspond to totally geodesic hexagons whose sides alternately lie on the 1-handles and boundaries of the $B_{i}$ 's.

Using the 2.848 volume bound we will show that $N$ possesses a geometric Mom- $n$ structure, where $n \leq 3$. This means that we will find $n \pi_{1}(N)$-orbits of geodesic arcs and $n \pi_{1}(N)$-orbits of geodesic hexagons with boundaries on the geodesic arcs and $B_{i}$ 's as in the previous paragraph. With some luck, when thickened up, these geodesics and hexagons will descend to an internal Mom- $n$ structure on $N$. In reality, when projected to $N$, these hexagons may self-intersect in undesirable ways and/or the resulting handle structures may be unsuitable for various technical reasons. Much of this paper is devoted to the process of promoting a geometric Mom- $n$ structure for $n \leq 3$ which is torus-friendly to a hyperbolic internal Mom- $k$ structure, $k \leq n$. (See Definition 2.2.) 
This paper is organized as follows. In Section 2 we give a detailed definition of geometric Mom- $n$ structure. In Section 3 we present several useful geometric lemmas that will be used extensively in the rest of the paper. Then in Sections 4 and 5 we show that if $N$ satisfies the hypotheses of Theorem 1.1, then $N$ must contain a geometric Mom- $n$ structure which is torus-friendly. This part of the proof, while theoretically simple, is computationally complicated and was completed with the use of computer assistance; the use of rigorous floating-point computations is discussed in Section 5.

Sections 6,7 , and 8 are concerned with the process of promoting the geometric Mom- $n$ structure produced in Sections 4 and 5 to an internal Mom- $n$ structure of the type described in GMM2. There is a natural geometric object associated to a geometric Mom- $n$ structure, consisting of a thickened copy of the cusp torus $\partial U$ (which corresponds to our $\left.T^{2} \times[0,1]\right)$ together with a "1-handle" for every orthogonal geodesic arc in the Mom- $n$ structure and a "2-handle" for every hexagon. However there are three key conditions that this geometric object must meet before it fits the definition of an internal Mom- $n$ structure. Each of Sections 6, 7, and 8 is devoted to one of these three conditions and to showing that either the condition holds or else we can replace our geometric Mom- $n$ with a "simpler" structure, for some appropriate definition of "simpler". Section 6 is concerned with whether or not the geometric object associated to the Mom- $n$ is embedded in $N$, in particular whether the various handles have undesirable intersections or self-intersections. Section 7 is concerned with whether the components of the complement have the correct topology, and Section 8 is concerned with whether or not our Mom- $n$ structure has "simply connected lakes" in the language of Matveev. Having completed these three sections, we find that the geometric Mom- $n$ structure produced in Sections 4 and 5 will have evolved into an internal Mom- $k$ structure for some $k \leq n$.

At that point, the proof of Theorem 1.1 reduces to an application of Theorems 4.1 and 5.1 of GMM2]. Together those two theorems imply that if $N$ has an internal Mom- $n$ structure with $n \leq 3$, then $N$ contains an embedded submanifold $M$ which is a hyperbolic manifold with boundary whose interior is homeomorphic to one of the manifolds in Figure 1 and such that $N-M$ is a disjoint union of solid tori and cusps. This is the desired result. Section 9 summarizes this argument formally.

It should be noted that while the list in Figure 1 is precisely the list of manifolds produced by Theorem 5.1 of GMM2, it is somewhat redundant for the purposes of Theorem 1.1 of this paper. The manifold s776 is a three-cusped manifold from which many of the two-cusped manifolds on the list (experimentally, everything up to and including m391) can be recovered by Dehn filling. Hence the 21 manifolds in Figure 1 could be reduced to a list of 9 manifolds with no effect on Theorem 1.1. However, we use the longer list here to be consistent with GMM2].

Finally in Section 9 we will discuss some of the ideas used in $[\mathrm{M}$ to rigorously analyze various Dehn fillings of the manifolds of Figure 1.

It should be noted that the methods of this paper can also be used to classify one-cusped manifolds with low maximal cusp volume. See Remark 5.10.

\section{Definition of a Geometric Mom- $n$}

For the rest of this paper, $N$ will refer to an orientable one-cusped hyperbolic 3 -manifold. Suppose $N$ is such a manifold; then $N$ possesses a maximal cusp neighborhood which is a closed set whose interior is homeomorphic to $T^{2} \times(0, \infty)$, 
with the property that each torus $T^{2} \times\{x\}$ has constant sectional curvature in $N$. The term "maximal" here means that this cusp neighborhood is not a proper subset of any other closed subset of $N$ with this property.

$T$ bounds a horoball when lifted to $\tilde{T}$ in the universal covering $\mathbb{H}^{3}$ of $N$. In practice we think of $T$ as a maximal cusp bounding a horoball. In $\mathbb{H}^{3}$, the cores of the various 1-handles will be orthogonal geodesic arcs (or points) connecting $\pi_{1}(N)$ translates of this horoball. The cores of the 2-handles will be totally geodesic discs with boundary alternately on horoballs and these geodesic arcs.

We prove Theorem 1.1 using geometric Mom-n structures, defined below.

In the universal cover $\mathbb{H}^{3}$ of $N$, the maximal cusp neighborhood lifts to a collection of horoballs $\left\{B_{i}\right\}$; any two such horoballs have disjoint interiors, but maximality implies that some pairs will be tangent at their boundaries. Choose one such horoball and denote it by $B_{\infty}$. In the upper half-space model $\{(x, y, z) \mid z>0\}$ of $\mathbb{H}^{3}$, we may assume after conjugation by some element of $\operatorname{Isom}^{+}\left(\mathbb{H}^{3}\right)$ that $B_{\infty}$ will be precisely the set $\{(x, y, z) \mid z \geq 1\}$; then every other $B_{i}$ will appear as a sphere with center $\left(x_{i}, y_{i}, z_{i}\right)$ and radius $z_{i}$ for some $0<z_{i} \leq \frac{1}{2}$. Let $H \subset \pi_{1}(N)$ be the subgroup which fixes $B_{\infty}$, so that $B_{\infty} / H$ is homeomorphic to the cusp neighborhood. Let $d_{E}$ denote the distance function in the subspace (Euclidean) metric along the boundary of $B_{\infty}$.

The center of a horoball $B_{j} \neq B_{\infty}$ is the limiting point of the horoball on the sphere at infinity. For example, if $B_{j}$ appears in the upper half-space model as a sphere centered at $\left(x_{j}, y_{j}, z_{j}\right)$ with radius $z_{j}$, then the center of the horoball is the point $\left(x_{j}, y_{j}, 0\right)$. We will sometimes refer to the complex number $x_{j}+i y_{j}$ as the center of $B_{j}$ in this case, and we define the center of $B_{\infty}$ to be $\infty$; then the center of a horoball is always an element of $\hat{\mathbb{C}}=\mathbb{C} \cup\{\infty\}$.

In addition to this we will define the orthocenter of a horoball $B_{j} \neq B_{\infty}$ to be the point on $\partial B_{\infty}$ which is closest to $B_{j}$. Uniqueness is guaranteed by the fact that the boundary of any horoball has positive sectional curvature in $\mathbb{H}^{3}$, being a Euclidean surface in a negatively curved space. In the upper half-space model, the orthocenter of $B_{j}$ is just the point on the surface $z=1$ directly above the center of $B_{j}$.

Given two horoballs $A$ and $B$, neither equal to $B_{\infty}$, we will say that $A$ and $B$ are in the same orthoclass if either $A$ and $B$ lie in the same $H$-orbit or there exists some $g \in \pi_{1}(N)$ such that $g(A)=B_{\infty}$ and $g\left(B_{\infty}\right)=B$. In the latter case we say that $A$ and $B$ lie in conjugate $H$-orbits. We denote the orthoclasses by $\mathcal{O}(1), \mathcal{O}(2)$, and so forth. For any $B \in \mathcal{O}(n)$ we call $d\left(B, B_{\infty}\right)$ the orthodistance and denote it by $o(n)$; this is clearly well defined. Order the orthoclasses $\mathcal{O}(1), \mathcal{O}(2), \ldots$ in such a way that the corresponding orthodistances are non-decreasing: $0=o(1) \leq o(2) \leq \cdots$. We will refer to this as the orthodistance spectrum. In addition we also define $e_{n}=\exp (o(n) / 2)$, and refer to the sequence $1=e_{1} \leq e_{2} \leq \cdots$ as the Euclidean spectrum. Note that if $A \in \mathcal{O}(n)$, then in the upper half-space model the point on $\partial A$ which is closest to $B_{\infty}$ must appear to be at a height of $\exp \left(-d\left(A, B_{\infty}\right)\right)$, which equals $e_{n}{ }^{-2}$ since $d\left(A, B_{\infty}\right)=o(n)$. The choice of the word "Euclidean" actually comes from Lemma 3.4 .

Closely related to the orthoclasses are another set of equivalence classes which we will call the orthopair classes. These are just the equivalence classes of the action of $\pi_{1}(N)$ on the set of unordered pairs of horoballs $\{A, B\}$. It follows immediately from the definition that $A$ and $B$ are in the same orthoclass if and only if $\left\{A, B_{\infty}\right\}$ and 
$\left\{B, B_{\infty}\right\}$ lie in the same orthopair class. Hence we will occasionally abuse notation and denote the orthopair classes by $\mathcal{O}(1), \mathcal{O}(2), \ldots$ as well. The definition of orthodistance in this context is clear.

Definition 2.1. A $(p, q, r)$-triple (or equivalently a triple of type $(p, q, r))$ is a triple of horoballs $\left\{B_{1}, B_{2}, B_{3}\right\}$ with the property that $\left\{B_{1}, B_{2}\right\} \in \mathcal{O}(p),\left\{B_{2}, B_{3}\right\} \in \mathcal{O}(q)$, and $\left\{B_{3}, B_{1}\right\} \in \mathcal{O}(r)$, possibly after reordering.

Now we come to the key definition of this paper.

Definition 2.2. A geometric Mom-n structure is a collection of $n$ triples of types $\left(p_{1}, q_{1}, r_{1}\right), \ldots,\left(p_{n}, q_{n}, r_{n}\right)$, no two of which are equivalent under the action of $\pi_{1}(N)$, and such that the indices $p_{i}, q_{i}$, and $r_{i}$ all come from the same $n$-element subset of $\mathbb{Z}_{+}$.

We will occasionally drop the word "structure" when our meaning is otherwise clear.

A geometric Mom- $n$ will be said to be torus-friendly if $n=2$ or if $n=3$ and the Mom-3 does not possess exactly two triples of type $(p, q, r)$ for any set of distinct positive indices $p, q$, and $r$. (The geometrical implications of this term will be explained in Section 7.)

So, for example, a $(1,1,3)$-triple and a $(1,3,3)$-triple would constitute a geometric Mom-2, while a $(1,1,2)$-triple and a (1,1,3)-triple would not. A (1,1,2)-triple, a (1,1,3)-triple, and a (1,2,3)-triple, however, would constitute a geometric Mom-3 which furthermore is torus-friendly. A (1,1,2)-triple and two $(1,2,3)$-triples which are not equivalent under the action of $\pi_{1}(M)$ form a geometric Mom-3 which is not torus-friendly. Although this definition can clearly be generalized, in this paper we will only be discussing geometric Mom- $n$ 's where $n=2$ or 3 , and where the indices all come from the set $\{1,2,3,4\}$.

The connection between geometric Mom- $n$ 's and the internal Mom- $n$ structures of GMM2 is clear, although the definition of geometric Mom- $n$ structures does not include any of the topological assumptions that are part of the definition of an internal Mom- $n$ structure, such as embeddedness. Nevertheless, the correspondence between geometric and internal Mom- $n$ structures is the key to proving Theorem 1.1 .

\section{Geometrical lemmas}

Throughout this chapter we will be using the upper half-space model of $\mathbb{H}^{3}$. We will take certain facts, listed below, as given; a reader who is interested may refer to Fen for more information.

First, the orientation-proving isometries of $\mathbb{H}^{3}$ can be identified with the matrix group $\operatorname{PSL}(2, \mathbb{C})$ in a natural way. Each element of $\operatorname{PSL}(2, \mathbb{C})$ acts on the sphere at infinity $\hat{\mathbb{C}}=\mathbb{C} \cup\{\infty\}$ by the corresponding Mobius transformation, i.e.

$$
\left[\begin{array}{ll}
a & b \\
c & d
\end{array}\right]: z \mapsto \frac{a z+b}{c z+d}
$$

if $z \in \mathbb{C}$, and $\infty \mapsto \frac{a}{c}$. The action of $\operatorname{PSL}(2, \mathbb{C})$ on $\mathbb{H}^{3}$ itself can be expressed similarly, using quaternions. In the upper half-space model, the point $(x, y, t)$ in $\mathbb{H}^{3}$ with $t>0$ can be associated to the quaternion $x+y i+t j$; then the action of 
$\operatorname{PSL}(2, \mathbb{C})$ can be expressed as

$$
\left[\begin{array}{ll}
a & b \\
c & d
\end{array}\right]: x+y i+t j \mapsto(a(x+y i+t j)+b)(c(x+y i+t j)+d)^{-1} .
$$

The resulting quaternion will always be equal to $u+v i+s j$ for some real $(u, v, s)$ with $s>0$.

If $g \in \operatorname{PSL}(2, \mathbb{C})$ is not the identity, then we can determine from the trace of $g$ whether or not $g$ is hyperbolic, parabolic, or elliptic as an isometry (note that trace is only defined up to sign in $\operatorname{PSL}(2, \mathbb{C})$ ). For example, $g$ is an elliptic isometry (that is, $g$ is a rotation about a line in $\mathbb{H}^{3}$ ) if and only if $\operatorname{tr}(g)= \pm 2 \cos (\theta / 2)$, where $\theta \in[-\pi, \pi]$ is the angle of rotation of $g$. Note that in contrast to the hyperbolic and parabolic cases, an elliptic isometry cannot be an element of $\pi_{1}(N)$, where $N$ is a 1-cusped hyperbolic 3 -manifold.

One final fact about $\operatorname{PSL}(2, \mathbb{C})$ that we will use: if $g=\left[\begin{array}{ll}a & b \\ c & d\end{array}\right]$, then the image of $B_{\infty}$ under $g$ will be a horoball which appears as a Euclidean ball of diameter $|c|^{-2}$. More generally, the subset $B(t)=\{(x, y, z) \mid z \geq t\}$ of $\mathbb{H}^{3}$ will be mapped to a horoball which appears as a Euclidean ball of diameter $t^{-1}|c|^{-2}$. This can be demonstrated as follows. Since $g(\infty)=\frac{a}{c}$, the image of $B(t)$ will be a horoball with center $\frac{a}{c} \in \mathbb{C}$. Therefore the point of $g(B(t))$ of greatest apparent height will correspond to the quaternion $\frac{a}{c}+\delta j$, where $\delta$ is the diameter we seek. This is just the point where $g(B(t))$ intersects the line in $\mathbb{H}^{3}$ from $\infty$ to $\frac{a}{c}$. Therefore the preimage of this point under $g$ is the point where $B(t)$ intersects the line from $g^{-1}(\infty)=-\frac{d}{c}$ to $g^{-1}\left(\frac{a}{c}\right)=\infty$. In other words,

$$
\frac{a}{c}+\delta j=g\left(-\frac{d}{c}+t j\right) .
$$

Direct calculation with quaternions then yields the desired result.

We now begin enumerating the geometrical lemmas that we will use in the rest of the paper. The following lemma first appears (using different language) in [Ada].

Lemma 3.1. Every orthoclass consists of two H-orbits.

Proof. It is fairly clear from the definition that each orthoclass contains no more than two $H$-orbits. If an orthoclass contains exactly one $H$-orbit, then we must have $g^{-1}\left(B_{\infty}\right)=h g\left(B_{\infty}\right)$ for some $h \in H, g \notin H$. In other words, $g h g \in H$, and therefore $(g h)^{2} \in H$. But as an isometry, $g h$ must be either hyperbolic, parabolic, or the identity. In the first case, $(g h)^{2}$ would also be hyperbolic (and hence not in $H$ ), and in the last two cases, we would have $g h \in H$ and hence $g \in H$. Either case is a contradiction.

The following lemma appears in [CM]; a related lemma for compact manifolds appears in GMM]. It is reproduced here for the sake of completeness.

Lemma 3.2. If $A$ and $B$ are both in $\mathcal{O}(n)$, and if $g \in \pi_{1}(N)$ is an isometry such that $g(B)=B_{\infty}$, then $g(A) \notin \mathcal{O}(n)$.

Proof. There are two cases to consider: either $A$ and $B$ lie in different $H$-orbits or else they lie in the same $H$-orbit.

In the first case, by the definition of an orthoclass, $g\left(B_{\infty}\right)=h(A)$ for some $h \in H$. By replacing $g$ with $h^{-1} g$, therefore, we may assume that $g\left(B_{\infty}\right)=A$. Suppose that $g(A)$ is in $\mathcal{O}(n)$. Then either $g(A)=h_{1}(B)$ for some $h_{1} \in H$, in 
which case we have $g^{2}\left(B_{\infty}\right)=h_{1} g^{-1}\left(B_{\infty}\right)$, or else $g(A)=h_{1}(A)$, in which case we have $g^{2}\left(B_{\infty}\right)=h_{1} g\left(B_{\infty}\right)$.

Either way we have $g^{2}=h_{1} g^{ \pm 1} h_{2}$ for some $h_{2} \in H$. Let

$$
g=\left[\begin{array}{ll}
a & b \\
c & d
\end{array}\right], h_{i}=\left[\begin{array}{cc}
1 & k_{i} \\
0 & 1
\end{array}\right], i \in\{1,2\} .
$$

Expanding both sides of the equation $g^{2}=h_{1} g^{ \pm 1} h_{2}$ and taking the $(2,1)$-entry of the resulting matrix on each side, we get $c(a+d)= \pm c$. Therefore the square of the trace of $g$ is 1 , which implies that $g \in \pi_{1}(N)$ is elliptic of order 3, a contradiction.

The other case is when $A$ and $B$ lie in the same $H$-orbit, i.e. $A=h(B)$ for some $h \in H$. Suppose that $g(A) \in \mathcal{O}(n)$ and that $g(A)$ lies in the same $H$ orbit as $A$ and $B$, i.e. $g(A)=k(B)$ for some $k \in H$. Then $g(A)$ and $g\left(B_{\infty}\right)$ both lie in $\mathcal{O}(n)$ but must lie in different $H$-orbits (see Lemma 3.11). Furthermore $g k^{-1}(g(A))=g(B)=B_{\infty}$ and $g k^{-1}\left(B_{\infty}\right)=g\left(B_{\infty}\right)$. Therefore replacing $B$ with $g(A), A$ with $g\left(B_{\infty}\right)$, and $g$ with $g k^{-1}$ reduces the problem to the previous case. On the other hand, suppose $g(A) \in \mathcal{O}(n)$ but $g(A)$ does not lie in the same $H$-orbit as $A$ and $B$. Then $g(A)=k g\left(B_{\infty}\right)$ for some $k \in H$. Since $A=h(B)=h g^{-1}\left(B_{\infty}\right)$, we have $g h g^{-1}=k g l$ for some $l \in H$. This last equation, after some manipulation (note that $h$ and $l$ commute), implies that $k^{-1}(g l) h=(g l)^{2}$, which leads to a contradiction just as in the first case. This completes the proof of the lemma.

Another way of phrasing the above result is to say that the unordered pair $\{A, B\}$ does not lie in the same orthopair class as the pairs $\left\{A, B_{\infty}\right\}$ and $\left\{B, B_{\infty}\right\}$. Hence we immediately get the following:

Corollary 3.3. There are no $(n, n, n)$-triples.

The following lemma will be quite useful when studying horoball diagrams on the surface of $B_{\infty}$ :

Lemma 3.4. Let $A, B$ be two horoballs not equal to $B_{\infty}$ and let $p, q$ be their orthocenters. Suppose $A \in \mathcal{O}(m)$ and $B \in \mathcal{O}(n)$, and suppose that $d(A, B)=O(r)$. Then the distance between $p$ and $q$ along the surface of $B_{\infty}$ is given by $d_{E}(p, q)=$ $e_{r} /\left(e_{m} e_{n}\right)$.

Proof. Using the upper half-space model, we may assume that $p=(0,0,1)$ and $q=(x, 0,1)$, where $x=d_{E}(p, q)$. Then $A$ is a ball of height $e_{m}^{-2}$ tangent to $S_{\infty}^{2}$ at 0 , and $B$ is a ball of height $e_{n}^{-2}$ tangent to $S_{\infty}^{2}$ at $(x, 0)$. Consider the following isometry given as an element of $\operatorname{PSL}(2, \mathbb{C})$ :

$$
\sigma=\left[\begin{array}{cc}
1 & 0 \\
-x^{-1} & 1
\end{array}\right]
$$

Since $\sigma$ is a parabolic isometry which fixes $0, \sigma$ preserves $A$. Also, $\sigma$ sends $x$ to $\infty$; therefore $\sigma$ sends $B$ to a horoball centered at infinity. The height of $\sigma(B)$ will be the same as the height of the image of the point $\left(x, 0, e_{n}{ }^{-2}\right)$, which is readily determined to be $x^{2} e_{n}{ }^{2}$. Therefore the distance between $A=\sigma(A)$ and $\sigma(B)$ is the logarithm of the ratio of $x^{2} e_{n}{ }^{2}$ to the diameter of $A$, which implies that

$$
e_{r}^{2}=\frac{x^{2} e_{n}^{2}}{e_{m}-2}
$$

which proves the lemma. 


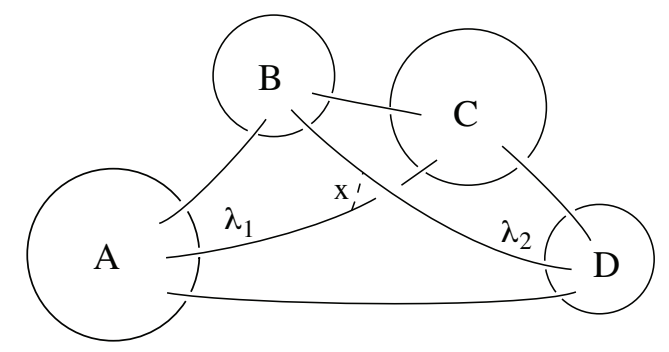

Figure 2. The horoballs and perpendiculars referred to in Lemma 3.5.

The next two lemmas concern pairs of lines joining the centers of horoballs in $\mathbb{H}^{3}$; they will be used extensively in discussing when geometric Mom- $n$ 's are embedded in Section 6.

Lemma 3.5. Suppose $A, B, C$, and $D$ are disjoint horoballs in $\mathbb{H}^{3}$ with pairwise disjoint interiors. Let $\lambda_{1}$ be the line joining the centers of $A$ and $C, \lambda_{2}$ be the line joining the centers of $B$ and $D$, and let $x$ be the distance between $\lambda_{1}$ and $\lambda_{2}$, as shown in Figure 2. Then

$$
e^{\frac{d(A, B)+d(C, D)}{2}}+e^{\frac{d(A, D)+d(B, C)}{2}}=e^{\frac{d(A, C)+d(B, D)}{2}} \cosh x .
$$

Corollary 3.6. If $\{A, B\} \in \mathcal{O}(h),\{B, C\} \in \mathcal{O}(j),\{C, D\} \in \mathcal{O}(k),\{D, A\} \in \mathcal{O}(l)$, $\{A, C\} \in \mathcal{O}(m)$, and $\{B, D\} \in \mathcal{O}(n)$, then

$$
e_{h} e_{k}+e_{j} e_{l}=e_{m} e_{n} \cosh x .
$$

Proof. Arrange the horoballs in the upper half-space model in such a way that $A$ is the horoball at infinity with height 1 and $C$ is centered at 0 . Let $z, w \in \mathbb{C}$ be the centers of $B$ and $D$, respectively. To compute the distance between the two lines $\lambda_{1}$ and $\lambda_{2}$, we turn to [Fen, which says that

$$
\cosh (x+i y)=-\frac{1}{2} \operatorname{tr}\left(\Lambda_{1} \Lambda_{2}\right),
$$

where

$$
\Lambda_{1}=\left[\begin{array}{cc}
i & 0 \\
0 & -i
\end{array}\right] \in \operatorname{PSL}(2, \mathbb{C})
$$

is an elliptic element of order 2 fixing the line from 0 to $\infty$ while

$$
\Lambda_{2}=\frac{i}{w-z}\left[\begin{array}{cc}
w+z & -2 w z \\
2 & -w-z
\end{array}\right] \in \operatorname{PSL}(2, \mathbb{C})
$$

is an elliptic element of order 2 fixing the line from $z$ to $w$, and where $y$ is the relative angle between the two lines along the shortest arc between them, which is 
only defined modulo $\pi$. Then by direct calculation,

$$
\begin{aligned}
\cosh (x+i y) & =-\frac{i}{2(w-z)} \operatorname{tr}\left(\left[\begin{array}{cc}
i & 0 \\
0 & -i
\end{array}\right]\left[\begin{array}{cc}
w+z & -2 w z \\
2 & -w-z
\end{array}\right]\right) \\
& =-\frac{i}{2(w-z)}(i(w+z)-i(-w-z)) \\
& =\frac{w+z}{w-z} .
\end{aligned}
$$

To determine $\cosh x$, note that as $y$ varies, $\cosh (x+i y)=\cosh x \cos y+i \sinh x \sin y$ varies along an ellipse in the complex plane. It is straightforward to determine that the foci of this ellipse are at -1 and 1 , and that therefore for any $y$,

$$
|\cosh (x+i y)-1|+|\cosh (x+i y)+1|=2 \cosh x .
$$

Therefore,

$$
\begin{aligned}
\cosh x & =\frac{1}{2}\left(\left|\frac{w+z}{w-z}-1\right|+\left|\frac{w+z}{w-z}+1\right|\right) \\
& =\frac{|w|+|z|}{|w-z|} .
\end{aligned}
$$

So $|w|+|z|=|w-z| \cosh x$. But by Lemma 3.4. $|w|^{2}=e^{d(C, D)-d(A, C)-d(A, D)}$, $|z|^{2}=e^{d(C, B)-d(A, C)-d(A, B)}$, and $|w-z|^{2}=e^{d(B, D)-d(A, B)-d(A, D)}$. The result follows.

Lemma 3.7. Let $N$ be a cusped hyperbolic manifold with cusp neighborhood $T$, and suppose $A, B, C$, and $D$ are all horoballs in $\mathbb{H}^{3}$ which are lifts of $T$. Define $\lambda_{1}$, $\lambda_{2}$, and $x$ as in the previous lemma. If there exists an element $g \in \pi_{1}(N)$ such that $g(A)=B$ and $g(C)=D$, and if $d(A, C)=d(B, D)$ is less than or equal to $2 \log (1.5152)$, then $x \geq 0.15$.

Proof. Suppose $x<0.15$; we will establish a contradiction. For the sake of notation, suppose that $\{A, C\}$ and $\{B, D\}$ are both elements of $\mathcal{O}(k)$. Note that this implies that $e_{k} \leq 1.5152$. Arrange the four horoballs in the upper half-space model so that $A$ is centered at infinity with height $e_{k}$ and $C$ is centered at 0 with height $e_{k}^{-1}$. Then suppose that

$$
g=\left[\begin{array}{ll}
a & b \\
c & d
\end{array}\right] \in \operatorname{PSL}(2, \mathbb{C}) .
$$

We wish to compute $x$ in terms of $a, b, c$, and $d$ in the same fashion as in the previous lemma. Since $g$ sends 0 to $\frac{b}{d}$ and $\infty$ to $\frac{a}{c}$, by the same arguments as before we have that for some $y$,

$$
\begin{aligned}
\cosh (x+i y) & =\frac{\frac{a}{c}+\frac{b}{d}}{\frac{a}{c}-\frac{b}{d}} \\
& =\frac{a d+b c}{a d-b c} \\
& =a d+b c \\
& =2 a d-1,
\end{aligned}
$$

where the last two steps used $a d-b c=1$. Hence

$$
a d=\frac{1}{2}(\cosh (x+i y)+1) .
$$




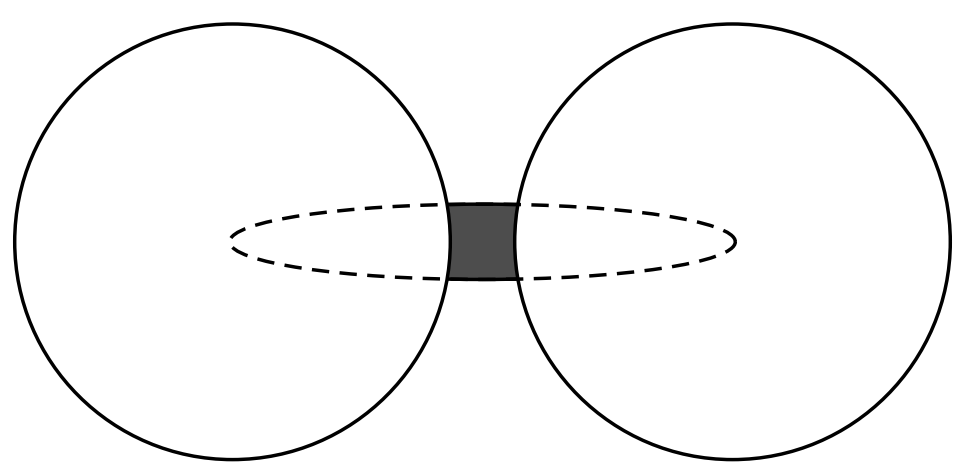

Figure 3 . The possible range of $a d$ in the complex plane. The shaded region is defined by the equation $|z-0|+|z-1| \leq \cosh 0.15$ (i.e., the solid ellipse bounded by the curve $\frac{1}{2}(\cosh (0.15+i t)+1)$, where $t$ is real) and the equations $|z| \geq 1.5152^{-2}$ and $|z-1| \geq$ $1.5152^{-2}$.

Since $x<0.15$, this implies that ad lies strictly inside an ellipse in the complex plane with foci at 0 and 1 , whose boundary intersects the real axis at the points $\frac{1}{2}(\cosh 0.15+1)$ and $\frac{1}{2}(1-\cosh 0.15)$.

Now recall that if $c \neq 0$, then the element $g$ sends a horoball of height $t$ centered at infinity to a horoball of height $t^{-1}|c|^{-2}$ (whereas if $c=0$, then $g$ fixes the point at infinity). So since $g(A)=B$ and since $B$ and $A$ have disjoint interiors, we must have $e_{k}^{-1}|c|^{-2} \leq e_{k}$, or in other words, $|c| \geq e_{k}^{-1} \geq 1.5152^{-1}$. But $C$ and $g(A)=B$ also have disjoint interiors, so let

$$
h=\left[\begin{array}{ll}
0 & i \\
i & 0
\end{array}\right] \in \operatorname{PSL}(2, \mathbb{C}) .
$$

Then $h$ swaps $A$ and $C$; therefore $h g(A)$ and $h(C)=A$ have disjoint interiors. Since

$$
h g=\left[\begin{array}{cc}
i c & i d \\
i a & i b
\end{array}\right]
$$

this implies that $e_{k}^{-1}|i a|^{-2} \leq e_{k}$, or in other words $|a| \geq e_{k}^{-1} \geq 1.5152^{-1}$. Similarly, $A$ and $D=g(C)=g h(A)$ have disjoint interiors, which implies that $|d| \geq 1.5152^{-1}$, and $C$ and $D$ have disjoint interiors, so $h(C)=A$ and $h(D)=h g h(A)$ have disjoint interiors, which implies that $|b| \geq 1.5152^{-1}$. Combining these facts we have $|a d| \geq 1.5152^{-2}$ and $|a d-1|=|b c| \geq 1.5152^{-2}$. Hence we can conclude that ad lies somewhere in the shaded region indicated in Figure 3 .

It's worthwhile at this point to sketch the motivation for the argument that follows. Roughly speaking, we've shown that ad is approximately equal to $1 / 2$. Given the stated lower bounds on $|a|$ and $|d|$, this implies that $|a|$ and $|d|$ are each approximately equal to $\sqrt{1 / 2}$. If it were the case that $a d=1 / 2$ and $|a|=$ $|d|=\sqrt{1 / 2}$, then $a+d$ would have to be a real number between $-\sqrt{2}$ and $\sqrt{2}$ which would imply that $g \in \pi_{1}(N)$ is elliptic, a contradiction. Since we only have approximate equality in the previous statement, we wish to show that $g$ is 
"approximately elliptic". More rigorously, we wish to show that $a+d$ lies close enough to the real interval from $-\sqrt{2}$ to $\sqrt{2}$ to ensure that $g^{n}(A)$ or $g^{n}(C)$ intersects $A$ for some $n$ (specifically $n=2,3$, or 4 ), a contradiction which will complete the proof of the lemma.

To make this argument work we need to break the problem into two cases depending on the value of $a d$. The first case will be when $\Re(a d) \geq 1 / 2$ and $a d$ lies in the shaded region in Figure 3; the second case is when $\Re(a d) \leq 1 / 2$. We proceed with the proof in the first case.

So suppose $\Re(a d) \geq 1 / 2$ and $a d$ lies in the shaded region in Figure 3; we wish to determine a domain for the trace $\tau=a+d$ of $g$. Suppose that $a=|a| e^{i \theta}$ and $d=|d| e^{i \phi} ;$ then

$$
\tau=e^{i\left(\frac{\theta+\phi}{2}\right)}\left((|a|+|d|) \cos \frac{\theta-\phi}{2}+i(|a|-|d|) \sin \frac{\theta-\phi}{2}\right) .
$$

Hence $\tau$ lies in an ellipse, centered at the origin, whose major axis has length $2(|a|+|d|)$ and whose minor axis has length 2||$a|-| d||$, and whose major axis is tilted away from the real axis by half the argument of $a d$.

Note that we have upper bounds on all three of these quantities. First, the argument of $a d$ is bounded since $a d$ is contained in a bounded simply connected region which does not contain 0 . Second, since $|a d|$ is bounded above and $|a|$ and $|d|$ are both bounded below, the point $(|a|,|d|)$ is contained in a region in the first quadrant of the real plane which is bounded by the lines $x=1.5152^{-1}$ and $y=1.5152^{-1}$ and the hyperbola $x y=D$, where $D$ is the maximum possible value of $|a d|$. This implies that $|a|+|d|$ and ||$a|-| d||$ are both bounded as well. Specifically, we can state the following:

$$
\begin{aligned}
\frac{\theta+\phi}{2} & \leq 0.07473, \\
|a|+|d| & \leq 1.5323, \\
|| a|-| d|| & \leq 0.2124 .
\end{aligned}
$$

These facts together imply that $\tau$ must be contained in a bounded region near the origin, as sketched in Figure 4 .

Now consider $g^{2}, g^{3}$, and $g^{4}$; these elements do not fix $\infty$ since $g$ does not. Similarly they do not fix 0 . By direct calculation,

$$
\begin{aligned}
g^{2} & =\left[\begin{array}{cc}
* & b \tau \\
c \tau & *
\end{array}\right], \\
g^{3} & =\left[\begin{array}{cc}
* & b\left(\tau^{2}-1\right) \\
c\left(\tau^{2}-1\right) & *
\end{array}\right], \\
g^{4} & =\left[\begin{array}{cc}
* & b\left(\tau^{3}-2 \tau\right) \\
c\left(\tau^{3}-2 \tau\right) & *
\end{array}\right] .
\end{aligned}
$$

(Here $*$ is used to denote entries whose value is unimportant.) Since $A$ and $g^{n}(A)$ (respectively $C$ and $g^{n}(C)$ ) have disjoint interiors, by the same arguments as before, all three of the quantities $|c \tau|,\left|c\left(\tau^{2}-1\right)\right|$, and $\left|c\left(\tau^{3}-2 \tau\right)\right|$ (respectively $|b \tau|, \mid b\left(\tau^{2}-\right.$ $1) \mid$, and $\left.\left|b\left(\tau^{3}-2 \tau\right)\right|\right)$ must be no less than $1.5152^{-1}$. Since $|b c|=|a d-1|$ and since $a d$ lies in a bounded simply connected region which does not contain $1, \sqrt{|b c|}$ is bounded above and hence $|\tau|,\left|\tau^{2}-1\right|$, and $\left|\tau^{3}-2 \tau\right|$ are bounded below. Specifically 


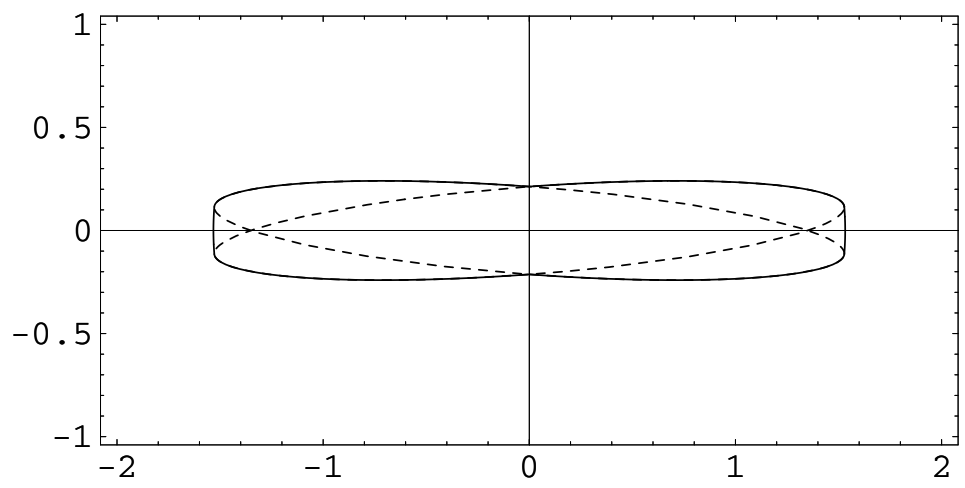

Figure 4. The region of possible values for $a+d$ (solid contour), generated by sweeping out ellipses with constant major axis and minor axis lengths, parameterized by the angle of inclination with the $x$-axis. The dashed contours represent the beginning and ending ellipses.

we have

$$
\begin{aligned}
|\tau| & \geq 0.9281 \\
\left|\tau^{2}-1\right| & \geq 0.9281 \\
\left|\tau^{3}-2 \tau\right| & \geq 0.9281
\end{aligned}
$$

But as demonstrated in Figure [5, the intersection of the three regions described by the above inequalities is disjoint from the previously determined range of possible values of $\tau$, a contradiction. This completes the proof in the first case.

The proof in the second case is nearly identical; only the specific numbers differ: replace 0.07473 with $0.08599,1.5323$ with $1.4262,0.2124$ with 0.1062 , and 0.9281 with 0.8698 . This completes the proof of the lemma.

The remaining lemmas in this chapter will be quite arbitrary and geometrical, but they will be quite useful in the next chapter when we begin to compute the volume of manifolds which do not possess geometric Mom- $n$ 's. We start with a lemma and definition, which first appeared in [CM]:

Lemma 3.8. Let $B$ be a horoball centered at infinity in the upper half-space model of $\mathbb{H}^{3}$, whose boundary has Euclidean height $1 / b$. Let $A$ be a half-space, i.e. one of the two connected components of the complement of a plane; assume that $A$ does not contain the point at infinity, and that the plane which defines $A$ appears in the upper half-space model as a Euclidean hemisphere with radius $1 / a$. Assume that $a<b$, so that $A \cap B$ is non-empty. Then the volume of $A \cap B$ is

$$
\pi\left(\frac{b^{2}}{2 a^{2}}-\frac{1}{2}+\log \frac{a}{b}\right) .
$$




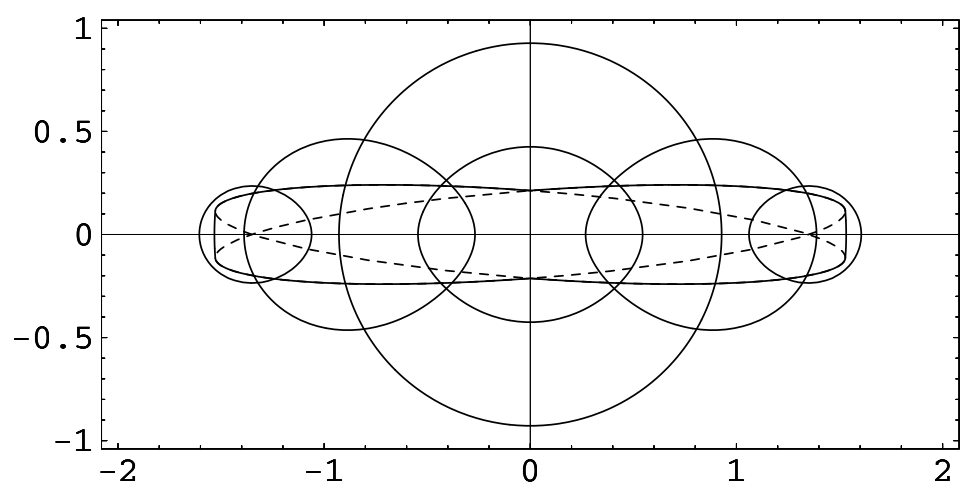

Figure 5. The region from Figure 4, contained inside the union of the regions $|\tau|<0.9281,\left|\tau^{2}-1\right|<0.9281$, and $\left|\tau^{3}-2 \tau\right|<0.9281$.

Definition 3.9. Denote the above quantity by lessvol $(a, b)$.

Proof of Lemma 3.8. Without loss of generality, assume that the plane defining $A$ appears as a Euclidean hemisphere centered at the origin. Then convert to cylindrical coordinates; the desired volume is given by the following integral:

$$
\int_{\frac{1}{b}}^{\frac{1}{a}} \int_{0}^{2 \pi} \int_{0}^{\sqrt{\frac{1}{a^{2}}-z^{2}}} \frac{r}{z^{3}} d r d \theta d z .
$$

The result follows immediately.

The following lemma is a straightforward exercise in trigonometry; the proof is left to the reader:

Lemma 3.10. Let $A$ and $B$ be two circular disks in the plane with radii $a$ and $b$, respectively, such that the centers of $A$ and $B$ are $c$ units apart. Suppose that $|a-b| \leq c \leq a+b$; i.e., suppose that the two disks overlap but neither disk is entirely contained in the interior of the other. Then

$$
\text { Area }(A \cap B)=a^{2} f\left(\frac{a^{2}-b^{2}+c^{2}}{2 a c}\right)+b^{2} f\left(\frac{-a^{2}+b^{2}+c^{2}}{2 b c}\right),
$$

where $f(x)=\cos ^{-1}(x)-x \sqrt{1-x^{2}}$ if $a \neq b$. If $a=b$, then

$$
\text { Area }(A \cap B)=2 a^{2} f\left(\frac{c}{2 a}\right) \text {. }
$$

Definition 3.11. Define the function overlapArea $(a, b, c)$ to equal the right-hand side of the first equation above if $a \neq b$, and the right-hand side of the second equation above if $a=b$.

Finally, we provide a companion to the above lemma. The function $f(x)$ described above, while relatively simple, turns out to be unsuitable for rigorous 
floating-pointing computation. As will be explained in more detail in Section 5, a polynomial approximation to $f(x)$ will be extremely useful. Hence we provide the following:

Lemma 3.12. Let $A$ and $B$ be two circular disks in the plane with radii a and $b$, respectively, such that the centers of $A$ and $B$ are $c$ units apart. Suppose that $|a-b| \leq c \leq a+b$; i.e., suppose that the two disks overlap but neither disk is entirely contained in the interior of the other. Then

$$
\text { Area }(A \cap B) \leq a^{2} g\left(\frac{a^{2}-b^{2}+c^{2}}{2 a c}\right)+b^{2} g\left(\frac{-a^{2}+b^{2}+c^{2}}{2 b c}\right),
$$

where $g(x)=\left(\frac{5}{3}-\frac{\pi}{2}\right) x^{5}+\frac{1}{3} x^{3}-2 x+\frac{\pi}{2}$ if $a \neq b$. If $a=b$, then

$$
\text { Area }(A \cap B) \leq 2 a^{2} g\left(\frac{c}{2 a}\right) \text {. }
$$

Definition 3.13. Define the function overlapApprox $(a, b, c)$ to equal the right-hand side of the first inequality above if $a \neq b$, and the right-hand side of the second inequality above if $a=b$.

Proof. Comparing the above lemma to Lemma 3.10, clearly it would suffice to show that $g(x) \geq f(x)$ for all $x \in[-1,1]$, where $f(x)=\cos ^{-1}(x)-x \sqrt{1-x^{2}}$. Unfortunately, this cannot be the case as both $f(x)-\frac{\pi}{2}$ and $g(x)-\frac{\pi}{2}$ are odd functions. However, it is true that $g(x) \geq f(x)$ for all $x \in[0,1]$; we will use this fact in what follows. Let $h(x)=g(x)-f(x)$. We note the following facts about $h(x)$, which are readily verified: $h(x)$ is an odd function, $h( \pm 1)=h(0)=0, h(x)>0$ if $0<x<1$, and $h(x)$ has a single local maximum at $x_{\max }=0.895 \ldots$ By Lemma 3.10 it suffices to show that

$$
a^{2} h\left(\frac{a^{2}-b^{2}+c^{2}}{2 a c}\right)+b^{2} h\left(\frac{-a^{2}+b^{2}+c^{2}}{2 b c}\right) \geq 0
$$

if $a \neq b$.

Let $P$ be the center of the first circle, $Q$ the center of the second, and let $R$ be one of the points where the circles intersect. Let $\theta$ be the angle of the triangle $P Q R$ at $P$, and let $\phi$ be the angle at $Q$. Applying the law of cosines for $P Q R$ to the above inequality, we get

$$
a^{2} h(\cos \theta)+b^{2} h(\cos \phi) \geq 0 .
$$

Note that if $\theta=0$ or $\pi$, then $\phi=0$ or $\pi$ and vice versa. Since $h(1)=h(-1)=0$ the lemma is true in either case. So suppose that $\theta, \phi \in(0, \pi)$. Note that since $\theta$ and $\phi$ are angles of a (possibly flat) triangle, this also implies that $\theta+\phi \in(0, \pi)$. Then we can apply the law of sines to the above inequality to get

$$
\left(\frac{c \sin \phi}{\sin (\theta+\phi)}\right)^{2} h(\cos \theta)+\left(\frac{c \sin \theta}{\sin (\theta+\phi)}\right)^{2} h(\cos \phi) \geq 0 .
$$

Letting $x=\cos \theta$ and $y=\cos \phi$, it suffices to show that

$$
\left(1-y^{2}\right) h(x)+\left(1-x^{2}\right) h(y) \geq 0,
$$

where $x<1, y<1$, and $x>-y$ (since $\theta<\pi-\phi$ ). Let $H(x, y)$ equal the left-hand side of the above inequality. Note that $H(x, 1)=H(1, y)=0$ since $h(1)=0$, and $H(x,-x)=0$ for $x \in[-1,1]$ since $h(x)$ is odd. Hence the lemma will be proved if we can show that $H(x, y)$ has a non-negative value at any local minimum in the 
interior of the triangle bounded by the lines $x=1, y=1$, and $x+y=0$. Since $h(x) \geq 0$ if $0 \leq x \leq 1, H(x, y) \geq 0$ whenever $0 \leq x \leq 1$ and $0 \leq y \leq 1$. Also, $H(x, y)=H(y, x)$. Therefore it suffices to examine local minima in the interior of the triangle bounded by the lines $x=1, y=0$, and $x+y=0$. We now show that there are no such local minima in that region.

Computing the gradient, we see that if $\left(x_{0}, y_{0}\right)$ is a local extreme point of $H(x, y)$, then

$$
\begin{aligned}
& \left(1-y_{0}^{2}\right) h^{\prime}\left(x_{0}\right)=2 x_{0} h\left(y_{0}\right), \\
& \left(1-x_{0}^{2}\right) h^{\prime}\left(y_{0}\right)=2 y_{0} h\left(x_{0}\right) .
\end{aligned}
$$

If $\left(x_{0}, y_{0}\right)$ is in the interior of the triangle described above, then $0<x_{0}<1$ and $-1<y_{0}<0$. Then from the first equation, since $h\left(y_{0}\right)<0$, we must have $h^{\prime}\left(x_{0}\right)<0$, and from the second equation, since $h\left(x_{0}\right)>0$, we must have $h^{\prime}\left(y_{0}\right)<0$. Therefore $x_{\max }<x_{0}<1$ and $-1<y_{0}<-x_{\max }$. Now suppose further that $\left(x_{0}, y_{0}\right)$ is a local minimum. Then we must have $\partial^{2} H / \partial x^{2}>0$ at that point, which implies that

$$
h^{\prime \prime}\left(x_{0}\right)>\frac{2 h\left(y_{0}\right)}{1-y_{0}^{2}} .
$$

But it can be readily computed that the maximum value of $h^{\prime \prime}(x)$ on the interval $\left[x_{\max }, 1\right]$ is less than -0.85 , while the minimum value of $2 h\left(y_{0}\right) /\left(1-y_{0}^{2}\right)$ on the interval $\left[-1,-x_{\max }\right]$ is greater than -0.6 , contradicting the above inequality. This completes the proof of the lemma.

\section{Bounding the Parameter SPaCe}

In Section 5 we will show that a one-cusped hyperbolic 3-manifold with volume less than or equal to 2.848 has a geometric Mom-2 or Mom-3 structure. This proof involves constructing volume estimates in terms of the first three non-trivial elements of the Euclidean spectrum $e_{2}, e_{3}$, and $e_{4}$, and then evaluating those formulas with rigorous computer assistance. This requires us to restrict our attention from the space of all possible values of $\left(e_{2}, e_{3}, e_{4}\right)$ to a compact subset of that space. Doing so is the purpose of this section.

Specifically, we wish to prove the following:

Proposition 4.1. Let $N$ be a one-cusped hyperbolic 3-manifold with $\operatorname{Vol}(N) \leq$ 2.848. Then $e_{2} \leq 1.4751$; furthermore, one of the following statements must be true:

- $e_{3} \leq 1.5152$, or

- $N$ contains a geometric Mom-2 structure consisting only of $(1,1,2)$-triples and (1,2,2)-triples.

Note that this effectively provides upper bounds on $e_{2}$ and $e_{3}$ for manifolds with $\operatorname{Vol}(N) \leq 2.848$; lower bounds are trivially provided by $e_{k} \geq e_{1}=1$. This proposition does not provide an upper bound for $e_{4}$, but we can do without as described in the next section.

The proof of Proposition 4.1 depends on several complicated estimates of area and volume and spans multiple cases. Similar arguments, presented in less detail for brevity, will be used for the proofs in Section 5, and hence this section should perhaps be considered a "warm-up" for those results. 
We begin with the following simple result:

Lemma 4.2. The volume of a one-cusped hyperbolic three-manifold $N$ is at least

$$
\frac{e_{2}{ }^{4} \sqrt{3}}{2}-\pi\left(e_{2}^{2}-1-2 \log e_{2}\right) .
$$

Corollary 4.3. If $e_{2}>1.4751$, then $\operatorname{Vol}(N)>2.848$.

Proof. Let $\left\{p_{i}\right\} \subset \partial B_{\infty}$ be the set of orthocenters of horoballs belonging to $\mathcal{O}(1)$. According to Lemmas 3.2 and 3.4, $d_{E}\left(p_{i}, p_{j}\right) \geq e_{2}$ for all $i \neq j$. Therefore $\partial B_{\infty}$ can be packed by circles of radius $e_{2} / 2$ centered at each point $p_{i}$. There are two $H$-orbits of such points by Lemma 3.1, and therefore the area of $\partial B_{\infty} / H$ is at least $\pi\left(e_{2}{ }^{2}\right) / 2$. Since our packing is by circles of uniform radius, we can immediately improve this bound by a factor of $\sqrt{12} / \pi$ (the density of the hexagonal circle packing) to get

$$
\operatorname{Area}\left(\partial B_{\infty} / H\right) \geq e_{2}^{2} \sqrt{3} \text {. }
$$

However we want to estimate volume, not area. The volume of $B_{\infty} / H$ is exactly Area $\left(\partial B_{\infty} / H\right) / 2$, and this is a lower bound on the volume of $N$, but this lower bound only accounts for the volume inside the cusp neighborhood. We would like our estimate to count some of the volume outside the cusp neighborhood as well.

We do this by "inflating" the cusp neighborhood in a uniform fashion. In the upper half-space model, this corresponds to replacing $B_{\infty}$ with a new horoball $C$ which is centered at infinity but whose Euclidean height is some positive number which may be less than one. In particular we choose $C$ to have height $1 / e_{2}$. Then

$$
\begin{aligned}
\operatorname{Vol}(C / H) & =\frac{e_{2}{ }^{2}}{2} \operatorname{Area}\left(\partial B_{\infty} / H\right) \\
& =\frac{e_{2}{ }^{4}}{2} \sqrt{3},
\end{aligned}
$$

but of course $C / H$ is no longer embedded in $N$; it is only immersed. To get a valid lower bound for $\operatorname{Vol}(N)$ we must "cut off" $C / H$ by subtracting the volumes of those regions where $C / H$ extends beyond the Ford domain corresponding to $B_{\infty} / H$. The maximum height of a Ford face corresponding to $A \in \mathcal{O}(2)$ is just $1 / e_{2}$; therefore $C / H$ only extends past the Ford faces corresponding to $\mathcal{O}(1)$-horoballs and not $\mathcal{O}(2)$-horoballs (or $\mathcal{O}(n)$-horoballs for $n>2$ ). Hence at most we must subtract twice the volume of the region where the horoball $C$ intersects the halfspace corresponding to some $B \in \mathcal{O}(1)$. That volume is given by the lessvol function (see Definition 3.9); specifically,

$$
\begin{aligned}
\operatorname{Vol}(N) & \geq \operatorname{Vol}(C / H)-2 \operatorname{lessvol}\left(1, e_{2}\right) \\
& =\frac{e_{2}{ }^{4} \sqrt{3}}{2}-\pi\left(e_{2}{ }^{2}-1-2 \log e_{2}\right) .
\end{aligned}
$$

This is the desired result.

The corollary follows since the above function is easily verified to be increasing in $e_{2}$.

In practice, the area of the cusp torus $\partial B_{\infty} / H$ will usually be far greater than our crude estimate of $e_{2}^{2} \sqrt{3}$, because the density of the packing of $\partial B_{\infty}$ by circles around the orthocenters of the $\mathcal{O}(1)$-horoballs is typically nowhere near optimal. One strategy to try and capture the extra area between the circles is simply to use larger circles: specifically, use circles of radius $e_{3} / 2$ instead of $e_{2} / 2$ to estimate the 
area. This has the advantage of incorporating $e_{3}$ into the area estimate, which will lead to the upper bound on $e_{3}$ that we seek. The disadvantage, of course, is that by increasing the radius we can no longer guarantee that each circle will be embedded in $\partial B_{\infty} / H$ : the larger circles may overlap.

However, such overlaps imply the existence of triples which may be part of a geometric Mom- $n$ structure. If two circles of radius $e_{3} / 2$ overlap, then the distance between their centers is less than $e_{3}$, which implies by Lemma 3.4 that the corresponding horoballs, together with $B_{\infty}$, must form a $(1,1,2)$-triple. If there are no such triples, then our new expanded circles will not overlap. This leads to the following:

Lemma 4.4. Let $N$ be a one-cusped hyperbolic three-manifold whose horoball diagram contains no triples of type $(1,1,2)$. Then

$$
\operatorname{Vol}(N) \geq \frac{e_{2}^{2} e_{3}^{2} \sqrt{3}}{2}-\pi\left(e_{2}^{2}-1-2 \log e_{2}\right)
$$

Corollary 4.5. Under the assumptions of the lemma, if $e_{3}>1.8135$, then $\operatorname{Vol}(N)$ $>2.848$.

Proof. Let $\left\{p_{i}\right\} \subset \partial B_{\infty}$ be defined as in the proof of Theorem 4.2. Since the horoball diagram for $N$ contains no $(1,1,2)$ handles, $d_{E}\left(p_{i}, p_{j}\right) \geq e_{3}$ for all $i \neq j$, and $\partial B_{\infty}$ can be packed by circles of radius $e_{3} / 2$ centered at each point $p_{i}$. The rest of the proof proceeds just as in the proof of Theorem 4.2 .

To prove the corollary, note that the right-hand side of equation (11) is both increasing in $e_{2}$ for fixed $e_{3}$ and vice versa. When $e_{3}=1.8135$ and $e_{2}=1, \operatorname{Vol}(N)>$ 2.848 and the result follows.

The upper bound on $e_{3}$ obtained from Corollary 4.5 is unfortunately too large to be useful. The next step is to improve upon it by accounting for the effect of the horoballs in $\mathcal{O}(2)$ upon Area $\left(\partial B_{\infty}\right)$. If $e_{2}$ is small, then we expect to be able to construct circles around the orthocenters of these horoballs which will be almost as large as the circles around the orthocenters of the horoballs in $\mathcal{O}(1)$, increasing our estimate. While if $e_{2}$ is large, then $e_{3}$ must be large as well, increasing the area of the circles constructed in the proof of Lemma 4.4 .

Thus we wish to estimate $\operatorname{Area}\left(\partial B_{\infty}\right)$ using two sets of circles: one set corresponding to the elements of $\mathcal{O}(1)$ as before, and another set corresponding to the elements of $\mathcal{O}(2)$. But we need to decide how large the circles in this new second set will be. Also, whatever new circles we construct may intersect the previously constructed circles corresponding to the $\mathcal{O}(1)$ horoballs. In particular, if $N$ possesses a $(1,2,2)$-triple, then $\mathcal{O}(2)$-horoballs and $\mathcal{O}(1)$-horoballs will be close enough for these circles to possibly intersect. This can be accounted for using the function overlapArea $(a, b, c)$ from Lemma 3.10, leading to the following:

Lemma 4.6. Let $N$ be a one-cusped hyperbolic 3-manifold containing no triples of type $(1,1,2)$ and at most one triple of type $(1,2,2)$. Furthermore, assume that 
$e_{2} \leq 1.4751$, and $e_{3} \leq 1.8135$. Then

$$
\begin{aligned}
\operatorname{Area}\left(\partial B_{\infty} / H\right) \geq & 2 \pi\left(\frac{e_{3}}{2}\right)^{2}+2 \pi\left(\frac{e_{3}}{e_{2}}-\frac{e_{3}}{2}\right)^{2} \\
& -2 \text { overlapArea }\left(\frac{e_{3}}{e_{2}}-\frac{e_{3}}{2}, \frac{e_{3}}{2}, 1\right) \\
& - \text { overlapArea }\left(\frac{e_{3}}{e_{2}}-\frac{e_{3}}{2}, \frac{e_{3}}{e_{2}}-\frac{e_{3}}{2}, \frac{1}{e_{2}{ }^{2}}\right)
\end{aligned}
$$

and furthermore

$$
\operatorname{Vol}(N) \geq \operatorname{Area}\left(\partial B_{\infty} / H\right) \frac{e_{3}^{2}}{2}-\pi\left(e_{3}^{2}-1-2 \log e_{3}+\frac{e_{3}^{2}}{e_{2}{ }^{2}}-1-2 \log \frac{e_{3}}{e_{2}}\right) .
$$

Corollary 4.7. Under the assumptions of Lemma 4.6 if $e_{3}>1.4751$ then $\operatorname{Vol}(N)>$ 2.848 .

Proof. Let $\left\{p_{i}\right\}$ be the set of orthocenters of $\mathcal{O}(1)$ horoballs as before, and let $\left\{q_{i}\right\}$ be the set of orthocenters of $\mathcal{O}(2)$ horoballs. Assume that we have already constructed circles of radius $e_{3} / 2$ around each $p_{i}$ as in Lemma 4.4. As before, since there is not a $(1,1,2)$-triple, these circles will not overlap.

Now in addition to this, construct circles of radius $e_{3} / e_{2}-e_{3} / 2$ around each of the points $q_{i}$. These circles may conceivably overlap either the previous circles or each other. Suppose that the circle around $q_{i}$ overlaps the circle around $p_{j}$ for some $i$ and $j$, and let $B_{i}$ and $B_{j}$ be the corresponding horoballs. Then clearly $d_{E}\left(q_{i}, p_{j}\right)<e_{3} / e_{2}$, which by Lemma 3.4 implies that $d\left(B_{i}, B_{j}\right)<o(3)$. Therefore $\left(B_{i}, B_{j}, B_{\infty}\right)$ must be a triple of type $(1,2,2)$ (or a triple of type $(1,1,2)$, but we're explicitly excluding that case right now).

Or suppose that the circles around $q_{i}$ and $q_{j}$ overlap for some $i$ and $j$, and let $B_{i}$ and $B_{j}$ be the corresponding horoballs. If $d\left(B_{i}, B_{j}\right) \geq o(3)$, then by Lemma 3.4 we must have $d_{E}\left(q_{i}, q_{j}\right) \geq e_{3} / e_{2}{ }^{2}$. But it is easy to show that $2\left(e_{3} / e_{2}-e_{3} / 2\right) \leq$ $e_{3} / e_{2}{ }^{2}$ for all positive $e_{2}$ and $e_{3}$; therefore if the circles are indeed overlapping, then $d\left(B_{i}, B_{j}\right)<o(3)$. Therefore $\left(B_{i}, B_{j}, B_{\infty}\right)$ must again be a triple of type $(1,2,2)$ (recall that a triple of type $(2,2,2)$ is impossible by Lemma 3.2).

In summary, any overlaps between the new circles and the old ones, or between the new circles and each other, arise due to the presence of a triple of type $(1,2,2)$. Also, we have supposed that there is no more than one such triple up to the action of $G$.

After taking the quotient by the action of $H$ we are left with two new circles of radius $e_{3} / e_{2}-e_{3} / 2$ and up to three new cases where one circle overlaps another. First, a $(1,2,2)$-triple implies that $d_{E}\left(q_{i}, q_{j}\right)=1 / e_{2}{ }^{2}$ for some $i$ and $j$; therefore in $\partial B_{\infty} / H$ we may see either the two new circles overlapping each other or else we may see one of the new circles overlapping itself. Secondly, a $(1,2,2)$-triple implies that $d_{E}\left(q_{i}, p_{j}\right)=1$ for some $i$ and $j$; in $\partial B_{\infty} / H$ we may see up to two instances of a new circle being overlapped by an old one.

Therefore the area of $\partial B_{\infty} / H$ is at least the area of the two new circles plus the two old circles minus the three possible overlaps. Using Lemma 3.10, this proves the first half of the lemma.

There is a technical issue that must be addressed: Lemma 3.10 is not valid if the circles in question do not overlap, or if one circle is contained in the interior of 
the other. Since we're using Lemma 3.10 twice we need to ensure that both of the following sets of inequalities hold:

$$
\begin{aligned}
\left|\frac{e_{3}}{e_{2}}-e_{3}\right| & \leq 1 \leq \frac{e_{3}}{e_{2}} \\
0 & \leq e_{2}{ }^{-2} \leq \frac{2 e_{3}}{e_{2}}-e_{3} .
\end{aligned}
$$

All of the above inequalities can be verified by elementary means when $1 \leq e_{2} \leq$ 1.4751 and $e_{2} \leq e_{3} \leq 1.8135$. Hence our use of Lemma 3.10 is valid. Note that this also confirms that the new circles actually contribute to the area of $\partial B_{\infty} / H$.

To find a lower bound of the volume of $N$, we inflate the cusp neighborhood as in the proof of Theorem 4.2. This time we obtain a horoball $C$ centered at infinity with Euclidean height $1 / e_{3}$. Then $\operatorname{Vol}(C / H)=\frac{1}{2} \operatorname{Area}\left(\partial B_{\infty} / H\right) e_{3}{ }^{2}$. Also, while $C / H$ extends beyond the Ford domain corresponding to $B_{\infty} / H$, at worst it only extends past the Ford faces corresponding to $\mathcal{O}(1)$-horoballs and $\mathcal{O}(2)$-horoballs. Hence,

$$
\operatorname{Vol}(N) \geq \operatorname{Area}\left(\partial B_{\infty} / H\right) \frac{e_{3}^{2}}{2}-2 \operatorname{lessvol}\left(1, e_{3}\right)-2 \operatorname{lessvol}\left(e_{2}, e_{3}\right)
$$

This proves the second half of the lemma. To prove the corollary, note that the resulting volume bound is increasing in $e_{3}$ for fixed $e_{2}$ and decreasing in $e_{2}$ for fixed $e_{3}$. Hence the minimum value of the volume bound over the domain $1 \leq e_{2} \leq$ $1.4751,1.4751 \leq e_{3} \leq 1.8135$ occurs when $e_{2}=e_{3}=1.4751$, and at the point $\operatorname{Vol}(N)>2.848$.

The next step is to perform the same analysis in the case where the horoball diagram for $N$ contains exactly one triple of type $(1,1,2)$ and no triples of type $(1,2,2)$. (Note that if $N$ had at least one of each type of triple, then $N$ would have a geometric Mom-2.) This analysis is similar to that of the previous case, and therefore in what follows some details are omitted.

Lemma 4.8. Let $N$ be a one-cusped hyperbolic 3-manifold whose horoball diagram contains one triple of type $(1,1,2)$. Then

$$
\operatorname{Area}\left(\partial B_{\infty} / H\right) \geq 2 \pi\left(\frac{e_{3}}{2}\right)^{2}-\text { overlapArea }\left(\frac{e_{3}}{2}, \frac{e_{3}}{2}, e_{2}\right)
$$

and furthermore

$$
\operatorname{Vol}(N) \geq \operatorname{Area}\left(\partial B_{\infty} / H\right) \frac{e_{2}^{2}}{2}-\pi\left(e_{2}^{2}-1-2 \log e_{2}\right) .
$$

Corollary 4.9. Under the same assumptions as in Lemma 4.8, if $e_{3}>2.1491$, then $\operatorname{Vol}(N)>2.848$.

Proof. As in the proof of Lemma 4.4, we wish to tile $\partial B_{\infty} / H$ with disks of radius $e_{3} / 2$ centered at the centers of the $\mathcal{O}(1)$-horoballs. But since in this case there is by assumption a single $(1,1,2)$-triple, such disks will overlap exactly once. This, together with Lemma 3.10 proves the first part of the lemma. (It is a trivial matter to confirm that 3.10 applies; the relevant inequality is $0 \leq e_{2} \leq e_{3}$.) To prove the second part, we inflate the cusp to obtain a horoball $C$ centered at infinity and with Euclidean height $1 / e_{2}$ and then proceed just as in Theorem 4.2 .

The resulting bound on $\operatorname{Vol}(N)$ is increasing in $e_{3}$ for fixed $e_{2}$ and vice versa, and when $e_{2}=1$ and $e_{3}=2.1491$ we get $\operatorname{Vol}(N)>2.848$; this proves the corollary. 
We now wish to improve the bound on $e_{3}$ by mimicking the argument used in the previous case. That is, we wish to construct additional circles in the horoball diagram of radius $e_{3} / e_{2}-e_{3} / 2$ corresponding to the $\mathcal{O}(2)$ horoballs as before. As in the previous case, such circles are small enough that the only overlaps between them and the circles of radius $e_{3} / 2$ will arise as a result of the presence of a $(1,1,2)$ triple (of which we assume there is at most one) or a $(1,2,2)$-triple (of which we will assume there are none at all).

Here we run into a problem, however, when we try to confirm that Lemma 3.10 applies, or in other words when we try to confirm that the new circles are both overlapped by the old ones and not completely contained within the old ones. According to Lemma 3.4, in the presence of a $(1,1,2)$ triple the orthocenter of an $\mathcal{O}(2)$ horoball will be at a distance of $1 / e_{2}$ from the center of some $\mathcal{O}(1)$ horoball. Therefore Lemma 3.10 applies if and only if the following inequalities hold:

$$
\left|\frac{e_{3}}{e_{2}}-e_{3}\right| \leq \frac{1}{e_{2}} \leq \frac{e_{3}}{e_{2}}
$$

The right-hand inequality is trivially true since $e_{3} \geq 1$ but the left-hand inequality simplifies to $e_{3} \leq\left(e_{2}-1\right)^{-1}$. This last inequality is not always true in the region $1 \leq e_{2} \leq 1.4751, e_{2} \leq e_{3} \leq 2.1491$. So we have to be a little more clever. Note that the inequality $e_{3} \leq\left(e_{2}-1\right)^{-1}$ fails when $e_{3}$ and $e_{2}$ are both large, but the worst case in the previous lemma occurred when $e_{2}$ was small. Hence a more sophisticated version of Corollary 4.9 is required, one which lets us restrict our attention to a smaller region which does not intersect the curve $e_{3}=\left(e_{2}-1\right)^{-1}$ :

Corollary 4.10. Under the assumptions of Lemma 4.8, if $e_{3}>2.1491-\left(e_{2}-1\right)$, then $\operatorname{Vol}(N)>2.848$.

Proof. This follows from two observations: first, that the volume estimate in Lemma 4.8 is increasing in $e_{3}$ for fixed $e_{2}$; second, if $e_{3}=2.1491-\left(e_{2}-1\right)$, then $\operatorname{Vol}(N)>$ 2.848 , as can be readily verified by direct computation.

Note that the curve $e_{3}=\left(e_{2}-1\right)^{-1}$ lies above the line $e_{3}=2.1491-\left(e_{2}-1\right)$. Thus with the preceding corollary in hand, we can now prove the following:

Lemma 4.11. Let $N$ be a one-cusped hyperbolic 3-manifold containing no triples of type $(1,2,2)$ and one triple of type $(1,1,2)$. Furthermore, assume that $e_{2} \leq 1.4751$ and $e_{3} \leq 2.1491-\left(e_{2}-1\right)$. Then

$$
\begin{aligned}
\operatorname{Area}\left(\partial B_{\infty} / H\right) \geq & 2 \pi\left(\frac{e_{3}}{2}\right)^{2}+2 \pi\left(\frac{e_{3}}{e_{2}}-\frac{e_{3}}{2}\right)^{2}-\text { overlapArea }\left(\frac{e_{3}}{2}, \frac{e_{3}}{2}, e_{2}\right) \\
& -2 \text { overlapArea }\left(\frac{e_{3}}{e_{2}}-\frac{e_{3}}{2}, \frac{e_{3}}{2}, \frac{1}{e_{2}}\right)
\end{aligned}
$$

and furthermore

$$
\operatorname{Vol}(N) \geq \operatorname{Area}\left(\partial B_{\infty} / H\right) \frac{e_{3}^{2}}{2}-\pi\left(e_{3}{ }^{2}-1-2 \log e_{3}+\frac{e_{3}{ }^{2}}{e_{2}{ }^{2}}-1-2 \log \frac{e_{3}}{e_{2}}\right) .
$$

Corollary 4.12. Under the assumptions of Lemma 4.11, if $e_{3}>1.5152$, then $\operatorname{Vol}(N)>2.848$. 
Proof. As promised, this proof will be somewhat light on details due to the extreme similarity to the previous case. Let $\left\{p_{i}\right\}$ be the orthocenters of the $\mathcal{O}(1)$ horoballs and let $\left\{q_{i}\right\}$ be the orthocenters of the $\mathcal{O}(2)$ horoballs as before. Construct circles of radius $e_{3} / 2$ around the $p_{i}$ 's. As in Lemma 4.8 there is one overlap between those two disks, caused by the $(1,1,2)$-triple. In addition, we also have circles of radius $e_{3} / e_{2}-e_{3} / 2$ centered at the $q_{i}$ 's. These circles do not intersect each other since there are by assumption no $(1,2,2)$-triples in $N$; however since there is one $(1,1,2)$ triple there will be two overlaps between these circles and the circles of radius $e_{3} / 2$. This together with Lemma 3.10 and our observations after Corollary 4.10 prove the first part of the lemma. To prove the second part, we inflate the cusp to obtain a horoball $C$ centered at infinity with Euclidean height $1 / e_{3}$, which at worst extends past the Ford faces corresponding to the $\mathcal{O}(1)$-horoballs and $\mathcal{O}(2)$-horoballs, then apply the lessvol function as before. The resulting bound on $\operatorname{Vol}(N)$ is increasing in $e_{3}$ for fixed $e_{2}$, and decreasing in $e_{2}$ for fixed $e_{3}$, and when $e_{2}=1.4751$ and $e_{3}=1.5152$ we get $\operatorname{Vol}(N)>2.848$. This proves the corollary.

We now finally have enough tools to prove the main result of this section:

Proof of Proposition 4.1. Suppose that $N$ is such that $\operatorname{Vol}(N) \leq 2.848$. Then $e_{2} \leq$ 1.4751 by Corollary 4.3 .

Suppose that $N$ does not contain a geometric Mom-2 of the type described in the proposition; we wish to show that this implies $e_{3} \leq 1.5152$. Our assumption implies that $N$ cannot contain:

- two or more $(1,1,2)$-triples,

- two or more $(1,2,2)$-triples,

- both a (1,1,2)-triple and a (1,2,2)-triple.

So if $N$ contains no $(1,1,2)$-triples, then it contains at most one $(1,2,2)$-triple and Corollaries 4.5 and 4.7 apply. If $N$ contains exactly one $(1,1,2)$-triple, then it must contain no $(1,2,2)$-triples and Corollaries 4.9 and 4.12 apply, completing the proof.

\section{Finding A GeOMetric Mom- $n, n=2$ or 3}

The goal of this section is to strengthen the results of the previous section to obtain the following:

Proposition 5.1. Let $N$ be a one-cusped hyperbolic 3-manifold with $\operatorname{Vol}(N) \leq$ 2.848. Then $N$ possesses a geometric Mom-n structure for $n=2$ or 3 which is torus-friendly.

The technique used to prove the above theorem is identical in principle to the techniques used to prove Proposition 4.1. Namely, we assume that $N$ does not possess such a geometric Mom- $n$ structure and then construct a lower bound on the area of the cusp torus. Specifically we will construct circles around the orthocenters of horoballs in $\mathcal{O}(1), \mathcal{O}(2)$, and $\mathcal{O}(3)$. The circles we will construct will be large enough that they will overlap one another, but we can use the lack of a Mom-2 or Mom-3 structure to carefully limit the number of such overlaps that will occur. Once we have a bound on the area of the cusp torus we can use that (along with a careful estimate of the volume of the manifold outside the cusp neighborhood) to find a bound on the volume of $N$. The difficulties, as we will see, are in the number 
of cases to be considered and the complex nature of the volume bounds that result. But for now, we begin with the following:

Definition 5.2. Let $e_{\max }=\min \left(e_{4}, 1.5152\right)$, and let

$$
A_{0}=\sum_{i=1}^{3} 2 \pi\left(e_{\max }\left(e_{i}{ }^{-1}-\frac{1}{2}\right)\right)^{2}
$$

Recall that by the results of the previous section we may assume that $e_{3} \leq$ $1.5152<2$; therefore $e_{\max }\left(e_{i}{ }^{-1}-1 / 2\right)$ is always positive for $i \leq 3$. Furthermore, if $p_{i}$ and $p_{j}$ are the orthocenters of horoballs $B_{i} \in \mathcal{O}(i)$ and $B_{j} \in \mathcal{O}(j)$, where $i, j \in\{1,2,3\}$, and if $d\left(B_{i}, B_{j}\right) \geq o(4)$, then $d_{E}\left(p_{i}, p_{j}\right) \geq e_{4} / e_{i} e_{j}$ by Lemma 3.4. Then we have the following:

$$
\begin{aligned}
\frac{e_{4}}{e_{i} e_{j}}-e_{\max }\left(e_{i}{ }^{-1}-\frac{1}{2}\right)-e_{\max }\left(e_{j}{ }^{-1}-\frac{1}{2}\right) & \geq \frac{e_{4}}{e_{i} e_{j}}\left(1-e_{i}\right)\left(1-e_{j}\right) \\
& \geq 0
\end{aligned}
$$

where the last line follows since $e_{i} \geq 1$ for all $i$.

Therefore if we place a circle of radius $e_{\max }\left(e_{i}{ }^{-1}-1 / 2\right)$ around the orthocenter of both of the horoballs in $\mathcal{O}(i)$, for $i \in\{1,2,3\}$, and if the manifold $N$ does not possess any horoball triples involving $\mathcal{O}(1), \mathcal{O}(2)$, and $\mathcal{O}(3)$, then those six circles will have disjoint interiors and therefore $A_{0}$ will be a lower bound for the area of the cusp torus $\partial B_{\infty} / H$. Of course it is highly unlikely that $N$ will possess no such triples, and thus we must consider the possibility that some of those six circles will overlap. This leads to our next definition:

Definition 5.3. Let

$$
l_{i, j, k}=\text { overlapApprox }\left(e_{\max }\left(e_{i}{ }^{-1}-\frac{1}{2}\right), e_{\max }\left(e_{j}{ }^{-1}-\frac{1}{2}\right), c\right),
$$

where $c=\min \left(e_{k} /\left(e_{i} e_{j}\right), e_{\max }\left(e_{i}^{-1}+e_{j}{ }^{-1}-1\right)\right)$.

Recall that overlapApprox $(a, b, c)$ was defined in Definition 3.13, and by Lemma 3.12 it is always greater than or equal to the area of the intersection of a circle of radius $a$ and a circle of radius $b$ whose centers are $c$ units apart, provided that $|a-b| \leq c \leq a+b$.

Hence if $p_{i}, p_{j}$ are respectively the orthocenters of horoballs $B_{i} \in \mathcal{O}(i), B_{j} \in$ $\mathcal{O}(j)$, and if $d\left(B_{i}, B_{j}\right)=o(k)$, where $i, j$, and $k$ are all in $\{1,2,3\}$, then $l_{i, j, k}$ will be greater than or equal to the amount of overlap between a circle of radius $e_{\max }\left(e_{i}{ }^{-1}-1 / 2\right)$ around $p_{i}$ and a circle of radius $e_{\max }\left(e_{j}{ }^{-1}-1 / 2\right)$ around $p_{j}$. We need to check that the condition $|a-b| \leq c$ holds. Assuming that $e_{i} \leq e_{j}$, we have

$$
\begin{aligned}
c-|a-b| & \geq \frac{e_{k}}{e_{i} e_{j}}-e_{\max }\left|e_{i}{ }^{-1}-e_{j}{ }^{-1}\right| \\
& =\frac{1}{e_{i} e_{j}}\left(e_{k}-e_{\max }\left(e_{j}-e_{i}\right)\right) \\
& \geq \frac{1}{e_{i} e_{j}}(1-1.5152(1.5152-1)) \\
& \geq 0
\end{aligned}
$$

where the second-to-last line used the fact that $1 \leq e_{i} \leq e_{j} \leq 1.5152$. Hence we do in fact have $|a-b| \leq c$; i.e., it is not the case that one circle lies entirely inside the other. 
Note that it $i s$ entirely possible that $e_{k} /\left(e_{i} e_{j}\right)$ will be greater than $e_{\max }\left(e_{i}{ }^{-1}+\right.$ $\left.e_{j}{ }^{-1}-1\right)$, i.e. that the two circles don't overlap at all. In this case, $l_{i, j, k}$ will simply equal 0 since overlapApprox $(a, b, a+b)=0$ for all non-negative $a$ and $b$.

Now suppose that the manifold $N$ possesses an $(i, j, k)$-triple where $i, j$, and $k$ are all elements of $\{1,2,3\}$. Then there will exist orthocenters $p_{i}$ and $p_{j}$ corresponding to horoballs $B_{i} \in \mathcal{O}(i)$ and $B_{j} \in \mathcal{O}(j)$ such that $d\left(B_{i}, B_{j}\right)=o(k)$, and similarly for each cyclic permutation of $i, j$, and $k$. In other words, an $(i, j, k)$-triple with $i$, $j, k \in\{1,2,3\}$ can cause up to three different overlaps in $\partial B_{\infty} / H$. But there will be no overlaps between the circles that we have constructed that do not come from such a triple. In other words we have established the following:

Lemma 5.4. Suppose that $N$ possesses horoball triples of the form $\left(i_{1}, j_{1}, k_{1}\right), \ldots$, $\left(i_{s}, j_{s}, k_{s}\right)$, where $i_{r}, j_{r}, k_{r} \in\{1,2,3\}$ for each $r=1, \ldots, s$. Furthermore, suppose every such triple in $N$ appears in the above list exactly once. Then

$$
\operatorname{Area}\left(\partial B_{\infty} / H\right) \geq A_{0}-\sum_{r=1}^{s} l_{i_{r}, j_{r}, k_{r}}+l_{j_{r}, k_{r}, i_{r}}+l_{k_{r}, i_{r}, j_{r}} .
$$

Once we have a bound on the area of $\partial B_{\infty} / H$, we can compute the volume of $B_{\infty} / H$ and thereby construct a bound on the volume of $N$ exactly as in the previous section. Recall that in the previous section we "inflated" the cusp neighborhood to get a larger neighborhood $C / H$ which was only immersed in $N$, and then we subtracted the volume of the regions where $C / H$ extended beyond the Ford domain corresponding to $B_{\infty} / H$. This allowed us to improve our volume bound by accounting for some of the volume of $N-B_{\infty} / H$. We will do the same here, "inflating" our cusp neighborhood by replacing $B_{\infty}$ with a new horoball $C$ centered at infinity but with Euclidean height $1 / e_{\max }$. Then we have the following:

Lemma 5.5. Under the same assumptions as in Lemma 5.4.

$$
\begin{aligned}
\operatorname{Vol}(N) \geq & \frac{e_{\max }^{2}}{2}\left(A_{0}-\sum_{r=1}^{s} l_{i_{r}, j_{r}, k_{r}}+l_{j_{r}, k_{r}, i_{r}}+l_{k_{r}, i_{r}, j_{r}}\right) \\
& -\pi\left(-3+e_{\max }^{2}\left(1+e_{2}{ }^{-2}+e_{3}{ }^{-2}\right)+\log \left(\frac{e_{2}^{2} e_{3}^{2}}{e_{\max }{ }^{2}}\right)\right) .
\end{aligned}
$$

Unfortunately one very quickly discerns that the volume bounds obtained by the above lemma simply aren't large enough for our purposes, particularly when $e_{2}, e_{3}$, and $e_{4}$ are small. For example, if $e_{2}=e_{3}=e_{4}=1$, then one can quickly determine that $A_{0}=3 \pi / 2$ and that all the $l_{i, j, k}$ 's are 0 , resulting in a volume bound of $3 \pi / 4$, which is less than 2.848. Thus we need to find more area in the case where $e_{4}$ is small. Since a small value of $e_{4}$ implies that the $\mathcal{O}(4)$-horoballs will be close to $B_{\infty}$, it is natural to try to construct circles around the orthocenters of the $\mathcal{O}(4)$-horoballs to increase our estimate of the area of $B_{\infty} / H$.

So suppose $e_{4}<1.5152$ (so $\left.e_{\max }=e_{4}\right)$. We construct a circle of radius $1 /\left(e_{4} e_{2}\right)-$ $e_{4} / e_{2}+e_{4} / 2$ around the orthocenter of each of the two horoballs in $\mathcal{O}(4)$. The reasoning behind the choice of this particular radius is as follows. If $q_{4}$ is the orthocenter of such a horoball, and if $p_{i}$ is the orthocenter of $B_{i} \in \mathcal{O}(i)$ for $i \in$ $\{2,3,4\}$ (assume $p_{i} \neq q_{4}$ ), we want the circles constructed around $q_{4}$ and $p_{i}$ to have 
disjoint interiors. Since $d_{E}\left(p_{i}, q_{4}\right) \geq 1 /\left(e_{i} e_{4}\right)$ by Lemma 3.4 this means that when $i=2$ or 3 we require

$$
\frac{1}{e_{i} e_{4}}-\left(\frac{1}{e_{4} e_{2}}-\frac{e_{4}}{e_{2}}+\frac{e_{4}}{2}\right)-\left(\frac{e_{4}}{e_{i}}-\frac{e_{4}}{2}\right) \geq 0,
$$

and when $i=4$ we require

$$
\frac{1}{e_{4}{ }^{2}}-2\left(\frac{1}{e_{4} e_{2}}-\frac{e_{4}}{e_{2}}+\frac{e_{4}}{2}\right) \geq 0 .
$$

The first inequality is equivalent to $\left(e_{2}{ }^{-1}-e_{i}{ }^{-1}\right)\left(e_{4}-e_{4}{ }^{-1}\right) \geq 0$, which is clearly true for $i=2$ or 3 . The second inequality simplifies to

$$
\frac{e_{4}-1}{e_{4}{ }^{2} e_{2}}\left(2 e_{4}^{2}+2 e_{4}-e_{2}\left(e_{4}^{2}+e_{4}+1\right)\right) \geq 0 .
$$

But $1 \leq e_{2} \leq e_{4}$, and the polynomial $2 x^{2}+2 x-x\left(x^{2}+x+1\right)$ is always positive for $1 \leq x \leq 1.5152$, and hence each factor on the left-hand side of the above inequality is always non-negative, proving the inequality.

Hence our new circles will not intersect each other, nor will they intersect the previous circles that were created around the orthocenters of the $\mathcal{O}(2)$ or $\mathcal{O}(3)$ horoballs. Now suppose $q_{4}$ is the orthocenter of $B_{4} \in \mathcal{O}(4)$ as before, and suppose $p_{1}$ is the orthocenter of a horoball $B_{1} \in \mathcal{O}(1)$. Suppose further that $d\left(B_{1}, B_{4}\right) \geq o(2)$, and that hence $d_{E}\left(p_{1}, q_{4}\right) \geq e_{2} / e_{4}$ by Lemma 3.4. We wish to know if the circles we've constructed around $p_{1}$ and $q_{4}$ will overlap; that is, we wish to verify the inequality

$$
\begin{aligned}
\frac{e_{2}}{e_{4}}-\left(\frac{1}{e_{4} e_{2}}-\frac{e_{4}}{e_{2}}+\frac{e_{4}}{2}\right)-\frac{e_{4}}{2} & \geq 0 \\
\Leftrightarrow \frac{e_{2}-1}{e_{4} e_{2}}\left(e_{2}+1-e_{4}{ }^{2}\right) & \geq 0 .
\end{aligned}
$$

This inequality is not always true in the domain $1 \leq e_{2} \leq e_{4} \leq 1.5152$, particularly when $e_{2}$ is small and $e_{4}$ is large. However, the inequality does hold when we need it to hold, namely when $e_{2}$ and $e_{4}$ are both small. Hence in what follows we will make the additional assumption that $e_{2}+1 \geq e_{4}{ }^{2}$, and we take it for granted that if this assumption fails we don't need the extra area anyway.

With the additional assumption, we now have circles constructed around the orthocenters of the $\mathcal{O}(4)$-horoballs which don't intersect each other, which don't intersect the circles around the centers of the $\mathcal{O}(2)$-horoballs and $\mathcal{O}(3)$ horoballs, and which may intersect the circles constructed around an $\mathcal{O}(1)$ horoball but only if that horoball is less than $o(2)$ away from the $\mathcal{O}(4)$-horoballs. In other words, the new circles do not intersect themselves or any of the previous circles unless $N$ contains one or more $(1,1,4)$-triples. If $N$ contains exactly one such triple, there will be two overlaps to account for, while if $N$ contains two or more such triples, we have a geometric Mom-2 structure. Thus we can conclude the following:

Lemma 5.6. If $e_{4} \leq 1.5152$ and $e_{2}+1 \geq e_{4}{ }^{2}$, then either $N$ contains a geometric Mom-2 structure consisting of two $(1,1,4)$-triples or else the area estimate of Lemma 5.4 can be increased by

$$
2 \pi\left(\frac{1}{e_{4} e_{2}}-\frac{e_{4}}{e_{2}}+\frac{e_{4}}{2}\right)^{2}-2 \text { overlapApprox }(a, b, c),
$$

where $a=e_{4} / 2, b=1 /\left(e_{4} e_{2}\right)-e_{4} / e_{2}+e_{4} / 2$, and $c=1 / e_{4}$. 
Note that $a$ and $b$ are the radii of the circles constructed around the orthocenters of the $\mathcal{O}(1)$-horoballs and $\mathcal{O}(4)$-horoballs, respectively, while $c$ is the minimum possible distance between those orthocenters in the presence of a $(1,1,4)$-triple. Also we are implicitly assuming that the conditions of Lemma 3.12 are met, namely that $|a-b| \leq c \leq a+b$. Fortunately the left-hand inequality simplifies to $\left(e_{2}+\right.$ $\left.1-e_{4}{ }^{2}\right) /\left(e_{2} e_{4}\right) \geq 0$, which is true by assumption, while the right-hand inequality simplifies to $\left(e_{4}^{2}-1\right)\left(e_{2}-1\right) /\left(e_{2} e_{4}\right) \geq 0$.

We can also update our volume bounds:

Lemma 5.7. If $e_{4} \leq 1.5152$ and $e_{2}+1 \geq e_{4}{ }^{2}$, then either $N$ contains a geometric Mom-2 structure consisting of two $(1,1,4)$-triples or else the volume estimate of Lemma 5.5 can be increased by

$$
\frac{e_{4}^{2}}{2}\left(2 \pi\left(\frac{1}{e_{4} e_{2}}-\frac{e_{4}}{e_{2}}+\frac{e_{4}}{2}\right)^{2}-2 \text { overlapApprox }(a, b, c)\right),
$$

where $a=e_{4} / e_{2}, b=1 /\left(e_{4} e_{2}\right)-e_{4} / e_{2}+e_{4} / 2$, and $c=1 / e_{4}$.

We now have in principle a procedure for proving Proposition 5.1. First, enumerate every possible combination of triples which does not include a geometric Mom-2 or Mom-3 structure which is torus-friendly. Second, for each combination construct the volume bounds from Lemma 5.5 and Lemma 5.7 above, which will be a function of $e_{2}, e_{3}$, and $e_{4}$. Third, show that each such volume bound never attains a value below 2.848 .

The first step is the simplest, and in fact we can make it even simpler: we do not need to enumerate every possible combination of triples that do not include a Mom- $n$, merely the maximal ones, since adding additional triples only decreases the area bounds in Lemma 5.4 and Lemma 5.6. There are a total of eighteen maximal combinations of triples that must be considered:

- $(1,1,2),(1,1,3)$

- $(1,1,2),(1,3,3)$

- $(1,1,2),(2,2,3)$

- $(1,1,2),(2,3,3)$

- $(1,2,2),(1,1,3)$

- $(1,2,2),(1,3,3)$

- $(1,2,2),(2,2,3)$

- $(1,2,2),(2,3,3)$

- $(1,1,3),(2,2,3)$

- $(1,1,3),(2,3,3)$

- $(1,3,3),(2,2,3)$

- $(1,3,3),(2,3,3)$

- $(1,1,2),(1,2,3),(1,2,3)$

- $(1,2,2),(1,2,3),(1,2,3)$

- $(1,1,3),(1,2,3),(1,2,3)$

- $(1,3,3),(1,2,3),(1,2,3)$

- $(2,2,3),(1,2,3),(1,2,3)$

- $(2,3,3),(1,2,3),(1,2,3)$

The second step of the procedure is also simple; it can in fact be automated by a few lines of Mathematica code ([M2]). 
The third step, however, is daunting due to the complicated nature of the volume bounds that result from Lemmas 5.5 and 5.7. The expressions resulting from these two lemmas defy analysis by hand.

To handle these complicated expressions, we resort to computer assistance. Specifically, we use rigorous floating-point arithmetic of the type used in [GMT. This is not standard interval arithmetic, so we take a moment to review the techniques involved here.

Definition 5.8. An affine 1-jet $F=\left(f_{0} ; f_{1}, f_{2}, f_{3} ; f_{\epsilon}\right)$ consists of the set of all functions $f:[-1,1]^{3} \rightarrow \mathbb{R}$ such that $\left|f\left(x_{1}, x_{2}, x_{3}\right)-\left(f_{0}+\sum f_{i} x_{i}\right)\right| \leq f_{\epsilon}$ for all $\left(x_{1}, x_{2}, x_{3}\right) \in[-1,1]^{3}$. (Note that we require $f_{\epsilon} \geq 0$.)

Note that in GMT, complex 1-jets were used; however we only require real numbers here. GMT showed how, given two affine 1-jets $F$ and $G$, a computer which meets IEEE standards for floating-point arithmetic can compute an affine 1-jet $H=\left(h_{0} ; h_{1}, h_{2}, h_{3} ; h_{\epsilon}\right)$ which "equals $F+G$ ", in the sense that $f+g \in H$ for each $f \in F$ and $g \in G$. Specifically, let $h_{i}=f_{i}+g_{i}$ for $i=0,1,2$, and 3, and let

$$
h_{\epsilon}=\left(1+\epsilon_{a}\right)\left(\epsilon_{t}+\epsilon_{f}\right) \text {, }
$$

where

$$
\begin{aligned}
\epsilon_{t} & =f_{\epsilon}+g_{\epsilon}, \\
\epsilon_{f} & =\frac{E P S}{2}\left(\left(\left|f_{0}+g_{0}\right|+\left|f_{1}+g_{1}\right|\right)+\left(\left|f_{2}+g_{2}\right|+\left|f_{3}+g_{3}\right|\right)\right), \\
\epsilon_{a} & =3 E P S .
\end{aligned}
$$

Here $\epsilon_{t}$ accounts for the "Taylor error", i.e. the maximum possible sup-norm distance between $f+g$ and the linear function represented by $h$ as $f$ and $g$ vary over $F$ and $G$ respectively. Since we're only computing a linear function of $f$ and $g$ anyway, $\epsilon_{t}$ only needs to account for the error carried over from the operands $F$ and $G$; for more complicated operations this term will be more significant. The quantity $\epsilon_{f}$ accounts for the floating-point error that may accrue from the calculation of $h_{0}$ through $h_{3}$. Here EPS is a (small) computer-dependant constant that measures the granularity of the set of real numbers that the computer is capable of representing. Roughly, $1+E P S$ will be the smallest real number strictly greater than 1 which has a floating-point representation on the computer in question. Finally $\epsilon_{a}$ accounts for the floating-point error that may accrue from calculating $\epsilon_{t}+\epsilon_{f}$. For any operation involving affine 1-jets, $\epsilon_{a}$ will always be of the form $n E P S$ where $n$ is an integer roughly proportional to the base-2 logarithm of the number of arithmetic operations necessary to compute $\epsilon_{t}+\epsilon_{f}$. Constructed in this way, the error term $h_{\epsilon}$ will be large enough to account for the original error terms $f_{\epsilon}$ and $g_{\epsilon}$ and for the floating-point error that might accrue from calculating the terms of $H$. Similar constructions exist for " $-F$ ", " $F G$ ", and " $F / G$ " provided the range of $g$ does not contain 0 for any $g \in G$.

For more specific details on this process, we refer the reader to Sections 5 and 6 of [GMT, which describes the theory behind these formulas and provides numerous examples.

Now suppose that we have a rational polynomial $p\left(x_{1}, x_{2}, x_{3}\right)$ and we wish to compute the range of possible values of $p$ over a box $I_{1} \times I_{2} \times I_{3}$ in $\mathbb{R}^{3}$. It is not possible to compute the exact range of possible values by computer due to floatingpoint error. However affine 1-jets do make it possible to rigorously determine an 
interval which must contain the range of possible values, as follows. Note that for clarity's sake we start with a simplified version of the procedure and fill in certain troublesome details later.

Define $X_{1}$ to be the affine 1 -jet $\left(a_{1} ; b_{1}, 0,0 ; 0\right)$, where $x \mapsto a_{1}+b_{1} x$ is the unique non-decreasing linear bijection from $[-1,1]$ to the interval $I_{1}$. Define $X_{2}=$ $\left(a_{2} ; 0, b_{2}, 0 ; 0\right)$ and $X_{3}=\left(a_{3} ; 0,0, b_{3} ; 0\right)$ similarly. Then compute the affine 1 -jet $p\left(X_{1}, X_{2}, X_{3}\right)$ using the constructions in GMT in place of the usual arithmetic operations, and let $P=\left(p_{0} ; p_{1}, p_{2}, p_{3} ; p_{\epsilon}\right)$ denote the result. If $\left(x_{1}, x_{2}, x_{3}\right) \in I_{1} \times I_{2} \times I_{3}$, then trivially

$$
\left(x_{1}, x_{2}, x_{3}\right)=\left(f_{1}(u, v, w), f_{2}(u, v, w), f_{3}(u, v, w)\right),
$$

where $(u, v, w) \in[-1,1]^{3}$ and $f_{i} \in X_{i}$ for $i=1,2$, and 3. (Specifically, we may choose $f_{i}(x)=a_{i}+b_{i} x$.) Therefore $p\left(x_{1}, x_{2}, x_{3}\right)$ must lie in the range of $p\left(f_{1}, f_{2}, f_{3}\right)$, which is an element of $P$. Therefore

$$
p\left(x_{1}, x_{2}, x_{3}\right) \in\left[p_{0}-\left|p_{1}\right|-\left|p_{2}\right|-\left|p_{3}\right|-p_{\epsilon}, p_{0}+\left|p_{1}\right|+\left|p_{2}\right|+\left|p_{3}\right|+p_{\epsilon}\right] .
$$

In practice, there are several complications. First, the above construction implicitly assumes that the coefficients $a_{i}$ and $b_{i}$ have exact binary representations. If they do not, then the affine 1-jets $X_{i}$ must be modified to have a non-zero $\epsilon$ term, representing the sup-norm distance between $a_{i}+b_{i} x$ and the actual unique non-decreasing linear bijection from $[-1,1]$ to $I_{i}$. In practice it is usually simpler to replace $I_{i}$ with a slightly larger interval whose endpoints do in fact have exact binary representations. Second, computing whether or not $p\left(x_{1}, x_{2}, x_{3}\right)$ lies in the interval described is itself a floating-point operation and may introduce error. This can be dealt with using similar techniques to those used in calculating " $F+G$ " in the first place. One final complication is that this whole technique will only be effective if $p$ is very simple or if the intervals $I_{i}$ are very small. If $p$ is complicated (and the functions we're interested in are very complicated), then it is usually necessary to subdivide $I_{1} \times I_{2} \times I_{3}$ into much smaller sub-boxes to achieve any kind of accuracy.

Nevertheless, we wish to apply the above techniques to compute a range of possible values for the volume bounds produced by Lemmas 5.5 and 5.7 if the computed ranges never include values less than or equal to 2.848 , then we'll be done. Unfortunately those volume bounds are not expressed as rational polynomials; we also need to be able to rigorously compute both natural logarithms and the minimum function, two operations that were never implemented in GMT.

Fortunately while the natural logarithm is not a rational polynomial, all of its derivatives are. This makes it possible to rigorously compute logarithms by using a Taylor approximation to $\log x$ at $x=1$, and using Taylor's theorem to compute an exact upper bound on the difference between $\log x$ and the polynomial approximation. Since Taylor's theorem expresses this difference in terms of the derivatives of $\log x$ it is possible to incorporate it into the "Taylor error term" $\epsilon_{t}$ of the resulting affine 1-jet and still determine correct upper bounds for the corresponding $\epsilon_{f}$ and $\epsilon_{a}$ terms. Repeated use of the relation $\log (a x)=\log a+\log x$ (where $a \neq 1$ is just an arbitrary positive constant; $9 / 8$ was used in our implementation) allows the program to restrict its use of the Taylor approximation to regions close to $x=1$, where the approximation is the most accurate.

It is tempting at this point to try and implement other non-polynomial functions in this way. For example, consider the function overlapArea $(a, b, c)$ from Definition 
3.11 it is composed of polynomials, square roots, and the function $\operatorname{acos}(x)$. Square roots were successfully implemented using affine 1-jets in [GMT], and the derivatives of $\operatorname{acos}(x)$ are square roots of rational polynomials, so in theory it is possible to implement the function overlapArea with affine 1-jets. In practice, unfortunately, this works poorly. The function $f(x)=\operatorname{acos}(x)-x \sqrt{1-x^{2}}$ which is used in the calculation of overlapArea is composed of two functions which have vertical tangent lines at the points $x= \pm 1$. When computing a function using affine 1 -jets, the size of the resulting error term will always be proportional to the derivative of the function being computed; as a consequence, computing $f(x)$ for affine 1-jets which include $x= \pm 1$ in their range in practice causes the error term to grow so large as to make the entire 1-jet useless. This is the entire reason for the existence of Lemma 3.12. While overlapApprox $(a, b, c)$ is only an approximation to overlapArea $(a, b, c)$, as a polynomial function it is far more useful in this kind of computation.

Continuing, there remains one function to be implemented: a function $\operatorname{Max} 0(F)$ such that if $f \in F$ and $H=\operatorname{Max} 0(F)$, then $\max (f, 0) \in H$. (Then the identity $\min (f, g)=f-\max (f-g, 0)$ can be used to compute minimums. $)$ If the computer can rigorously determine that 0 does not lie in the range of $f$ for any $f \in F$, then $\operatorname{Max} 0(F)$ equals either $F$ itself if $f_{0}>0$ or else $\operatorname{Max} 0(F)=(0 ; 0,0,0 ; 0)$ if $f_{0}<0$. If the computer cannot exclude the possibility that 0 lies in the range of some $f \in F$, then $\operatorname{Max} 0(F)$ is defined to be equal to the 1 -jet $(s ; 0,0,0 ; s)$, where

$$
s=\frac{1}{2}(1+3 \operatorname{EPS})\left(f_{0}+\left(\left(\left|f_{1}\right|+\left|f_{2}\right|\right)+\left(\left|f_{3}\right|+f_{\epsilon}\right)\right) .\right.
$$

(The factor of $(1+3 E P S)$ is there to account for floating-point error that may accrue during the calculation of the rest the expression.) Note that while this definition is technically correct in that $\max (f, 0)$ will lie in this affine 1 -jet for all $f \in F$, from a practical standpoint it is a terrible definition as $s$ is almost guaranteed to be much, much larger than the original error term $f_{\epsilon}$. Fortunately (and unlike the case with $\operatorname{acos}(x)$ and square roots, above) in practice the cases where the $\operatorname{Max} 0$ function had to be called on affine 1-jets that may have contained 0 in their ranges were rare enough not to cause significant problems.

With these tools in hand we now consider the following:

Theorem 5.9. Suppose $N$ is a one-cusped hyperbolic 3-manifold with $\operatorname{Vol}(N) \leq$ 2.848. Then $e_{2} \leq 1.4751$, and furthermore $N$ contains a geometric Mom-n structure which either

- is a geometric Mom-2 structure incorporating only the orthoclasses $\mathcal{O}(1)$ and $\mathcal{O}(2)$ and triples constructed from those three orthoclasses, or

- incorporates only the orthoclasses $\mathcal{O}(1), \mathcal{O}(2)$, and $\mathcal{O}(3)$, and triples constructed from those three orthoclasses, and is torus-friendly, and in addition we have $e_{3} \leq 1.5152$, or

- incorporates the orthoclasses $\mathcal{O}(1)$ and $\mathcal{O}(4)$ and a pair of $(1,1,4)$-triples, and in addition we have $e_{4} \leq 1.5152$.

The proof of Theorem [5.9] using rigorous computer assistance, proceeds as follows. If $e_{2}>1.4751$, then $\operatorname{Vol}(N)>2.848$ by the results of the previous chapter. So, suppose otherwise. Furthermore, suppose that $N$ does not contain a geometric Mom- $n$ of one of the three types described. In particular, by Proposition 4.1 we may assume that $e_{3} \leq 1.5152$, and we may assume that $N$ contains at most one $(1,1,4)$-triple unless $e_{4}>1.5152$. 
Then let $\left(i_{1}, j_{1}, k_{1}\right), \ldots,\left(i_{s}, j_{s}, k_{s}\right)$ be the complete list of triples in $N$ satisfying the condition that $i_{r}, j_{r}, k_{r} \in\{1,2,3\}$ for all $r$. This list of triples must be a subcollection of one of the eighteen collections listed above. For each of those eighteen collections of triples, we can construct a lower bound on the volume of $N$ via Lemma 5.5, which we will call $f_{1}\left(e_{2}, e_{3}, e_{4}\right)$. In addition, Lemma 5.7 provides a second, improved lower bound in the case where $e_{4} \leq 1.5152$ and $e_{2}+1 \geq e_{4}{ }^{2}$; call this function $f_{2}\left(e_{2}, e_{3}, e_{4}\right)$. These bounds depend solely on the parameters $e_{2}, e_{3}$, and $e_{4}$ and can be computed using only the four basic arithmetic operations, logarithms, the minimum function, and some constants. Furthermore we may assume that $\left(e_{2}, e_{3}, e_{4}\right)$ lies in a compact subset of $\mathbb{R}^{3}$. Technically we have not established an upper bound on $e_{4}$, but if $e_{4}>1.5154$, then we may simply replace $e_{4}$ with 1.5152 in $f_{1}$ and still obtain a valid lower bound on the volume of $N$. We cannot do the same with $f_{2}$, but in practice $f_{2}$ is only needed to improve the volume bound for small values of $e_{4}$. Subdividing this compact region into a sufficiently small number of pieces and using the rigorous floating-point arithmetic techniques described above, we can rigorously demonstrate that $\max \left(f_{1}, f_{2}\right)$ never attains a value less than or equal to 2.848 .

This approach has been successfully implemented (플 $)$ and used to prove Theorem [5.9 and hence Proposition 5.1] The resulting program requires approximately 80 minutes to establish that $\max \left(f_{1}, f_{2}\right)>2.848$ on the parameter space $1 \leq e_{2} \leq 1.4751, e_{2} \leq e_{3} \leq e_{4} \leq 1.5152$ in each of the 18 cases. To do so the program subdivides each dimension of the parameter space into $2^{8}$ subintervals. The program also establishes that $f_{1}>2.848$ whenever $1 \leq e_{2} \leq 1.4751$, $e_{2} \leq e_{3} \leq 1.5152$, and $e_{4}=1.5152$, requiring approximately 10 seconds to do so; the number of dimensions in the parameter space has a tremendous effect on the running time. The programs themselves consist of just under 4800 lines of $\mathrm{C}++$ code (close to half of which was generated automatically by a short Mathematica program), running in Redhat Linux on a four-processor PC.

Remark 5.10. The methods of this section can be used to analyze areas of maximal cusp tori. In particular, the authors have used a modified version of the code described in this section to show that if $N$ is a one-cusped hyperbolic 3-manifold which does not possess a torus-friendly geometric Mom- $n$ structure for $n=2$ or 3 , then the area of the maximal cusp torus is greater than or equal to 3.78. Presumably, the techniques used in [M] could be used to study the case of manifolds which do possess torus-friendly geometric Mom- $n$ structures and complete the analysis. Similar techniques have also been used in [LM] to study the maximal number of exceptional Dehn surgeries in a one-cusped hyperbolic 3-manifold.

\section{Embedding A GeOMEtric Mom- $n$}

Theorem 5.9 establishes conditions under which we can assume that a one-cusped hyperbolic 3-manifold $N$ possesses a geometric Mom-2 or Mom-3 structure. Now what we wish to do is thicken the cellular complex $\Delta$ associated to that structure to obtain a full topological internal Mom- $n$ structure as defined in GMM2. Examining that definition, we see that there are three obstacles to this. The first is that there is no guarantee that $\Delta$ is embedded in $N$; this is the obstacle we will address in this section. The second obstacle is that the complement of $\Delta$ in $N$ may have components whose boundaries are not tori, which violates the definition of an internal Mom- $n$ structure; this obstacle will be addressed in the next section. The 
final obstacle, namely determining whether or not $\Delta$ is "full", will be tackled in Section 8 . We proceed with the question of embeddedness now; specifically we will prove the following:

Theorem 6.1. In Theorem 5.9, we may assume that the geometric Mom-n structure obtained has the property that the cellular complex $\Delta=T \cup\left\{\lambda_{i}\right\} \cup\left\{\sigma_{j}\right\}$ is embedded, where $\left\{\lambda_{i}\right\}$ is the set of 1-cells which are the projections in $N$ of the shortest arcs joining the horoballs which form the orthopair classes of the Mom-n, and where $\left\{\sigma_{j}\right\}$ is the set of 2-cells which are the projections in $N$ of the totally geodesic surfaces spanning the triples of horoballs in the geometric Mom-n structure and the arcs between them.

For the sake of notation, define the $\mathcal{O}(n)$-edge to be the image in $N$ of the shortest arc between any two horoballs which constitute an element of $\mathcal{O}(n)$. All of the lemmas in this chapter will assume that $N$ is a one-cusped hyperbolic 3manifold.

Lemma 6.2. If the $\mathcal{O}(n)$-edge intersects the cusp torus for any $n$, then $e_{n}>1.5152$.

Proof. Any edge which intersects the cusp torus lifts to an arc contained in a line which intersects the boundary of $B_{\infty}$, neither of whose endpoints are at infinity. Such a line clearly must be a half-circle of diameter $\geq 2$. But such a line must also join the centers of two horoballs and hence by Lemma 3.4 we must have $e_{k} /\left(e_{m} e_{n}\right) \geq$ 2 for some $k \in\{1,2,3\}$ and for some $m$ and $n$. This implies that $e_{k} \geq 2>$ 1.5152 .

Lemma 6.3. If any two-cell in $\Delta$ corresponding to an $(n, m, k)$-triple intersects the cusp torus, then one of $e_{n}, e_{m}$, or $e_{k}$ is greater than 1.5152 .

Proof. A two-cell which intersects the cusp torus lifts to a totally geodesic two-cell contained in an ideal hyperbolic triangle which intersects $B_{\infty}$, whose ideal vertices are the centers of three horoballs $A, B, C$, with the property that $d(A, B)=o(n)$, $d(B, C)=o(m)$, and $d(C, A)=o(k)$. There are two possibilities. If one of the three edges of this ideal triangle also intersects $B_{\infty}$, then the previous lemma applies and we're done. So suppose none of the three edges intersects $B_{\infty}$, but instead the triangle intersects $B_{\infty}$ at some point in its interior.

Let $p$ be the highest point of the ideal triangle as viewed in the upper half-space model (here "highest" refers to the Euclidean or visual height), and let $q$ be the highest point of the entire hyperbolic plane containing this triangle. Clearly if $p \neq q$, then $p$ must be some point on an edge of the ideal triangle as close to $q$ as possible, contradicting our supposition. So $p=q$ and $q$ is contained in the ideal triangle. Now view the entire picture from the point at infinity: from above, the ideal triangle projects onto a Euclidean triangle in $B_{\infty}$ joining the orthocenters of $A$, $B$, and $C$, and $q$ projects to the circumcenter of this triangle. Since the projection of $q$ lies in the interior of the triangle, and since the circumradius of the triangle is $\geq 1$, one of the sides of the triangle must have length greater than or equal to $\sqrt{2-2 \cos \frac{2 \pi}{3}}=\sqrt{3}$. Without loss of generality, assume that the side "from $B$ to $C$ " has this property. Therefore by Lemma 3.4 as before we have $e_{k} /\left(e_{b} e_{c}\right) \geq \sqrt{3}$ for some $k \in\{1,2,3\}$ and some $b$ and $c$, and hence $e_{k} \geq \sqrt{3}>1.5152$.

The next step is to establish whether or not any of the $\mathcal{O}(n)$-edges contained in $\Delta$ can intersect one another. Some of the following lemmas are stronger than 
necessary to prove that this is not the case, but the stronger results will be used later in the argument. We begin with some definitions to simplify notation later:

Definition 6.4. Let $\delta_{1}=0.15, \delta_{2}=\cosh ^{-1}\left(1.5152^{-1}+1.5152^{-2}\right)=0.4337 \ldots$, and $\delta_{3}=\cosh ^{-1}(2 / 1.5152)=0.7800 \ldots$

Note that the value of $\delta_{1}$ comes from Lemma 3.7. The values of $\delta_{2}$ and $\delta_{3}$ are motivated by the following application of Corollary 3.6 .

Lemma 6.5. Suppose $A, B, C, D$, and the various distances between them are defined as in Corollary 3.6. In particular suppose that $d(A, C)=o(m), d(B, D)=$ $o(n)$, and suppose that $x$ is the shortest distance between the line joining the centers of $A$ and $C$ and the line joining the centers of $B$ and $D$. Suppose further that $\max \left(e_{m}, e_{n}\right) \leq 1.5152$. If $x<\delta_{2}$, then all four of the distances $o(h)=$ $d(A, B), o(j)=d(B, C), o(k)=d(C, D)$, and $o(l)=d(D, A)$ are strictly less than $\min (o(m), o(n))$. If $x<\delta_{3}$, then at least three of those four distances are strictly less than $\min (o(m), o(n))$.

Proof. Without loss of generality suppose that $o(m) \leq o(n)$, and that therefore $e_{m} \leq e_{n} \leq 1.5152$.

Suppose that $x<\delta_{2}$ and that $e_{h} \geq e_{m}$. Then we have the following:

$$
\begin{aligned}
e_{m}+1 & \leq e_{h} e_{k}+e_{j} e_{l} \\
& =e_{m} e_{n} \cosh x \\
& <\left(1.5152^{-1}+1.5152^{-2}\right) e_{m} e_{n}
\end{aligned}
$$

where the middle step used the result of Corollary 3.6. Dividing both sides by $e_{m}$ and using the fact that $e_{m} \leq e_{n} \leq 1.5152$ we get

$$
1+\frac{1}{1.5152} \leq 1+\frac{1}{e_{m}}<\left(1.5152^{-1}+1.5152^{-2}\right) e_{n} \leq 1+\frac{1}{1.5152},
$$

which is a contradiction. Hence $e_{h}<e_{m}$, and similarly for $e_{j}, e_{k}$, and $e_{l}$.

Now suppose that $x<\delta_{3}$. If $e_{h} \geq e_{m}$ and $e_{k} \geq e_{m}$, then we have

$$
\begin{aligned}
e_{m}^{2}+1 & \leq e_{h} e_{k}+e_{j} e_{l} \\
& =e_{m} e_{n} \cosh x \\
& <\frac{2}{1.5152} e_{m} e_{n}
\end{aligned}
$$

Again, divide both sides by $e_{m}$. Then using $e_{n} \leq 1.5152$ and the arithmetic meangeometric mean inequality we get

$$
2 \leq e_{m}+\frac{1}{e_{m}}<\frac{2}{1.5152} e_{n} \leq 2
$$

which is a contradiction. On the other hand, if $e_{h} \geq e_{m}$ and $e_{j} \geq e_{m}$, then we have

$$
\begin{aligned}
e_{m}+e_{m} & \leq e_{h} e_{k}+e_{j} e_{l} \\
& =e_{m} e_{n} \cosh x \\
& <\frac{2}{1.5152} e_{m} e_{n} .
\end{aligned}
$$

Dividing both sides by $e_{m}$ gives $2<\frac{2}{1.5152} e_{n} \leq 2$, a contradiction. Hence by symmetry no two of $e_{h}, e_{j}, e_{k}$, and $e_{l}$ can be greater than or equal to $e_{m}$; therefore at least three of them are strictly less than $e_{m}$. This proves the lemma. 
Lemma 6.6. The $\mathcal{O}(1)$-edge does not intersect or pass within less than $\delta_{3}$ of any $\mathcal{O}(n)$-edge where $e_{n} \leq 1.5152$.

Proof. Apply Lemma 6.5 with $m=1$ and $x<\delta_{3}$ to conclude that either three of $\{o(h), o(j), o(k), o(l)\}$ are less than $o(1)=0$, which is ridiculous, or else $e_{n}>1.5152$, a contradiction.

Lemma 6.7. If the $\mathcal{O}(2)$-edge intersects the $\mathcal{O}(3)$-edge, or passes within less than $\delta_{2}$ of it, and if $e_{3} \leq 1.5152$, then there exists a geometric Mom-2 structure consisting only of $(1,1,2)$-triples.

Proof. Applying Lemma 6.5 we get that $e_{h}=e_{j}=e_{k}=e_{l}=e_{1}$. Hence $d(A, C)=$ $d(A, D)=d(B, C)=d(B, D)=0$ and therefore $\{A, B, C\}$ and $\{A, C, D\}$ are both $(1,1,2)$-triples. They cannot be equivalent under the action of $\pi_{1}(N)$, because any element of the group which maps one triple to the other would have to map the pair $\{A, C\}$ to itself, and hence be either elliptic or the identity, a contradiction. Therefore these two triples are distinct and constitute a geometric Mom-2 structure, completing the proof.

Lemma 6.8. If $e_{n} \leq 1.5152$, then the $\mathcal{O}(n)$-edge neither intersects itself nor passes within $\delta_{1}$ of itself.

Proof. This is a direct corollary of Lemma 3.7

The three preceding lemmas show that if $N$ contains a geometric Mom-2 or Mom3 structure of one of the types described in Theorem [5.9, then the $\mathcal{O}(n)$-edges of the complex $\Delta$ are embedded in $N$ and do not intersect one another, unless the Mom- $n$ structure includes the $\mathcal{O}(3)$-edge. In that case, either the $\mathcal{O}(n)$-edges of the complex $\Delta$ are embedded and do not intersect or else there exists another geometric Mom- $n$ structure, specifically a Mom-2 structure consisting only of $(1,1,2)$-triples. In other words, either the edges in question are embedded and do not intersect, or else we can find a simpler Mom- $n$ structure; hence we may argue by induction that $N$ must possess a geometric Mom- $n$ structure with embedded edges. This type of induction argument will be repeated several times throughout this section.

To prove Theorem 6.1 we must also show that the 2-cells of $\Delta$ corresponding to $(n, m, k)$-triples are embedded and do not intersect one another. Since the 2cells are simply connected and totally geodesic, and since the $\mathcal{O}(n)$-edges in their boundary are geodesic arcs perpendicular to the horospheres at their endpoints, it is straightforward to show that if two 2-cells intersect, then an $m c O(n)$-edge in the boundary of one 2-cell must intersect the other 2-cell. The previous lemmas imply that this intersection must occur in the interior of the 2-cell. Therefore to complete the proof of Theorem 6.1 it is sufficient to prove the following:

Proposition 6.9. Suppose $N$ has a geometric Mom-n structure of one of the types described in Theorem 5.9. If any of the $\mathcal{O}(n)$-edges of the complex $\Delta$ intersect any of the 2-cells in $\Delta$, then there must exist a simpler geometric Mom-n structure in $N$, which is also of one of the types described in Theorem [5.9.

Proof. The idea of the proof is as follows: for every possible case, use the previous lemmas in this section to either demonstrate a contradiction or reduce the problem to a strictly previous case. But before enumerating the cases, consider the diagram in Figure 6, 


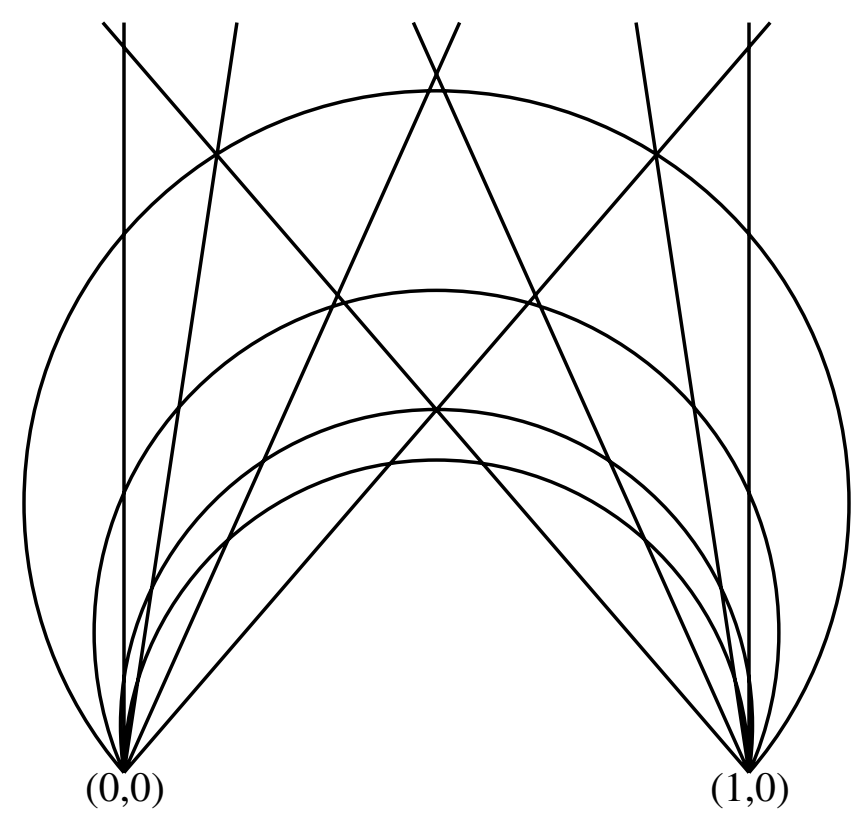

Figure 6. An ideal triangle, together with three equidistant curves for each side.

This diagram shows an ideal triangle in the upper half-space model of $\mathbb{H}^{2}$ with vertices at 0,1 , and $\infty$, together with nine curves that are each equidistant from one of the three sides. For each of the three sides of the triangle there are three curves: one at a distance of $\delta_{1}$ from the side, one at a distance of $\delta_{2}$, and one at a distance of $\delta_{3}$. (To construct this diagram it is sufficient to note the following. A curve in the upper half-space model which is at a constant distance $r$ from the line from 0 to $\infty$ is just a line passing through 0 with slope $\pm(\sinh r)^{-1}$; a curve which is equidistant from the line from 1 to $\infty$ is constructed similarly. A curve at constant distance $r$ from the line from 0 to 1 is a circular arc passing from 0 to 1 through the point $\left(\frac{1}{2}, \frac{1}{2} e^{ \pm r}\right)$. See for example [Thu].)

From the diagram, the following result is immediate:

Lemma 6.10. If $p$ is a point in the interior of the ideal triangle with vertices at 0,1 , and $\infty$, and if $\lambda_{1}, \lambda_{2}, \lambda_{3}$ are the sides of the triangle in any order, then:

(1) If $d\left(p, \lambda_{1}\right) \geq \delta_{3}$ and $d\left(p, \lambda_{2}\right) \geq \delta_{3}$, then $d\left(p, \lambda_{3}\right)<\delta_{1}$.

(2) If $d\left(p, \lambda_{1}\right) \geq \delta_{3}$ and $d\left(p, \lambda_{2}\right) \geq \delta_{1}$, then $d\left(p, \lambda_{3}\right)<\delta_{3}$.

(3) (Corollary to the previous part) If $d\left(p, \lambda_{1}\right) \geq \delta_{3}$, then either one of $d\left(p, \lambda_{2}\right)$, $d\left(p, \lambda_{3}\right)$ is $<\delta_{2}$ or else both of $d\left(p, \lambda_{2}\right), d\left(p, \lambda_{3}\right)$ are $<\delta_{3}$.

(4) If $d\left(p, \lambda_{i}\right) \geq \delta_{2}$ for all $i \in\{1,2,3\}$, then $d\left(p, \lambda_{j}\right)<\delta_{3}$ for at least two $j \in\{1,2,3\}$.

The conclusion in the third part of the above lemma is annoyingly weak. But note that the region in the ideal triangle where $d\left(p, \lambda_{1}\right) \geq \delta_{3}, d\left(p, \lambda_{2}\right) \geq \delta_{2}$, and $d\left(p, \lambda_{3}\right) \geq \delta_{2}$ is very small; if $\delta_{3}$ is replaced with even a slightly smaller number, then a stronger conclusion would result. This turns out to be useful enough that 


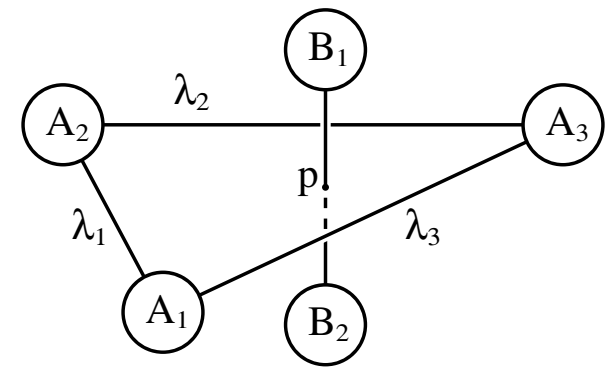

Figure 7. The horoballs and edges used in the proof of Proposition 6.9

we do so now; the proof of the following lemma, while not following immediately from the diagram, is elementary enough that we omit it for brevity:

Lemma 6.11. If $d\left(p, \lambda_{1}\right) \geq 0.9$, then one of $d\left(p, \lambda_{2}\right), d\left(p, \lambda_{3}\right)$ is $<\delta_{2}$.

Now we begin enumerating the various cases to prove Proposition 6.9. according to which edge is involved and which triple corresponds to the 2-cell involved. In each case, let $p$ be the point where the edge intersects the 2-cell, and note that the two-cell is contained in an ideal triangle which is isometric to that used in Lemma 6.10, We will refer to the sides of the triangle as $\lambda_{j}$ for $j \in\{1,2,3\}$ and let $d_{j}=d\left(p, \lambda_{j}\right)$. Also we will let $A_{1}, A_{2}$, and $A_{3}$ denote the horoballs such that $\lambda_{1}$ goes from $A_{1}$ to $A_{2}, \lambda_{2}$ goes from $A_{2}$ to $A_{3}$, and $\lambda_{3}$ goes from $A_{3}$ to $A_{1}$. Finally let $B_{1}$ and $B_{2}$ be the horoballs at the ends of the $\mathcal{O}(n)$-edge which passes through p. See Figure 7 .

Case $1\left(\mathcal{O}(1)\right.$-edge, any triple). Part 1 of Lemma6.10 implies that $d_{j}<\delta_{3}$ for some $j$. Now apply Lemma 6.6 to obtain a contradiction.

Case $2\left(\mathcal{O}(2)\right.$-edge, $(1,1,2)$-triple). Suppose that $\lambda_{1}$ and $\lambda_{2}$ contain lifts of the $\mathcal{O}(1)$-edge while $\lambda_{3}$ contains a lift of the $\mathcal{O}(2)$-edge. Part 1 of Lemma 6.10 implies that either $d_{1}<\delta_{3}, d_{2}<\delta_{3}$, or $d_{3}<\delta_{1}$. In the first two cases, Lemma 6.6 applies while in the third case, Lemma 6.8 applies. Either possibility leads to a contradiction.

Case $3(\mathcal{O}(2)$-edge, $(1,2,2)$-triple $)$. Suppose that $\lambda_{1}$ contains a lift of the $\mathcal{O}(1)$-edge while $\lambda_{2}$ and $\lambda_{3}$ contain lifts of the $\mathcal{O}(2)$-edge. If $d_{1}<\delta_{3}$, then Lemma 6.6 applies and we're done. If either $d_{2}$ or $d_{3}$ is less than $\delta_{2}$, then the same argument used in the proof of Lemma 6.7 proves the existence of a geometric Mom-2 structure involving only $(1,1,2)$-triples; either this new Mom-2 is embedded or else we reduce to case 2 .

By part 3 of Lemma 6.10 the only remaining possibility is that $d_{1} \geq \delta_{3}, d_{2}<\delta_{3}$, and $d_{3}<\delta_{3}$. Since $d_{2}<\delta_{3}$, by Lemma 6.5, at least three of the four horoball pairs $\left\{A_{2}, B_{1}\right\},\left\{B_{1}, A_{3}\right\},\left\{A_{3}, B_{2}\right\},\left\{B_{2}, A_{2}\right\}$ are elements of $\mathcal{O}(1)$. Similarly, since $d_{3}<\delta_{3}$ at least three of the four pairs $\left\{A_{3}, B_{1}\right\},\left\{B_{1}, A_{1}\right\},\left\{A_{1}, B_{2}\right\}$, and $\left\{B_{2}, A_{3}\right\}$ lie in $\mathcal{O}(1)$. But $\left\{A_{1}, A_{2}\right\}$ is already in $\mathcal{O}(1)$; hence by Corollary 3.3 at least one of $\left\{A_{1}, B_{1}\right\},\left\{A_{2}, B_{1}\right\}$ must not lie in $\mathcal{O}(1)$, and similarly one of $\left\{A_{1}, B_{2}\right\},\left\{A_{2}, B_{2}\right\}$ must not lie in $\mathcal{O}(1)$. Up to symmetry, we must have $\left\{A_{1}, B_{1}\right\},\left\{A_{2}, B_{2}\right\} \in \mathcal{O}(1)$, 
and $\left\{A_{2}, B_{1}\right\},\left\{A_{1}, B_{2}\right\} \notin \mathcal{O}(1)$. Hence $d\left(A_{2}, B_{1}\right) \geq o(2)$ and $d\left(A_{1}, B_{2}\right) \geq o(2)$. Now apply Corollary 3.6 to $A_{1}, A_{2}, B_{1}$, and $B_{2}$ to obtain

$$
\begin{aligned}
e_{2}^{2}+1 & \leq e_{2} \cosh d_{1} \\
\Rightarrow d_{1} & \geq \cosh ^{-1}\left(e_{2}+e_{2}{ }^{-1}\right) \\
& \geq \cosh ^{-1} 2 .
\end{aligned}
$$

Note that $\cosh ^{-1} 2=1.3169 \ldots$, so Lemma 6.11 applies. Hence one of $d_{2}, d_{3}$ is less than $\delta_{2}$, and a preceding argument applies. This completes the proof in this case.

We can save a bit of time at this point by noting that the argument in case 3 still works if we replace the $(1,2,2)$-triple with a $(1,2,3)$-triple, a $(1,3,3)$-triple, or (with minor modifications) a (1,1,3)-triple; henceforth we will assume that case 3 encompasses all of these possibilities.

Case $4\left(\mathcal{O}(3)\right.$-edge, $(1,1,2)$-triple). Suppose that $\lambda_{1}$ and $\lambda_{2}$ contain lifts of the $\mathcal{O}(2)$-edge while $\lambda_{3}$ contains a lift of the $\mathcal{O}(2)$-edge. If $d_{1}<\delta_{3}$ or $d_{2}<\delta_{3}$, then Lemma 6.6 applies, giving a contradiction. Otherwise by part 1 of Lemma 6.10 we have $d_{3}<\delta_{1}$. Since $\delta_{1}<\delta_{2}$, we can use the argument from Lemma 6.7 to find a geometric Mom-2 structure using only $(1,1,2)$-triples, reducing the problem to case 2 .

Case $5(\mathcal{O}(3)$-edge, $(1,1,3)$-triple $)$. This case is nearly identical to the previous one, except we use Lemma 6.8 to obtain a contradiction instead of using Lemma 6.7 to reduce to case 2 .

Case $6(\mathcal{O}(3)$-edge, $(1,2,2)$-triple $)$. Use the argument in case 3 with trivial modifications.

Case $7\left(\mathcal{O}(2)\right.$-edge, (2,2,3)-triple). Suppose that $\lambda_{1}$ and $\lambda_{2}$ contain lifts of the $\mathcal{O}(2)$-edge while $\lambda_{3}$ contains a lift of the $\mathcal{O}(3)$-edge. If $d_{i}<\delta_{2}$ for any $i \in\{1,2,3\}$, then we can use the argument from Lemma 6.7 as before to construct a geometric Mom-2 structure with just $(1,1,2)$-triples and reduce to case 2. So suppose $d_{i} \geq \delta_{2}$ for all $i \in\{1,2,3\}$; then by part 4 of Lemma 6.10 we have $d_{j}<\delta_{3}$ for at least two $j \in\{1,2,3\}$. Suppose that $d_{1}<\delta_{3}$ and $d_{2}<\delta_{3}$; the other possibilities can be handled similarly. Then by Lemma 6.5 at least three of the pairs $\left\{A_{1}, B_{1}\right\},\left\{B_{1}, A_{2}\right\},\left\{A_{2}, B_{2}\right\}$, and $\left\{B_{2}, A_{1}\right\}$ lie in $\mathcal{O}(1)$, and at least three of the pairs $\left\{A_{2}, B_{1}\right\},\left\{B_{1}, A_{3}\right\},\left\{A_{3}, B_{2}\right\}$, and $\left\{B_{2}, A_{2}\right\}$ lie in $\mathcal{O}(1)$.

Suppose all three of the pairs $\left\{A_{1}, B_{i}\right\},\left\{A_{2}, B_{i}\right\}$, and $\left\{A_{3}, B_{i}\right\}$ were in $\mathcal{O}(1)$ for some $i \in\{1,2\}$. Then both of the triples $\left\{A_{1}, A_{2}, B_{i}\right\}$ and $\left\{A_{2}, A_{3}, B_{i}\right\}$ are $(1,1,2)$-triples. If there is an isometry $g \in \pi_{1}(N)$ taking one triple to another, then that isometry must be a parabolic isometry fixing $B_{i}$ and taking $A_{1}$ to $A_{2}$ and $A_{2}$ to $A_{3}$. But this would imply that the arc from $A_{1}$ to $A_{3}$ intersects either $B_{i}, A_{2}$, or the arc from $B_{i}$ to $A_{2}$, contradicting our assumptions to this point. If there is no such isometry $g$, then we have a geometric Mom-2 structure with two distinct $(1,1,2)$-triples and can reduce to case 2.

So suppose that at least one of the pairs $\left\{A_{1}, B_{i}\right\},\left\{A_{2}, B_{i}\right\}$, and $\left\{A_{3}, B_{i}\right\}$ does not lie in $\mathcal{O}(1)$, both for $i=1$ and for $i=2$. Up to symmetry, the only way this can happen given our earlier assumptions is if the pairs $\left\{A_{1}, B_{1}\right\},\left\{B_{1}, A_{2}\right\},\left\{A_{2}, B_{2}\right\}$, and $\left\{B_{2}, A_{3}\right\}$ lie in $\mathcal{O}(1)$ while the pairs $\left\{A_{1}, B_{2}\right\}$ and $\left\{A_{3}, B_{1}\right\}$ do not. If $\left\{A_{1}, B_{2}\right\}$ 
lies in $\mathcal{O}(2)$, then the triples $\left\{A_{1}, A_{2}, B_{2}\right\}$ and $\left\{B_{1}, B_{2}, A_{2}\right\}$ form a geometric Mom2 structure with one $(1,1,2)$-triple and one $(1,2,2)$-triple, allowing us to reduce the problem to either case 2 or case 3 . So suppose $d\left(A_{1}, B_{2}\right) \geq o(3)$. For similar reasons we can suppose that $d\left(A_{3}, B_{1}\right) \geq o(3)$. Then applying Corollary 3.6 to $A_{1}$, $A_{3}, B_{1}$, and $B_{2}$ we obtain

$$
\begin{aligned}
e_{3}{ }^{2}+1 & \leq e_{2} e_{3} \cosh d_{3} \\
& \leq e_{3}^{2} \cosh d_{3} \\
\Rightarrow d_{3} & \geq \cosh ^{-1}\left(1+e_{3}^{-2}\right) .
\end{aligned}
$$

Note that if $e_{3} \leq 1.5152$, then this implies that $d_{3} \geq 0.9$ and hence Lemma 6.11 applies. Therefore at least one of $d_{1}, d_{2}$ is less than $\delta_{2}$, and a preceding argument applies. This completes the proof in this case.

Case $8(\mathcal{O}(2)$-edge,(2,3,3)-triple). An almost identical argument to the one in case 7 applies, except that the problem may reduce to case 5 instead of case 2 .

Case $9\left(\mathcal{O}(3)\right.$-edge,(2,2,3)-triple). Suppose that $\lambda_{1}$ and $\lambda_{2}$ contain lifts of the $\mathcal{O}(2)$ edge while $\lambda_{3}$ contains a lift of the $\mathcal{O}(3)$-edge. If $d_{3}<\delta_{1}$, then Lemma 6.8 provides a contradiction, while if $d_{i}<\delta_{2}$ for $i=1$ or 2 , then the argument from Lemma 6.7 will produce a simpler geometric Mom-2 structure. Hence suppose that $d_{3} \geq \delta_{1}$ and $d_{i} \geq \delta_{2}>\delta_{1}$ for $i=1$ and 2. Part 2 of Lemma 6.10 then implies that $d_{j}<\delta_{3}$ for at least two different $j \in\{1,2,3\}$.

Suppose $d_{1}<\delta_{3}$ and $d_{3}<\delta_{3}$. Since $d_{1}<\delta_{3}$, by Lemma 6.5 at least three of $\left\{A_{1}, B_{1}\right\},\left\{B_{1}, A_{2}\right\},\left\{A_{2}, B_{2}\right\},\left\{B_{2}, A_{1}\right\}$ lie in $\mathcal{O}(1)$. Without loss of generality assume that $\left\{A_{1}, B_{1}\right\}$ and $\left\{B_{1}, A_{2}\right\}$ lie in $\mathcal{O}(1)$. If $\left\{B_{1}, A_{3}\right\}$ lies in $\mathcal{O}(1)$, then the triples $\left\{A_{1}, B_{1}, A_{2}\right\}$ and $\left\{A_{2}, B_{1}, A_{3}\right\}$ are both $(1,1,2)$-triples, and they form a simpler geometric Mom-2 structure unless they are equivalent by the action of a parabolic element $g \in \pi_{1}(N)$ which fixes $B_{1}$; but this would imply that the arc from $B_{1}$ to $A_{3}$ intersects the arc from $A_{1}$ to $A_{2}$, a simpler case. If $\left\{B_{1}, A_{3}\right\}$ lies in $\mathcal{O}(2)$, then $\left\{A_{1}, B_{1}, A_{2}\right\}$ is a $(1,1,2)$-triple while $\left\{A_{2}, B_{1}, A_{3}\right\}$ is a $(1,2,2)$-triple and we have a simpler Mom-2. So suppose $\left\{B_{1}, A_{3}\right\} \notin \mathcal{O}(1) \cup \mathcal{O}(2)$. Then since $d_{3}<\delta_{3}$, by Lemma 6.5 the pairs $\left\{B_{2}, A_{1}\right\}$ and $\left\{B_{2}, A_{3}\right\}$ must lie in $\mathcal{O}(1) \cup \mathcal{O}(2)$. Now consider $\left\{B_{2}, A_{2}\right\}$; if this pair lies in $\mathcal{O}(1) \cup \mathcal{O}(2)$, then the triples $\left\{A_{1}, A_{2}, B_{1}\right\}$ and $\left\{A_{1}, A_{2}, B_{2}\right\}$ will form a simpler geometric Mom-2 structure, so suppose $\left\{B_{2}, A_{2}\right\} \notin$ $\mathcal{O}(1) \cup \mathcal{O}(2)$. We have reached a point where $d\left(B_{1}, A_{3}\right) \geq o(3)$ and $d\left(B_{2}, A_{2}\right) \geq o(3)$, so applying Corollary 3.6 to the four horoballs $A_{2}, A_{3}, B_{1}, B_{3}$ we get

$$
\begin{aligned}
e_{3}{ }^{2}+1 & \leq e_{2} e_{3} \cosh d_{2} \\
& \leq e_{3}^{2} \cosh d_{2} \\
\Rightarrow d_{2} & \geq \cosh ^{-1}\left(1+e_{3}{ }^{-2}\right) .
\end{aligned}
$$

Note that if $e_{3} \leq 1.5152$, then this implies that $d_{2} \geq 0.9$ and hence Lemma 6.11 implies that at least one of $d_{1}, d_{3}$ is less than $\delta_{2}$. But if $d_{1}<\delta_{2}$, then by Corollary 3.6 we would have $\left\{B_{2}, A_{2}\right\} \in \mathcal{O}(1)$, while if $d_{3}<\delta_{2}$, then $\left\{B_{1}, A_{3}\right\} \in \mathcal{O}(1) \cup \mathcal{O}(2)$; both conclusions contradict our assumptions to this point.

The argument when $d_{2}<\delta_{3}$ and $d_{3}<\delta_{3}$ is identical to this one by symmetry, and the argument when $d_{1}<\delta_{3}$ and $d_{2}<\delta_{3}$ is similar.

Case $10(\mathcal{O}(3)$-edge, $(1,2,3)$-triple). (This is by far the trickiest case.) Suppose that $\lambda_{1}$ contains a lift of the $\mathcal{O}(1)$-edge, $\lambda_{2}$ contains a lift of the $\mathcal{O}(2)$-edge, and $\lambda_{3}$ 
contains a lift of the $\mathcal{O}(3)$-edge. If $d_{1}<\delta_{3}$, then Lemma 6.6 leads to a contradiction. If $d_{2}<\delta_{2}$, then as before we can repeat the argument of Lemma 6.7 to obtain a simpler Mom-2 and reduce to case 2. While if $d_{3}<\delta_{1}$, then Lemma 6.8 provides a contradiction.

So suppose that $d_{1} \geq \delta_{3}, d_{2} \geq \delta_{2}$, and $d_{3} \geq \delta_{1}$. Note that by part 2 of Lemma 6.10 this implies that $d_{2}<\delta_{3}$ and $d_{3}<\delta_{3}$. By Lemma 6.5] at least three of the four pairs $\left\{A_{2}, B_{1}\right\},\left\{B_{1}, A_{3}\right\},\left\{A_{3}, B_{2}\right\}$, and $\left\{B_{2}, A_{1}\right\}$ lie in $\mathcal{O}(1)$, while at least three of the four pairs $\left\{A_{1}, B_{1}\right\},\left\{B_{1}, A_{3}\right\},\left\{A_{3}, B_{2}\right\},\left\{B_{2}, A_{1}\right\}$ lie in $\mathcal{O}(1) \cup \mathcal{O}(2)$. Unfortunately this is not quite enough information to construct a simpler Mom-2 or Mom-3, so we must dig deeper.

Let $\sigma$ be the 2-cell spanning $A_{1}, A_{2}, A_{3}$, and the arcs between them. Since $\left\{A_{3}, A_{1}\right\}$ and $\left\{B_{1}, B_{2}\right\}$ are both elements of the orthopair class $\mathcal{O}(3)$, there exists a group element $g \in \pi_{1}(N)$ which sends $\left\{A_{3}, A_{1}\right\}$ to $\left\{B_{1}, B_{2}\right\}$. Furthermore since $\sigma$ is totally geodesic, $g(\sigma) \cap \sigma$ must contain a geodesic line segment with one endpoint $p$ in the arc from $B_{1}$ to $B_{2}$. Let $q$ be the other endpoint of this line segment. What are the possible locations of $q$ ?

If $q$ lies in the interior of $\sigma$, then either $g\left(A_{j}\right)$ intersects $\sigma$ for some $j$, in which case we can apply Lemma 6.3. or else either $g\left(\lambda_{1}\right)$ or $g\left(\lambda_{2}\right)$ intersect $\sigma$, in which case the problem reduces to case 1 or case 3 , respectively. So suppose $q$ lies on the boundary of $\sigma$. If $q$ lies in the interior of $\lambda_{1}$, then $g^{-1}\left(\lambda_{1}\right)$ intersects $\sigma$ and we can reduce to case 1 . Similarly if $q$ lies in the interior of $\lambda_{2}$, then we can reduce to case 3.

If $q$ lies in $\sigma \cap A_{2}$, then $g$ must be a parabolic element of $\pi_{1}(N)$ fixing $A_{2}$. But note that the projections of $\lambda_{3}$ and $g\left(\lambda_{3}\right)$ onto the surface of $A_{2}$ clearly intersect; if $g$ is parabolic fixing $A_{2}$, then the only way this can happen is if $\lambda_{3}$ and $g\left(\lambda_{3}\right)$ themselves intersect. This contradicts Lemma 6.8.

If $q$ lies in $\sigma \cap A_{3}$, then $g$ must send the triple $\left\{A_{1}, A_{2}, A_{3}\right\}$ to the triple $\left\{A_{3}, B_{1}, B_{2}\right\}$; in particular $\left\{A_{3}, B_{1}, B_{2}\right\}$ is a $(1,2,3)$-triple. Without loss of generality, assume that $\left\{A_{3}, B_{2}\right\}$ lies in $\mathcal{O}(2)$ and $\left\{A_{3}, B_{1}\right\}$ lies in $\mathcal{O}(1)$. Since $d_{2} \leq \delta_{3}$, this implies that $\left\{A_{2}, B_{1}\right\}$ and $\left\{A_{2}, B_{2}\right\}$ lie in $\mathcal{O}(1)$ as well. Thus $\left\{A_{2}, B_{1}, A_{3}\right\}$ is a $(1,1,2)$-triple while $\left\{A_{2}, B_{2}, A_{3}\right\}$ is a $(1,2,2)$-triple, forming a simpler geometric Mom-2 structure and reducing the problem to either case 1,2 , or 3.

There are two remaining possibilities. Suppose $q$ lies in $\sigma \cap A_{1}$; then $g$ must send $\left\{A_{1}, A_{2}, A_{3}\right\}$ to $\left\{A_{1}, B_{1}, B_{2}\right\}$. Note in particular that we must have $g\left(A_{2}\right)=A_{1}$. Without loss of generality assume that $\left\{A_{1}, B_{1}\right\}$ lies in $\mathcal{O}(1)$ while $\left\{A_{1}, B_{2}\right\}$ lies in $\mathcal{O}(2)$. Applying Lemma 3.2 to the triple $\left\{A_{1}, A_{2}, B_{1}\right\}$ we see that $\left\{A_{2}, B_{1}\right\}$ cannot be in $\mathcal{O}(1)$. Since $d_{2}<\delta_{3}$, we must have all of $\left\{B_{1}, A_{3}\right\},\left\{A_{3}, B_{2}\right\},\left\{B_{2}, A_{2}\right\}$ in $\mathcal{O}(1)$ instead. So $\left\{A_{1}, B_{1}, A_{3}\right\}$ is a $(1,1,3)$-triple, while $\left\{A_{1}, B_{2}, A_{3}\right\}$ is a $(1,2,3)$-triple. Now consider $g^{-1}(\sigma)$. Comparing $\sigma$ and $g(\sigma)$, we see that $g^{-1}(\sigma)$ must intersect $\lambda_{3}$, and must be bounded at one corner by $A_{2}=g^{-1}\left(A_{1}\right)$. Specifically $g^{-1}\left(\lambda_{1}\right) \in \mathcal{O}(1)$ and $g^{-1}\left(\lambda_{3}\right) \in \mathcal{O}(3)$ must have one endpoint on $A_{2}$. If any of the four arcs spanning the horoball pairs $\left\{A_{1}, B_{1}\right\},\left\{B_{1}, A_{3}\right\},\left\{A_{3}, B_{2}\right\}$, or $\left\{B_{2}, A_{1}\right\}$ (all of which are in $\mathcal{O}(1) \cup \mathcal{O}(2))$ intersect $g^{-1}(\sigma)$, then we can reduce to a previous case, so suppose this doesn't happen. We still must have $g^{-1}(\sigma)$ intersecting $\lambda_{3}$. If either $g^{-1}\left(\lambda_{1}\right)$ or $g^{-1}\left(\lambda_{2}\right)$ intersect either of the two-cells spanning the triples $\left\{A_{1}, B_{1}, A_{3}\right\}$ or $\left\{A_{1}, B_{2}, A_{3}\right\}$, then we can still reduce the problem to a previous case. The only way $g^{-1}(\sigma)$ can intersect $\lambda_{3}$ without such an intersection occurring (and without $g^{-1}(\sigma)$ intersecting the interior of a horoball, which contradicts Lemma 6.3) is if 
$g^{-1}\left(A_{1}\right)=B_{2}$ and $g^{-1}\left(\lambda_{3}\right)$ intersects the two-cell spanning $\left\{A_{1}, B_{1}, A_{3}\right\}$. But this merely lets us reduce to case 5 instead.

Thus we come to the final possibility: suppose that $q$ lies in the interior of $\lambda_{3}$. Then the image of the arc from $p$ to $q$ under $g^{-1}$ must be another geodesic line segment going from $g^{-1}(p)$ on $\lambda_{3}$ to $g^{-1}(q)$ in the interior of $\sigma$. Note that $p \neq g^{-1}(q)$; otherwise, $g$ would be elliptic of order 2. Since $g^{-1}(q)$ is the point where $\sigma$ intersects the arc from $g^{-1}\left(A_{1}\right)$ to $g^{-1}\left(A_{3}\right)$, and since this arc is also a lift of the $\mathcal{O}(3)$-edge, the same arguments that apply to $p$ also apply to $g^{-1}(q)$. Specifically: if $d\left(g^{-1}(q), \lambda_{1}\right)<\delta_{3}, d\left(g^{-1}(q), \lambda_{2}\right)<\delta_{2}$, or $d\left(g^{-1}(q), \lambda_{3}\right)<\delta_{1}$, then we can apply Lemmas 6.6, 6.7, or 6.8 respectively to get either a contradiction or a reduction to a simpler case. So assume that none of those three inequalities holds. Note that this implies that $d\left(g^{-1}(q), \lambda_{3}\right)<\delta_{3}$ just as our previous assumptions implied that $d_{3}=d\left(p, \lambda_{3}\right)<\delta_{3}$.

Now consider the pairs $\left\{B_{1}, B_{2}\right\}$ and $\left\{g^{-1}\left(A_{1}\right), g^{-1}\left(A_{3}\right)\right\}$. These cannot be the same pair; if they were, then $g^{2}$ would fix the pair $\left\{B_{1}, B_{2}\right\}$ and hence be either elliptic or the identity, a contradiction. So the two pairs are either completely disjoint or else intersect in a single element. Suppose they intersect in a single element; without loss of generality, assume in particular that $B_{1}=g^{-1}\left(A_{1}\right)$. Note that we can't have $g\left(A_{1}\right)=B_{1}$; if we did, then $g^{2}$ would fix $A_{1}$ and hence so would $g$, a contradiction. So $g\left(A_{1}\right)=B_{2}$, and $g\left(A_{3}\right)=B_{1}$. To summarize we have $g\left(B_{1}\right)=A_{1}$ and $g^{2}\left(B_{1}\right)=B_{2}$, while at the same time we must have $g^{-1}\left(B_{1}\right)=A_{3}$. Arrange the upper half-space model of $\mathbb{H}^{3}$ so that $B_{1}$ is centered at the point at infinity, $A_{1}=g\left(B_{1}\right)$ and $A_{3}=g^{-1}\left(B_{1}\right)$ are centered on the real line, and $A_{2}$ is centered at some point with positive imaginary part. By assumption, the arcs from $B_{1}$ to $B_{2}$ and from $B_{1}$ to $g^{-1}\left(A_{3}\right)$ both intersect the interior of the two-cell spanning the triple $\left\{A_{1}, A_{2}, A_{3}\right\}$; hence the centers of $B_{2}=g^{2}\left(B_{1}\right)$ and $g^{-1}\left(A_{3}\right)=g^{-2}\left(B_{1}\right)$ also have positive imaginary part. But this is impossible: by direct calculation in $\operatorname{PSL}(2, \mathbb{C})$, if $g$ is an element such that $g(\infty)$ and $g^{-1}(\infty)$ are both real, then either $g^{2}(\infty)$ and $g^{-2}(\infty)$ are also both real or else their imaginary parts have opposite signs. Hence, the pairs $\left\{B_{1}, B_{2}\right\}$ and $\left\{g^{-1}\left(A_{1}\right), g^{-1}\left(A_{3}\right)\right\}$ must be completely disjoint, or in other words, the arcs $g\left(\lambda_{3}\right)$ and $g^{-1}\left(\lambda_{3}\right)$ do not share a horoball at their endpoints.

Now suppose that $d\left(\lambda_{3}, g\left(\lambda_{3}\right)\right) \geq \delta_{2}$ and $d\left(\lambda_{3}, g^{-1}\left(\lambda_{3}\right)\right) \geq \delta_{2}$. Examining Figure 66 (and remembering our previous assumptions), this implies that both $p$ and $g^{-1}(q)$ must lie in the small region bounded by the curves $d\left(\cdot, \lambda_{1}\right)=\delta_{3}, d\left(\cdot, \lambda_{2}\right)=\delta_{2}$, and $d\left(\cdot, \lambda_{3}\right)=\delta_{2}$. The diameter of this region can be readily computed (it's not a triangle but it is contained inside one), and it is far less than $\delta_{1}$. Hence $d\left(g\left(\lambda_{3}\right), g^{-1}\left(\lambda_{3}\right)\right)<\delta_{1}$, and hence Lemma 6.8 applies, producing a contradiction. So therefore one of $d\left(\lambda_{3}, g\left(\lambda_{3}\right)\right)$ and $d\left(\lambda_{3}, g^{-1}\left(\lambda_{3}\right)\right)$ must be less than $\delta_{2}$, and clearly if one is, then both are by isometry. Furthermore, the diameter of the region in Figure 6 bounded by the curves $d\left(\cdot, \lambda_{1}\right)=\delta_{3}, d\left(\cdot, \lambda_{2}\right)=\delta_{2}$, and $d\left(\cdot, \lambda_{3}\right)=\delta_{1}$ can also be computed to be less than $\delta_{3}$. Then by applying Lemma 6.5 repeatedly, we get the following:

- All four of the pairs $\left\{A_{1}, B_{1}\right\},\left\{B_{1}, A_{3}\right\},\left\{A_{3}, B_{2}\right\}$, and $\left\{B_{2}, A_{1}\right\}$ lie in $\mathcal{O}(1) \cup \mathcal{O}(2)$.

- All four of the pairs $\left\{A_{1}, g^{-1}\left(A_{1}\right)\right\},\left\{g^{-1}\left(A_{1}\right), A_{3}\right\},\left\{A_{3}, g^{-1}\left(A_{3}\right)\right\}$, and $\left\{g^{-1}\left(A_{3}\right), A_{1}\right\}$ lie in $\mathcal{O}(1) \cup \mathcal{O}(2)$. 


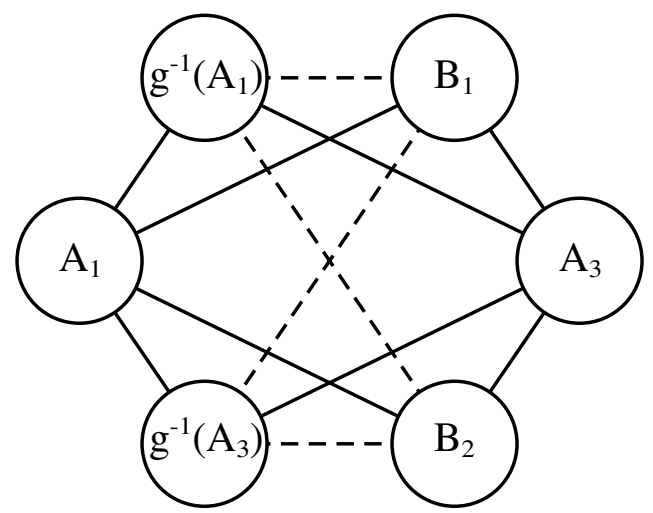

Figure 8. A possible collection of horoballs used in case 10; the solid lines (and three of the four dashed lines) indicate pairs which lie in $\mathcal{O}(1) \cup \mathcal{O}(2)$.

- At least three of the pairs $\left\{B_{1}, g^{-1}\left(A_{1}\right)\right\},\left\{g^{-1}\left(A_{1}\right), B_{2}\right\},\left\{B_{2}, g^{-1}\left(A_{3}\right)\right\}$, and $\left\{g^{-1}\left(A_{3}\right), B_{1}\right\}$ lie in $\mathcal{O}(1) \cup \mathcal{O}(2)$.

So the six horoballs $A_{1}, A_{3}, B_{1}, B_{2}, g^{-1}\left(A_{1}\right)$, and $g^{-1}\left(A_{3}\right)$ form a picture such as the one in Figure 8, where each of the solid edges and three of the four dashed edges are a lift of either the $\mathcal{O}(1)$-edge or the $\mathcal{O}(2)$-edge. Suppose, for the sake of an example, that the dashed edge from $B_{1}$ to $g^{-1}\left(A_{1}\right)$ is the only edge which is not a lift of either the $\mathcal{O}(1)$-edge or the $\mathcal{O}(2)$-edge. Then consider the triple $\left\{A_{1}, B_{2}, g^{-1}\left(A_{3}\right)\right\}$. This triple must be an $(a, b, b)$-triple, where $a$ and $b$ are 1 and 2 in some order (i.e., it's either a $(1,1,2)$-triple or a $(1,2,2)$-triple). But this triple also has the property that each edge of the triple is shared by another triple in the diagram which must also be either a $(1,1,2)$-triple or a $(1,2,2)$-triple. It is straightforward to see that some pair of these four triples, specifically the pair which shares the edge which is a lift of the $\mathcal{O}(a)$-edge, must be distinct under the action of $\pi_{1}(N)$ and hence form a geometric Mom-2 structure. A similar argument holds for each of the other dashed edges in the diagram.

This at last completes the proof in this case.

Case $11\left(\mathcal{O}(3)\right.$-edge,(1,3,3)-triple). Suppose $\lambda_{3}$ contains a lift of the $\mathcal{O}(1)$-edge while $\lambda_{1}$ and $\lambda_{2}$ contain lifts of the $\mathcal{O}(3)$-edge. If $d_{3}<\delta_{3}$, then Lemma 6.6 provides a contradiction, while if $d_{i}<\delta_{1}$ for $i=1$ or 2, then Lemma 6.8 provides a contradiction. So suppose $d_{1} \geq \delta_{1}, d_{2} \geq \delta_{1}$, and $d_{3} \geq \delta_{3}$. Lemma 6.10 implies that $d_{1}$ and $d_{2}$ are both less than $\delta_{3}$.

Suppose that in fact $d_{1}<\delta_{2}$. By Lemma 6.5 all four pairs $\left\{A_{1}, B_{1}\right\},\left\{B_{1}, A_{2}\right\}$, $\left\{A_{2}, B_{2}\right\},\left\{B_{2}, A_{1}\right\}$ and at least three of the four pairs $\left\{A_{2}, B_{1}\right\},\left\{B_{1}, A_{3}\right\},\left\{A_{3}, B_{2}\right\}$, $\left\{B_{2}, A_{2}\right\}$ lie in $\mathcal{O}(1) \cup \mathcal{O}(2)$. Without loss of generality, assume that $\left\{B_{1}, A_{3}\right\}$ $\in \mathcal{O}(1) \cup \mathcal{O}(2)$. We wish to construct a geometric Mom-2 or Mom-3 structure that only uses triples considered in the previous cases. However we have to be careful to ensure that we do not construct a Mom-3 which is not torus-friendly; this can be ensured by not selecting any geometric Mom-3 structure that contains exactly two $(1,2,3)$-triples. To start with, note that the triple $\left\{A_{2}, B_{1}, A_{3}\right\}$ is of type $(1,1,2)$ 
or $(1,2,2)$. Now consider the four triples $\left\{A_{1}, B_{1}, A_{2}\right\},\left\{A_{1}, B_{2}, A_{2}\right\},\left\{B_{1}, A_{1}, B_{2}\right\}$, and $\left\{B_{1}, A_{2}, B_{2}\right\}$. Each of these triples is of type $(1,2,3),(1,1,3)$, or $(2,2,3)$. If all four of these triples are of type $(1,2,3)$, then no two triples can be equivalent under the action of $\pi_{1}(N)$ because each two of those triples share a common "edge". So any three of those triples will form a geometric Mom-3 structure which is torusfriendly. If between one and three of these triples are of type $(1,2,3)$, choose one triple of type $(1,2,3)$, one triple not of type $(1,2,3)$, and $\left\{A_{2}, B_{1}, A_{3}\right\}$ to get a simpler geometric Mom-3 structure which is torus-friendly. Also, if none of the four triples is of type $(1,2,3)$, then all four must be of type $(a, a, 3)$ for a fixed $a \in\{1,2\}$. Pick two such triples, say $\left\{A_{1}, B_{1}, A_{2}\right\}$ and $\left\{A_{1}, B_{2}, A_{2}\right\}$; these triples can't be equivalent under the action of $\pi_{1}(N)$ since that group has no elliptic elements; hence they form a simpler geometric Mom-2 structure.

So instead suppose that $d_{1} \geq \delta_{2}$ and for similar reasons suppose that $d_{2} \geq \delta_{2}$. Suppose now that $\left\{A_{2}, B_{1}\right\} \notin \mathcal{O}(1) \cup \mathcal{O}(2)$. Since $d_{1}$ and $d_{2}$ are both less than $\delta_{3}$, Lemma 6.5 implies that all of $\left\{A_{1}, B_{1}\right\},\left\{A_{1}, B_{2}\right\},\left\{A_{3}, B_{1}\right\},\left\{A_{3}, B_{2}\right\}$, and $\left\{A_{2}, B_{2}\right\}$ are in $\mathcal{O}(1) \cup \mathcal{O}(2)$. Then the triples $\left\{A_{1}, B_{1}, A_{3}\right\}$ and $\left\{A_{1}, B_{2}, A_{3}\right\}$ are each of type $(1,1,2)$ or $(1,2,2)$, and the triples $\left\{A_{1}, B_{2}, A_{2}\right\}$ and $\left\{A_{2}, B_{2}, A_{3}\right\}$ are each of type $(1,1,3),(1,2,3)$, or $(2,2,3)$. If $\left\{A_{1}, B_{2}, A_{2}\right\}$ and $\left\{A_{2}, B_{2}, A_{3}\right\}$ are equivalent due to the action of $g \in \pi_{1}(N)$, then $g$ must be parabolic fixing $B_{2}$, and hence $\lambda_{3}$ must intersect the arc from $B_{2}$ to $A_{2}$, a previous case. So $\left\{A_{1}, B_{2}, A_{2}\right\},\left\{A_{2}, B_{2}, A_{3}\right\}$, and $\left\{A_{1}, B_{2}, A_{3}\right\}$ are all distinct triples and form a simpler Mom-3 which furthermore is torus-friendly, unless $\left\{A_{1}, B_{2}, A_{2}\right\}$ and $\left\{A_{2}, B_{2}, A_{3}\right\}$ are both of type $(1,2,3)$. That is only possible if $\left\{A_{1}, B_{2}\right\}$ and $\left\{B_{2}, A_{3}\right\}$ are both in $\mathcal{O}(2)$ (the other possibility, that they are both in $\mathcal{O}(1)$, makes $\left\{A_{1}, B_{2}, A_{3}\right\}$ a $(1,1,1)$-triple, which is impossible). In this case $\left\{A_{1}, B_{2}, A_{3}\right\}$ is of type $(1,2,2)$ and cannot be equivalent to $\left\{A_{1}, B_{1}, A_{3}\right\}$ under the action of $\pi_{1}(N)$ since a group element sending one triple to another would have to fix the pair $\left\{A_{1}, A_{3}\right\}$; thus we have a simpler geometric Mom-2 structure.

So we may assume that $\left\{A_{2}, B_{1}\right\} \in \mathcal{O}(1) \cup \mathcal{O}(2)$, and by symmetry we may assume that $\left\{A_{2}, B_{2}\right\} \in \mathcal{O}(1) \cup \mathcal{O}(2)$. If the same holds for both $\left\{A_{1}, B_{1}\right\}$ and $\left\{A_{1}, B_{2}\right\}$, then we may proceed exactly as if $d_{1}<\delta_{2}$. So assume that one of those two pairs is not in $\mathcal{O}(1) \cup \mathcal{O}(2)$, and similarly assume that one of the pairs $\left\{A_{3}, B_{1}\right\}$, $\left\{A_{3}, B_{2}\right\}$ is not in $\mathcal{O}(1) \cup \mathcal{O}(2)$. Now applying Corollary 3.6 to the four horoballs $A_{1}, A_{3}, B_{1}$, and $B_{3}$, we get either

$$
e_{3}^{2}+1 \leq e_{3} \cosh d_{3}
$$

or

$$
2 e_{3} \leq e_{3} \cosh d_{3} \text {. }
$$

In either case, $d_{3} \geq \cosh ^{-1} 2>0.9$, and hence by Lemma 6.11, one of $d_{1}, d_{2}$ must be less than $\delta_{2}$, contradicting our assumptions to this point and completing this case.

Case $12\left(\mathcal{O}(3)\right.$-edge, $(2,3,3)$-triple). Suppose $\lambda_{1}$ and $\lambda_{2}$ contain lifts of the $\mathcal{O}(3)$ edge while $\lambda_{3}$ contains a lift of the $\mathcal{O}(2)$-edge.

Note that if $d_{1}<\delta_{1}$ or $d_{2}<\delta_{1}$, then we get a contradiction from Lemma 6.8, and if $d_{3}<\delta_{2}$, then we can produce a simpler geometric Mom-2 structure just as in Lemma 6.7. So assume $d_{1} \geq \delta_{1}, d_{2} \geq \delta_{1}$, and $d_{3} \geq \delta_{2}>\delta_{1}$. By Lemma 6.10 this implies that at least two of $d_{1}, d_{2}$, and $d_{3}$ must be less than $\delta_{3}$, and by Lemma 6.5 that in turn implies that at least two of the following statements are true: 
- At least three of $\left\{A_{1}, B_{1}\right\},\left\{B_{1}, A_{2}\right\},\left\{A_{2}, B_{2}\right\},\left\{B_{2}, A_{1}\right\}$ are in $\mathcal{O}(1) \cup \mathcal{O}(2)$.

- At least three of $\left\{A_{2}, B_{1}\right\},\left\{B_{1}, A_{3}\right\},\left\{A_{3}, B_{2}\right\},\left\{B_{2}, A_{2}\right\}$ are in $\mathcal{O}(1) \cup \mathcal{O}(2)$.

- At least three of $\left\{A_{3}, B_{1}\right\},\left\{B_{1}, A_{1}\right\},\left\{A_{1}, B_{2}\right\},\left\{B_{2}, A_{3}\right\}$ are in $\mathcal{O}(1) \cup \mathcal{O}(2)$.

Suppose for a moment that all four of $\left\{A_{1}, B_{1}\right\},\left\{B_{1}, A_{3}\right\},\left\{A_{3}, B_{2}\right\},\left\{B_{2}, A_{1}\right\}$ are in $\mathcal{O}(1) \cup \mathcal{O}(2)$. I.e., suppose that $\left\{A_{1}, B_{1}, A_{3}\right\}$ and $\left\{A_{1}, B_{2}, A_{3}\right\}$ are both of type $(1,1,2)$ or $(1,2,2)$. If these two triples are not equivalent under $\pi_{1}(N)$, then they form a simpler geometric Mom-2 structure. If they are equivalent due to $g \in \pi_{1}(N)$, then either $g$ is elliptic and fixes $\left\{A_{1}, A_{3}\right\}$ (a contradiction), $g$ is parabolic fixing one of $A_{1}$ or $A_{3}$ (in which case the one-cell from $B_{1}$ to $B_{2}$ will intersect another one-cell, reducing the problem to a previous case), or else up to symmetry we may assume that $\left\{A_{1}, B_{1}\right\},\left\{A_{3}, B_{2}\right\}$ are in $\mathcal{O}(1)$ and $\left\{A_{1}, B_{2}\right\}$, $\left\{A_{3}, B_{1}\right\}$ are in $\mathcal{O}(2)$. In the latter case, assume without loss of generality that $\left\{B_{1}, A_{2}\right\} \in \mathcal{O}(1) \cup \mathcal{O}(2)$ (we know this must be true for one of $\left\{B_{1}, A_{2}\right\},\left\{B_{2}, A_{2}\right\}$ ). Then one of the two triples $\left\{A_{1}, A_{2}, B_{1}\right\}$ and $\left\{A_{2}, A_{3}, B_{1}\right\}$ must be of type $(2,2,3)$ or $(1,1,3)$; this triple along with $\left\{A_{1}, B_{1}, A_{2}\right\}$ and $\left\{B_{1}, B_{2}, A_{1}\right\}$ forms a simpler geometric Mom-3 structure where each triple is of a different type (and hence none are equivalent under $\pi_{1}(N)$ ) and exactly one triple is of type $(1,2,3)$ (so the Mom-3 is torus-friendly).

Hence we may assume that at most three of $\left\{A_{1}, B_{1}\right\},\left\{B_{1}, A_{3}\right\},\left\{A_{3}, B_{2}\right\}$, $\left\{B_{2}, A_{1}\right\}$ are in $\mathcal{O}(1) \cup \mathcal{O}(2)$.

If all four of $\left\{A_{1}, B_{1}\right\},\left\{B_{1}, A_{2}\right\},\left\{A_{2}, B_{2}\right\},\left\{B_{2}, A_{1}\right\}$ lie in $\mathcal{O}(1) \cup \mathcal{O}(2)$ (or by symmetry all four of $\left\{A_{2}, B_{1}\right\},\left\{B_{1}, A_{3}\right\},\left\{A_{3}, B_{2}\right\},\left\{B_{2}, A_{2}\right\}$ ), then by a similar argument to the one used in the beginning of the previous case we can also construct a simpler Mom-2 or a simpler geometric Mom-3 structure which is torus-friendly. So assume this does not happen either. In summary, we can assume that for none of the three sets of horoball pairs listed above do all four pairs in the set lie in $\mathcal{O}(1) \cup \mathcal{O}(2)$. (In particular this implies that $d_{i} \geq \delta_{2}$ for all $i \in\{1,2,3\}$, by Lemma 6.5.)

It is then straightforward to check that at least one of the three statements in the above list must in fact be false: you cannot choose three pairs from each set without choosing all four pairs from at least one set. In other words, exactly two of the three statements in the above list are true.

Now consider the three pairs $\left\{A_{1}, B_{1}\right\},\left\{A_{2}, B_{1}\right\}$, and $\left\{A_{3}, B_{1}\right\}$. Suppose all three are in $\mathcal{O}(1) \cup \mathcal{O}(2)$. Then $\left\{A_{1}, B_{1}, A_{3}\right\}$ is of type $(1,1,2)$ or $(1,2,2)$, while $\left\{A_{1}, B_{1}, A_{2}\right\}$ and $\left\{A_{2}, B_{1}, A_{3}\right\}$ are each of type $(1,1,3),(2,2,3)$, or $(1,2,3)$. Note that if these last two triples are equivalent due to $g \in \pi_{1}(N)$, then $g$ must be parabolic fixing $B_{1}$, in which case $\lambda_{3}$ must intersect the arc from $B_{1}$ to $A_{2}$, a previous case. So assume these triples are not equivalent under $\pi_{1}(N)$. Then the three triples $\left\{A_{1}, B_{1}, A_{2}\right\},\left\{A_{2}, B_{1}, A_{3}\right\}$, and $\left\{A_{3}, B_{1}, A_{1}\right\}$ form a simpler geometric Mom-3 structure, which is torus-friendly unless $\left\{A_{1}, B_{1}, A_{2}\right\}$ and $\left\{A_{2}, B_{1}, A_{3}\right\}$ are both of type $(1,2,3)$. Note that this is only possible if $\left\{A_{1}, B_{1}\right\}$ and $\left\{A_{3}, B_{1}\right\}$ are in $\mathcal{O}(1)$ while $\left\{A_{2}, B_{1}\right\}$ is in $\mathcal{O}(2)$. Now note that $\left\{A_{j}, B_{2}\right\}$ must be in $\mathcal{O}(1) \cup \mathcal{O}(2)$ for at least one $j \in\{1,2,3\}$. If $j=2$, then $\left\{A_{2}, B_{1}, B_{2}\right\}$ is either a third (1,2,3)-triple (and not equivalent to either $\left\{A_{1}, B_{1}, A_{2}\right\}$ or $\left\{A_{2}, B_{1}, A_{3}\right\}$ since it shares an "edge" with both) or else it is of type $(1,1,3)$ or $(2,2,3)$; either way we get a geometric Mom-3 structure with either one or three triples of type $(1,2,3)$, which therefore is torus-friendly. Suppose $j=1$ or 3 ; by symmetry assume $j=1$. Then by our previous assumptions $\left\{A_{2}, B_{2}\right\}$ and $\left\{A_{3}, B_{3}\right\}$ must not be in $\mathcal{O}(1) \cup \mathcal{O}(2)$. Now we 
can apply Corollary 3.6 to the horoballs $A_{2}, A_{3}, B_{1}$, and $B_{2}$ to get

$$
\begin{aligned}
e_{3} e_{2}+e_{3} & \leq e_{3} e_{2} \cosh d_{2} \\
\Rightarrow d_{2} & \geq \cosh ^{-1}\left(1+e_{2}{ }^{-1}\right) .
\end{aligned}
$$

Note that $e_{2} \leq e_{3} \leq 1.5152$ then implies $d_{2}>0.9$, and hence by Lemma 6.11 one of $d_{1}, d_{3}$ must be less than $\delta_{2}$, contradicting our assumptions up to this point.

So assume at least one of $\left\{A_{1}, B_{1}\right\},\left\{A_{2}, B_{1}\right\},\left\{A_{3}, B_{1}\right\}$ is not in $\mathcal{O}(1) \cup \mathcal{O}(2)$, and similarly for $B_{2}$ instead of $B_{1}$. Now go back to the three statements listed above; we know exactly one of them is false. Suppose it is the first statement which is false and that the others are true (the other possibilities can be handled similarly). The only way to reconcile this with the sentence at the beginning of this paragraph is if $\left\{A_{1}, B_{1}\right\}$ and $\left\{A_{2}, B_{2}\right\}$ are not in $\mathcal{O}(1) \cup \mathcal{O}(2)$, or the same but with the roles of $B_{1}$ and $B_{2}$ reversed. But in either case, applying Corollary 3.6 to $A_{1}, A_{2}, B_{1}, B_{2}$ yields

$$
\begin{aligned}
e_{3}{ }^{2}+1 & \leq e_{3}^{2} \cosh d_{1} \\
\Rightarrow d_{1} & \geq \cosh ^{-1}\left(1+e_{3}{ }^{-2}\right) .
\end{aligned}
$$

Once more, if $e_{3} \leq 1.5152$, then this implies $d_{1}>0.9$, so by Lemma 6.11 one of $d_{2}$, $d_{3}$ must be less than $\delta_{2}$, contradicting our assumptions. This completes the proof in this case.

Case $13(\mathcal{O}(4)$-edge, $(1,1,4)$-triple $)$. The exact same argument as in case 2 applies, with the obvious modifications.

This, finally, completes the proof of Proposition 6.9, and in turn the proof of Theorem 6.1.

\section{TORUS-FRIENDly MOM- $n$ 's}

Having established Theorem 6.1, we now have an embedded cellular complex $\Delta$ corresponding to a geometric Mom- $n$ structure in the cusped manifold $N$. The next step in upgrading $\Delta$ to an internal Mom- $n$ structure of the type defined in [GMM2] is to prove the following:

Theorem 7.1. Suppose $N$ is a one-cusped hyperbolic 3-manifold with $\operatorname{Vol}(N) \leq$ 2.848 and suppose $\Delta$ is the embedded cellular complex corresponding to the geometric Mom-n structure produced by Theorems [5.9 and 6.1. Then the components of $N-\Delta$ each have torus boundary, or else there exists a simpler geometric Mom-n structure which is also of the type described in Theorems 5.9 and 6.1 .

Proof. From Theorem 5.9 we know that $n=2$ or 3 and that the geometric Mom- $n$ structure thus constructed is torus-friendly. Recall that this means either $n=2$, or $n=3$ and the Mom-3 structure does not have exactly two triples of type $(p, q, r)$, where $p, q$, and $r$ are distinct indices. We wish to prove that being torus-friendly implies that the boundary consists of a collection of tori.

Let $M$ be a thickened neighborhood of $\Delta$; note that by construction $\chi(\partial M)=$ $2 \chi(\Delta)=0$. If $\partial M$ consists of nothing but tori, then we're done. If $\partial M$ contains components which are not tori, then one of those components must be a sphere. Hence we wish to show that $\partial M$ does not contain any spherical components. Since $N$ is hyperbolic this is equivalent to showing that $N-\Delta$ does not have any components which are 3 -balls. 
Suppose $n=2$ and that one of the components of $N-\Delta$ is a 3-ball $B$. In the universal cover $\mathbb{H}^{3}, B$ lifts to a collection of 3-balls; choose one of them and call it $\tilde{B}$. The boundary of $\tilde{B}$ consists of two types of "faces". First, there are totally geodesic faces which are the lifts of 2-cells of $\Delta$ corresponding to triples. Note that no such 2-cell can possibly contribute more than two faces to $\partial \tilde{B}$. Second, $\tilde{B}$ has horospherical faces which are lifts of pieces of the cusp torus. There can be an arbitrary number of such faces, but each such horospherical face must only be adjacent to totally geodesic faces. (In particular the number of totally geodesic faces of $\tilde{B}$ must be greater than zero.) We can extrude $\tilde{B}$ in the direction of the horospherical faces (or equivalently, extrude $B$ in the direction of the cusp of $N$ before lifting to $\mathbb{H}^{3}$ ) to obtain an ideal hyperbolic polyhedron which contains $\tilde{B}$ and whose ideal triangular faces each contain a unique totally geodesic face of $\tilde{B}$.

But a geometric Mom-2 structure only has two triples, and hence $\tilde{B}$ cannot have more than four totally geodesic faces. Thus, the ideal polyhedron containing $\tilde{B}$ must be an ideal tetrahedron. But the same argument can be made for any component of $N-\Delta$ which is not the cusp neighborhood, and we've already used up all of the available totally geodesic faces. Therefore $B$ and the cusp neighborhood must be the only components of $N-\Delta$. This is impossible, since $\chi(\partial M)=0$. Therefore $N-\Delta$ cannot contain any 3 -ball components.

Now suppose $n=3$ and that one of the components of $N-\Delta$ is a 3 -ball $B$. As before, $B$ lifts to a 3 -ball $\tilde{B}$ in $\mathbb{H}^{3}$, which is in turn contained in an ideal hyperbolic polyhedron whose ideal triangular faces each contain a unique totally geodesic face of $\tilde{B}$. This time, there are six totally geodesic faces available; since a polyhedron with triangular faces must have an even number of faces, the number of totally geodesic faces of $\tilde{B}$ must be either 4 or 6 . If the number is 6 , then as before this implies that $B$ and the cusp are the only components of $N-\Delta$, which is impossible. Therefore $\tilde{B}$ has 4 totally geodesic faces and hence is contained in an ideal hyperbolic tetrahedron. This implies that $B$ is a truncated ideal hyperbolic tetrahedron, where the faces arising from the truncation are horospherical instead of geodesic.

Now consider the 2-cells in $\Delta$ which correspond to triples from the geometric Mom-3 structure. Since there are only three of them, some pair of faces of $B$ must arise from two sides of the same 2-cell. Suppose the corresponding triple is of type $(a, b, c)$ where $a, b$, and $c$ are distinct integers. The two faces of $B$ must share a common edge. Lifting up to $\mathbb{H}^{3}$, we see that $\tilde{B}$ has two geodesic faces which project down to the same 2 -cell in $\Delta$, and that these two faces have a common edge. There must be a group element $g \in \pi_{1}(N)$ which sends one face to the other and fixes the common edge, either fixing the horoballs at each end of the edge or swapping them. Therefore $g$ is the identity or is elliptic of order 2; either result is a contradiction.

Therefore the triple in question is of type $(a, a, b)$ for some distinct $a$ and $b$. (Lemma 3.2 excludes the possibility that it is of type $(a, a, a)$.) Again, the two corresponding faces must share a common edge. This edge cannot be the $\mathcal{O}(b)$ edge (as defined in Section 6) by the same argument as in the previous paragraph; hence the common edge must be an $\mathcal{O}(a)$-edge. Choose an orientation for this edge; i.e., make the corresponding 1-cell a directed arc. Lifting to $\mathbb{H}^{3}$ again, this induces an orientation on at least three of the edges of $\tilde{B}$, namely all of those edges which project down to the $\mathcal{O}(a)$-edge. There are now two possibilities; see Figure 9, First, all three of those edges may be oriented toward the same horospherical face of $\tilde{B}$, as 
(a)

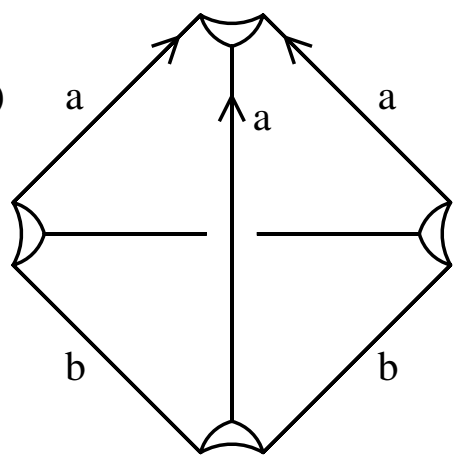

(b)

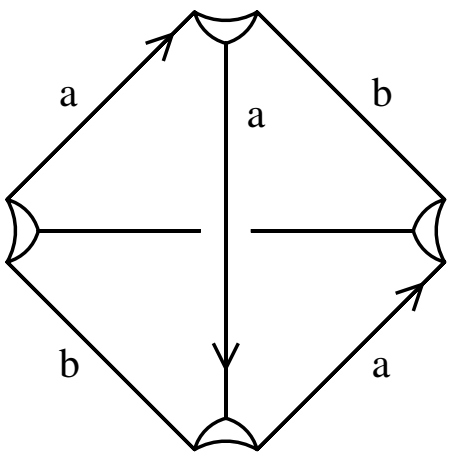

Figure 9. Configuration of the three-cell $\tilde{B}$, both (a) invalid and (b) valid.

in Figure 9(a). Note that there is a group element $g \in \pi_{1}(N)$ which sends one face of type $(a, a, b)$ to the other and that this group element preserves the orientation of the $\mathcal{O}(a)$-edges; therefore $g$ must be a parabolic element fixing the horosphere toward which those edges point. But a parabolic non-trivial element of $\pi_{1}(N)$ which takes a horosphere to itself must act on that horosphere by a translation. This would imply that all three edges labelled $a$ in Figure 9(a) lie in the same hyperbolic plane, and that $\tilde{B}$ therefore is flat, which contradicts our assumption that $\Delta$ was embedded in $N$.

The other possibility is that the two edges labelled $a$ in each of the $(a, a, b)$-type faces are oriented towards different horospherical faces. Since the two $(a, a, b)$-type faces share such an edge, such a configuration must look like Figure 9(b) (or like Figure 9(b) but with orientations reversed, which is handled identically).

In this situation, we have labelled all but one of the six geodesic edges of $\tilde{B}$; now we turn our attention to the last edge. Suppose that this edge projects down to the $\mathcal{O}(a)$-edge or the $\mathcal{O}(b)$-edge. Then at least two of the three triples in our Mom-3 only incorporate the orthopair classes $\mathcal{O}(a)$ and $\mathcal{O}(b)$; throwing away the third triple will leave us with a simpler geometric Mom-2 structure, as desired. Hence, suppose that the last edge projects down to the $\mathcal{O}(c)$-edge, where $c$ is the remaining index used in the Mom-3. Thus the other two geodesic faces of $\tilde{B}$ both project to 2-cells corresponding to triples of type $(a, b, c)$. Note that these last two faces cannot project down to the same 2-cell, since that would imply the existence of a non-trivial $g \in \pi_{1}(N)$ which is elliptic or the identity, just as before. Therefore the Mom- 3 contains exactly two triples of type $(a, b, c)$ where $a, b$, and $c$ are distinct, contradicting the assumption that the Mom-3 was torus-friendly. This proves the theorem.

It is worth pointing out that embedded geometric Mom-3's which are not torusfriendly, where some component of their complement is a 3-ball, do exist; we give an example below. It is also often the case that a manifold can have both a Mom-3 which is torus-friendly and a Mom-3 which is not. There are several hyperbolic manifolds which give rise to an embedded "Mom-like" cellular complex with three 


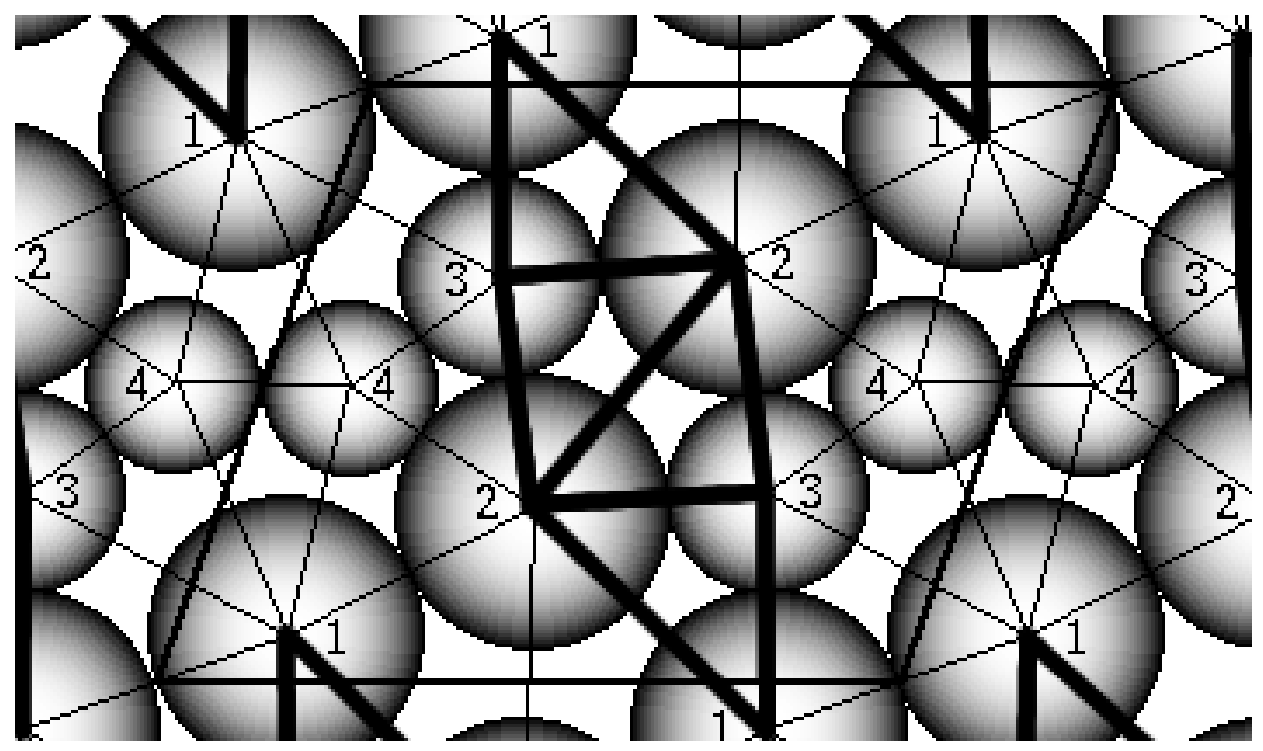

Figure 10. The manifold m170 with a Mom-3 structure which is not torus-friendly. The indices denote the orthoclasses of the horoballs.

1-cells and four 2-cells, such that two or three of the 2-cells are of type $(a, b, c)$. If one discards one of the four 2-cells, then the resulting geometric Mom-3 structure may or may not be torus-friendly depending on which 2-cell is discarded. It is easy to see, however, that if triples of type $(a, b, c),(a, b, c)$, and $(a, a, b)$ have already been found, then any possible fourth triple can be combined with some two of the first three triples to produce a Mom-3 which is torus-friendly.

As an example of a manifold containing a geometric Mom-3 which is not torusfriendly, consider the manifold known as m170 in the SnapPea census; a cusp diagram for this manifold is shown in Figure 10. The highlighted triangles in the diagram are all corners of the same ideal hyperbolic simplex. This simplex is bounded by only three faces and three edges in the triangulation of $\mathrm{m} 170$; those faces and edges together make up a cellular complex corresponding to a geometric Mom-3 structure, with one triple of type $(2,2,3)$ and two distinct triples of type $(1,2,3)$. This is therefore a geometric Mom-3 which is not torus-friendly. At the same time the interior of the highlighted simplex is a component of the complement of this cellular complex; i.e., there is a component of $N-\Delta$ which is a 3 -ball. Hence this geometric Mom-3 cannot be turned into a topological Mom-3 of the type described in GMM2. It is worthwhile to note, however, that this manifold does possess other geometric Mom-3 structures which are torus-friendly. Specifically there exists a fourth triple, of type $(1,1,2)$, which can be used to construct such a Mom-3.

\section{Fullness}

Based on the result of Theorem [7.1 throughout this section we assume that $N$ possesses a geometric Mom- $n$ structure where $n=2$ or 3 , that the corresponding 
cellular complex $\Delta$ is embedded, and that the components of $N-\Delta$ which are not cusp neighborhoods have torus boundary. At this point we switch from discussing cellular complexes to discussing handle structures as follows: thicken $T$ to $T \times I$ where $T \times 0$ faces the cusp, thicken each 1-cell of $\Delta$ to a 1-handle from $T \times 1$ to itself, and thicken each 2-cell of $\Delta$ to a 2-handle which runs over $T \times 1$ and three 1-handles counting multiplicity. The reason for this change in focus is solely to take advantage of the language and conclusions of GMM2. Specifically, $T \times I$ and the newly constructed 1-handles and 2-handles form a handle decomposition of a submanifold $M \subset N$. We will abuse notation and allow $\Delta$ to also refer to the handle decomposition of $M$; it should always be clear by the context whether we are referring to a cellular complex or a handle decomposition. By Theorem 7.1 we may assume that the boundary of $M$ is a collection of tori. Also, since $M$ is a subset of a hyperbolic manifold and contains both a cusp torus and a geodesic arc from the cusp torus to itself, $i_{*} \pi_{1}(M)$ cannot be abelian, where $i: M \rightarrow N$ is the inclusion map. In other words, $i: M \rightarrow N$ is a non-elementary embedding. Therefore $(M, T, \Delta)$ is a topological internal Mom- $n$ structure according to GMM2. As in GMM2, we will adopt the terminology of Matveev and refer to the intersection of the 1-handles (resp. 2-handles) with $T \times 1$ as islands (resp. bridges), and the components of the complement in $T \times 1$ of the islands and bridges will be called lakes. The valence of an island will be defined to be equal to the valence of the corresponding 1-handle, or equivalently the number of ends of bridges lying on the island. We assume that these valences are at least two; if any 1-handle has valence one, simply remove both it and the 2-handle adjacent to it to obtain a simpler Mom- $n$ structure.

Clearly each 1-handle in $\Delta$ contributes two islands while each 2-handle contributes three bridges. Suppose $\sigma$ is a 2 -handle corresponding to a triple of type $(a, a, b)$, where the type is defined as in Section 6. In other words some lift $\tilde{\sigma}$ of $\sigma$ in the universal cover of $N$ is adjacent to three horoballs $\{A, B, C\}$ such that the orthopairs $\{A, B\}$ and $\{B, C\}$ are in $\mathcal{O}(a)$ while $\{C, A\} \in \mathcal{O}(b)$. Let $a_{0}$ and $a_{1}$ denote the islands which are the endpoints of the 1-handle around the $\mathcal{O}(a)$-edge, and define $b_{0}$ and $b_{1}$ similarly. Then the intersection of $\tilde{\sigma}$ with $\partial B$ projects down to a bridge whose endpoints both lie on islands in the set $\left\{a_{0}, a_{1}\right\}$.

Definition 8.1. If this bridge described above joins $a_{0}$ to $a_{1}$, then we will say that $\sigma$ is a loxodromic 2-handle. If instead this bridge joins $a_{i}$ to itself for $i=1$ or 2 , then we will say $\sigma$ is a parabolic 2 -handle.

In either case there exists $g \in \pi_{1}(N)$ which sends $\{A, B\}$ to $\{B, C\}$ since those are in the same orthopair class; furthermore $g$ is uniquely defined. If $\sigma$ is a parabolic 2-handle, then we must in fact have $g(A)=C$ and $g(B)=B$ (i.e., $g$ is a parabolic group element), and the bridge from $a_{i}$ to itself must follow a straight closed path in the cusp torus which corresponds to $g$. Without loss of generality suppose $i=0$; i.e., suppose $a_{0}$ is joined to itself by a bridge. Then it is not hard to see that the other two bridges corresponding to $\sigma$ must join $a_{1}$ to $b_{0}$ and $b_{1}$, respectively. These bridges have equal length by Lemma 3.4 and the angle between the bridges at $a_{1}$ must equal the angle between the two bridge ends at $a_{0}$, since both angles are equal to the angle at which $\sigma$ meets itself along the $\mathcal{O}(a)$-edge. Clearly this angle is a straight angle at $a_{0}$, and therefore at $a_{1}$ as well. Thus the configuration of islands and bridges resulting from $\sigma$ is as in the left side of Figure 11. 

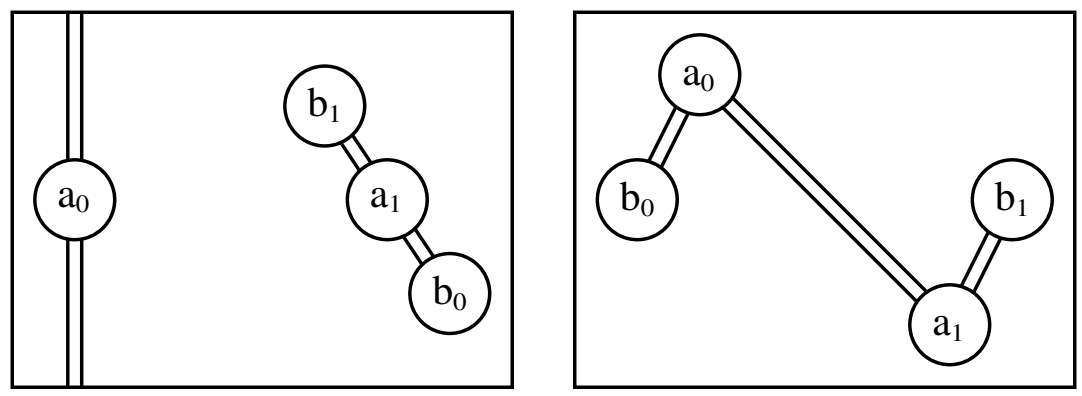

Figure 11. The islands and bridges belonging to a parabolic (left) and loxodromic (right) 2-handle. Note that the islands labelled $a_{0}$ and $a_{1}$ are endpoints of a single 1-handle (and similarly for $b_{0}$ and $\left.b_{1}\right)$.

If $\sigma$ is instead a loxodromic 2-handle, then we must have $g(A)=B$ and $g(B)=C$ (which implies that $g$ is a loxodromic group element although we will not prove that here). Again it is not hard to see that without loss of generality the other two bridges corresponding to $\sigma$ must join $a_{0}$ to $b_{0}$ and $a_{1}$ to $b_{1}$, respectively. Again, these two bridges must have the same length by Lemma 3.4, and the angle formed by the bridge ends at $a_{0}$ equals the angle formed by the bridge ends at $a_{1}$. Given that $N$ is orientable the configuration of islands and bridges arising from $\sigma$ must look like the right side of Figure 11.

The handle structure $\Delta$ is called full if all of the lakes are simply connected. If $\Delta$ is a full handle structure, then Theorem 4.1 of [GMM2] applies to $N$. Our goal in this section is to either prove that $\Delta$ is full or else construct a new topological internal Mom- $n$ structure in $N$ which is full. Lemma 4.5 of GMM2 does this in the case that $n=2$. Specifically it shows that a topological internal Mom-2 structure that is not full can be replaced with one which is. Hence we can combine Theorem 6.1. Theorem 7.1, Theorem 4.1 of [GMM2, and Lemma 4.5 of [GMM2] to conclude the following:

Proposition 8.2. If $N$ has a geometric Mom-2 structure, without loss of generality that structure can be thickened into a topological internal Mom-2 structure $(M, T, \Delta)$ that is both full and has torus boundary components. Consequently, $M$ is hyperbolic and $N$ can be recovered by Dehn surgery on $M$.

The assertion in the second sentence is one of the primary results of GMM2].

Unfortunately, the topological argument used in that proof does not extend easily to the $n=3$ case. Fortunately in this context we may take advantage of the fact that our Mom-3 is more than just a topological object; by construction the 1-handles and 2-handles of $\Delta$ have geodesic cores, an assumption that is not made in GMM2 . Call a Mom- $n$ structure with this additional property a geodesic internal Mom- $n$ structure. Our goal now is to prove the following:

Theorem 8.3. If $(M, T, \Delta)$ is an embedded geodesic internal Mom-3 structure in a hyperbolic manifold $N$ with torus boundary components, with 1-handles corresponding to the orthopair classes $\mathcal{O}(1), \mathcal{O}(2)$, and $\mathcal{O}(3)$, then $N$ has a full topological internal Mom- $k$ structure where $k \leq 3$. 
Proof. Suppose $\Delta$ is not full; i.e., suppose that $\Delta$ has one or more lakes which are not simply connected. The possible shapes for such a lake are the following: a torus with one or more holes, a disk with one or more holes, or an essential annulus with zero or more holes. In each case we wish to either find a contradiction or else construct a topological internal Mom-2 structure.

Suppose $T \times 1$ contains a lake which is a torus with holes. Then there is a simple closed loop $\gamma$ which bounds a disk in $T \times 1$ containing all of the islands and lakes. Push that disk into $T \times I$ to obtain a compressing disk for $M$ which separates $M$ into two pieces $M_{1}$ and $M_{2}$, such that $M_{1}$ is homeomorphic to $T \times I$ minus a 0 handle and $M_{2}$ consists of that 0-handle together with the 1-handles and 2-handles of $\Delta$. By assumption all of the boundary components of $M$ are tori; since $M$ is the connected sum of $M_{1}$ and $M_{2}$, and since $M_{1}$ has torus boundary, one of the boundary components of $M_{2}$ must be a 2 -sphere. Since $N$ is hyperbolic, the only possibility is that $M_{2}$ is contained inside a 3-ball. Therefore $M$ is the connected sum of $T \times I$ and a submanifold of $N$ contained inside a 3 -ball; this is impossible if $(M, T, \Delta)$ is geodesic.

Now suppose $T \times I$ contains a lake which is a disk with holes. Then let $\gamma$ be a simple closed curve parallel to the boundary of that disk, such that all of the islands and lakes inside the disk are also inside $\gamma$. Let $T_{0}$ denote the component of $\partial M$ which contains $\gamma$. As before, $\gamma$ bounds a disk in $T \times I$ which is a compressing disk for $M$ although it may not separate $M$. If it does separate $M$, then arguing as in the previous case we may show that the component of the separated manifold which does not contain $T \times 0$ is contained in a 3-ball in $N$, which is a contradiction if $(M, T, \Delta)$ is geodesic. So suppose that the compressing disk does not separate $M$; let $M_{1}$ be the manifold obtained after the compression. Note that the connectedness of $M_{1}$ implies that there must be a 1-handle in $\Delta$ with one endpoint inside $\gamma$ and one endpoint outside; this implies that $T_{0}-\gamma$ is connected, i.e. that $\gamma$ is essential in $T_{0}$. Hence the compression turns $T_{0}$ into a connected 2-sphere boundary component of $M_{1}$; call this sphere $S_{1} . M_{1}$ also has a handle structure $\Delta_{1}$ consisting of the 0 -handle carved out of $T \times I$ by the compressing disk, $T \times I$ minus that 0 -handle (which is homeomorphic to $T \times I$ ), and the 1-handles and 2-handles of $\Delta$. Now $S_{1}$ must bound a 3 -ball in $N$, and that 3-ball must lie on the outside of $M_{1}$ since $M_{1}$ contains $T \times 0$. Add this 3 -ball to $M_{1}$ as a 3 -handle to obtain a new manifold $M_{2} \subset N$ with torus boundary and new handle structure $\Delta_{2}$. Choose a 1-handle which connects the 0 -handle of $\Delta_{2}$ to $T \times I$; cancel that 1-handle with the 0-handle, and cancel the 3 -handle with a 2 -handle to obtain a handle structure $\Delta_{3}$ with only 1-handles and 2-handles.

We need to know that the embedding $i_{2}: M_{2} \rightarrow N$ is non-elementary, but note that $M_{2}$ actually contains $M$ : adding the 3 -handle to $M_{1}$ restores the portion of $M$ that was removed by the compression. So $i_{2}: M_{2} \rightarrow N$ is non-elementary since $i: M \rightarrow N$ is.

Now using the methods of GMM2, $\left(M_{2}, T, \Delta_{3}\right)$ can be simplified to obtain a new topological internal Mom- $k$ structure $\left(M_{4}, T, \Delta_{4}\right)$ on $N$. Since the construction of $\Delta_{3}$ deleted 1-handles and 2-handles without adding new ones, the complexity of $\left(M_{4}, T, \Delta_{4}\right)$ as defined in GMM2 must be strictly less than the complexity of $(M, T, \Delta)$; hence $k \leq 2$. Then by Lemma 4.5 of GMM2 we may assume that $N$ contains a full topological internal Mom- $k$ structure with $k \leq 2$. 
If $T \times I$ contains a lake which is an essential annulus with one or more holes, let $\gamma$ be a simple closed curve in the lake parallel to the boundary of one of those holes. Then proceed just as in the previous case.

The remaining possibility, and the one which will require the most analysis, is that $T \times 1$ contains lakes which are essential annuli without holes and lakes which are disks. The three 1-handles and three 2-handles generate six islands and nine bridges, which for Euler characteristic reasons imply that there must be three disk lakes and an unknown number of annulus lakes. Let $A_{1}, A_{2}, \ldots, A_{r}$ be the annulus lakes, and let $B_{1}, B_{2}, \ldots, B_{r}$ be the connected components of $T \times 1-\left(\bigcup_{i=1}^{r} A_{i}\right)$. Each $B_{i}$ is also an annulus, composed of islands, bridges, and disk lakes; furthermore each $B_{i}$ must contain at least one island.

Lemma 8.4. If $r>1$, then $N$ must contain a full topological internal Mom-2 structure.

Proof. Suppose $r=2$. Choose simple paths $\mu_{i}, i \in\{1,2\}$, such that $\mu_{i}$ crosses $B_{i}$ transversely for each $i, \mu_{i}$ does not cross any island for either $i$, and such that the total number of bridges crossed by $\mu_{1}$ and $\mu_{2}$ is minimal. Since there are only three disk lakes total in $B_{1}$ and $B_{2}$, the number of bridges crossed by $\mu_{1}$ and $\mu_{2}$ combined is at most five.

Slice $M$ open along $A_{1} \times I$ and $A_{2} \times I$ to obtain a new manifold $M_{1} \subset N$; note that $M_{1}$ will still have torus boundary and the inclusion $i_{1}: M_{1} \rightarrow N$ will still be non-elementary. $M_{1}$ consists of two thickened annuli, namely $B_{i} \times I$ for $i \in\{1,2\}$, and the 1-handles and 2-handles of $\Delta$. Each $\mu_{i}$ forms part of the boundary of a disk in $B_{i} \times I$; thicken each disk to obtain a decomposition of $B_{i} \times I$ into a 1-handle (the thickened disk) and a 0-handle (the complement of the thickened disk). Thus we obtain a standard handle decomposition $\Delta_{1}$ of $M_{1}$ consisting of two 0-handles, two new 1-handles (which we also refer to as $\mu_{1}$ and $\mu_{2}$ ), and the original 1-handles and 2-handles of $\Delta$. By construction the total valence of the new 1-handles $\mu_{1}$ and $\mu_{2}$ is at most five.

Now let $\lambda_{1}, \lambda_{2}$, and $\lambda_{3}$ denote the original 1-handles of $\Delta$, ordered in such a way that the valence of $\lambda_{1}$ is maximal. Note that without loss of generality we may assume that at least one of the $\lambda_{i}$ 's connects the two $B_{i}$ 's; otherwise we can simply throw away all of the 1-handles and 2-handles of $\Delta$ which are connected to $B_{1}$ to obtain a simpler geometric Mom- $n$ and apply Proposition 8.2. Suppose that $\lambda_{1}$ has both endpoints on $B_{1}$ (or equivalently on $B_{2}$ ). Then in $\Delta_{1}, \lambda_{1}$ has both endpoints on the same 0-handle. Drill out the core of $\lambda_{1}$ and this 0-handle to obtain a new torus boundary component $T_{2}$ and cancel the other 0-handle of $\Delta_{1}$ with one of the $\lambda_{i}$ 's which connects $B_{1}$ to $B_{2}$. The result is a new manifold $M_{2} \subset N$ and an internal topological Mom- $k$ structure $\left(M_{2}, T_{2}, \Delta_{2}\right)$, where $\Delta_{2}$ consists of the remaining $\lambda_{i}$, $\mu_{1}$ and $\mu_{2}$, and $T_{2} \times I$. Since the sum of the valences of the $\lambda_{i}$ 's equals 9 , either the valence of $\lambda_{1}$ was at least 4 and the valence of the cancelled $\lambda_{i}$ was at least 2 , or else the valence of all the $\lambda_{i}$ 's equals 3 . Either way the total valence of the two $\lambda_{i}$ 's removed to construct $\Delta_{2}$ is at least 6 , more than the total valence of the new 1-handles $\mu_{1}$ and $\mu_{2}$. Therefore the complexity of $\left(M_{2}, T_{2}, \Delta_{2}\right)$, as defined by GMM2, is less than the complexity of $(M, T, \Delta)$. Consequently $k \leq 2$. Then apply Lemma 4.5 of GMM2 to complete the proof. If $\lambda_{1}$ connects $B_{1}$ to $B_{2}$ but $\lambda_{2}$ had both endpoints on $B_{1}$, then drill out the core of $\lambda_{2}$ and one 0 -handle and cancel $\lambda_{1}$ with the other 0 -handle and then proceed as above. If every $\lambda_{i}$ connects $B_{1}$ to $B_{2}$, 
then drill out the cores of $\lambda_{1}, \lambda_{2}$, and both 0 -handles at the same time to construct $T_{2}$ and then proceed as above. This completes the proof in this case.

If $r=3$ the proof is similar. In this case we can construct $\mu_{1}, \mu_{2}$, and $\mu_{3}$ as paths which cross at most six bridges in total, then split along each $A_{i} \times I$ and turn the $\mu_{i}$ 's into new 1-handles with total valence at most 6 . The $B_{i}$ 's must all be connected by 1-handles; if $B_{1}$ were not connected to the others, then we could throw away all of the 1-handles and 2-handles of $\Delta$ connected to $B_{1}$ to obtain a simpler geometric Mom- $n$ and apply Proposition 8.2. Hence at most one of the $\lambda_{i}$ 's has both endpoints on the same $B_{i}$. Drill out the cores of enough $\lambda_{i}$ 's and 0handles to construct a new torus boundary component $T_{2}$ and cancel any remaining 1-handles with the remaining 0-handles to obtain an internal topological Mom- $k$ $\left(M_{2}, T_{2}, \Delta_{2}\right)$. Since the $\lambda_{i}$ 's have total valence 9 and the $\mu_{i}$ 's have total valence at most 6 , we have reduced complexity and hence $k \leq 2$. Then again apply Lemma 4.5 of GMM2.

If $r \geq 4$, then at least two $B_{i}$ 's contain only one island each. Since the bridges follow straight paths and since the $B_{i}$ 's are annuli, if $B_{i}$ contains only one island, then it also contains only one bridge. Such an island is the endpoint of a 1-handle $\lambda_{1}$ which is connected to only a single 2 -handle in $\Delta$; throw away the 1 -handle and the 2-handle to obtain a simpler geometric Mom- $n$ and then apply Proposition 8.2 This completes the proof of the lemma.

So suppose $r=1$. Let $A=A_{1}$ and $B=B_{1}$ for simplicity. We wish to use an argument similar to the one in the above lemma to obtain a topological Mom-2 structure. Specifically we wish to find a path $\mu$ which crosses $B$ transversely and which crosses no islands and as few bridges as possible. Suppose $\mu$ can be chosen to cross fewer than $v\left(\lambda_{1}\right)$ bridges, where $v\left(\lambda_{1}\right)$ is the valence of one of the 1-handles of $\Delta$. Then as in the lemma we can split $T \times I$ along $A \times I$, then decompose $B \times I$ into a 0 -handle and a 1 -handle, where the 1-handle is obtained by thickening a disk with $\mu$ in its boundary. Then by drilling out the cores of $\lambda_{1}$ and the 0 -handle we will obtain a topological internal Mom-2 structure which is full by Lemma 4.5 of GMM2.

Since $B$ contains only three disk lakes we can always choose $\mu$ to cross at most four bridges. Thus we're done if there exists a 1-handle with valence five or more. However this is not always the case. Let $\lambda_{1}, \lambda_{2}$, and $\lambda_{3}$ be the 1-handles of $\Delta$. We now consider the possible values for the valences of these three 1-handles. As previously stated the valence must add up to nine, so there are only three possibilities up to symmetry.

First suppose $v\left(\lambda_{1}\right)=5$ and $v\left(\lambda_{2}\right)=v\left(\lambda_{3}\right)=2$. Then we're done by the above argument.

Next, suppose $v\left(\lambda_{1}\right)=4, v\left(\lambda_{2}\right)=3$, and $v\left(\lambda_{3}\right)=2$. If we can find a path $\mu$ connecting the boundary components of $B$ which crosses no islands and no more than three bridges, then the usual splitting-and-drilling procedure will result in a strictly simpler internal Mom- $k$ structure. However it is not immediately apparent that such a path must exist; further analysis is required.

Let $\partial_{+} B$ and $\partial_{-} B$ denote the two boundary components of $B$. Consider the case where $\partial_{+} B$ contains exactly one island, and hence there is a bridge connecting that island to itself along a straight path in $T \times 1$. That bridge must be one corner of 
a parabolic 2-handle, as in the left side of Figure 11] The existence of a parabolic 2-handle turns out to have strong geometric consequence as follows:

Lemma 8.5. Suppose $\partial_{+} B$ contains exactly one island, and that consequently $(M, T, \Delta)$ contains a parabolic 2 -handle. If $(M, T, \Delta)$ contains a second parabolic 2-handle, then $N$ must have a full geodesic internal Mom-2 structure.

Proof. As before, if $\lambda$ is the 1-handle corresponding to $\mathcal{O}(a)$, then we will denote the islands at the end of $\lambda$ by $a_{0}$ and $a_{1}$ in some order. Also for brevity we will say that a 2-handle is "of type $(a, b, c)$ " if it corresponds to a triple of type $(a, b, c)$. A parabolic 2-handle is by definition of type $(a, a, b)$ for some $a$ and $b$; assume the island in $\partial_{+} B$ is the island $a_{0}$. If the second parabolic 2-handle is of type $(a, a, b)$ or $(b, b, a)$, then clearly we have a geometric Mom-2 structure which is necessarily full, torus-friendly, embedded, and so forth.

Suppose the second parabolic 2-handle is of type $(a, a, c)$, where $\mathcal{O}(c)$ is the remaining orthopair class in our Mom-3 structure. The only way this is possible is if the island $a_{1}$ is also connected to itself by a bridge. But then there would be two additional bridges which meet at the island $a_{0}$ at a straight angle, which is impossible if that is the only island in $\partial_{+} B$.

Suppose the second parabolic 2-handle is of type $(b, b, c)$. Then without loss of generality the island $b_{0}$ is also connected to itself by a bridge; since all of the bridges are contained in $B$, an annulus, the path this bridge follows must be in the same homotopy class as the bridge in $\partial_{+} B$. Since the bridges are geodesic, this means they must have the same length. Recall that if $e_{a}, e_{b}$, and $e_{c}$ are the elements of the Euclidean spectrum corresponding to $\mathcal{O}(a), \mathcal{O}(b)$, and $\mathcal{O}(c)$, then Lemma 3.4 implies that

$$
\frac{e_{c}}{e_{b}^{2}}=\frac{e_{b}}{e_{a}^{2}} \geq 1
$$

Hence $e_{c} \geq e_{b} \geq e_{a}$, and without loss of generality $c \geq b \geq a$. By the assumptions of Theorem 8.3 this implies that $a=1, b=2$, and $c=3$. Now consider the island $a_{1}$. While there is not necessarily a bridge connecting this island to itself, there is still a triple of horoballs corresponding to this island and the translation $g \in \pi_{1}(T)$ which acts in the direction of the annulus $B$. That triple must be of type $(a, a, k)$ for some $k$. Therefore Lemma 3.4 implies that

$$
\frac{e_{k}}{e_{a}^{2}}=\frac{e_{b}}{e_{a}^{2}} \text {. }
$$

So $e_{k}=e_{b}$. If $k \leq b=2$, then this implies that $N$ has a geometric Mom-2 structure. If $k \geq 3$, then $e_{k} \geq e_{3} \geq e_{2}$, i.e. $e_{3}=e_{2}$. But $e_{3} / e_{2}{ }^{2}=e_{c} / e_{b}{ }^{2} \geq 1$, so $e_{3}=e_{2}$ implies that $e_{3}=e_{2}=1$. This means that the horoballs centered at the islands $b_{0}$, $b_{1}, c_{0}$, and $c_{1}$ are all full-sized; i.e., in the upper half-space model they appear as Euclidean spheres of diameter 1 and are tangent to the horoball at infinity. Since there is a parabolic 2-handle of type $(b, b, c)$, by Lemma 3.4 the distance from $c_{0}$ and $c_{1}$ to $b_{1}$ is at most $1 / e_{c}=1$, and hence the horoballs centered at $c_{0}$ and $c_{1}$ abut the horoball at $b_{1}$. Similarly the length of the bridge from $b_{0}$ to itself is $e_{c} / e_{b}^{2}=1$, so the horoball at $b_{0}$ abuts itself; by symmetry, so does the horoball centered at $b_{1}$. Note that this implies that the shortest essential curve on the cusp torus has length at most one, so any full-sized horoball must abut itself. Finally the horoball at $b_{1}$ must abut the full-sized horoball centered at $a_{1}$, thanks to the parabolic triple of type $(a, a, b)$. This is a contradiction, as there is no way to arrange all of these full-sized horoballs around the island $b_{1}$ while keeping their interiors disjoint. 
If the second parabolic 2-handle is of type $(c, c, a)$, then permute the variables, replacing $c$ with $a$, $a$ with $b$, and $b$ with $c$. Then proceed as in the previous case.

The remaining possibility is that the second parabolic 2-handle is of type $(c, c, b)$. Without loss of generality the island $c_{0}$ is connected to itself by a bridge following a path in the same homotopy class (and hence of the same length) as the bridge in $\partial_{+} B$. Define $e_{a}, e_{b}$, and $e_{c}$ as before. Then

$$
\frac{e_{b}}{e_{c}^{2}}=\frac{e_{b}}{e_{a}^{2}} \geq 1
$$

Therefore $e_{b} \geq e_{c}=e_{a}$; hence $e_{2}=e_{1}$ and without loss of generality $a=1, c=2$, and $b=3$. Note that the length of the bridge connecting $a_{0}$ to itself must be $e_{3}$ by Lemma 3.4. Now consider the island $a_{1}$; it is connected by bridges to the islands $b_{0}$ and $b_{1}$, and these bridges both have length $1 / e_{3}$ and meet at $a_{1}$ in a straight angle. Similarly, the island $c_{1}$ is connected to $b_{0}$ and $b_{1}$ by bridges of length $1 / e_{3}$ which meet at $c_{1}$ in a straight angle. The only way this can happen inside the annulus $B$ is if the island $c_{1}$ lies exactly halfway along the shortest geodesic path from $a_{1}$ to itself, and vice versa. In other words, there are two geodesic paths of length $e_{3} / 2$ connecting $a_{1}$ to $c_{1}$. These paths correspond to triples of horoballs of types $(1,2, k)$ and $(1,2, l)$ for some $k$ and $l$ such that $e_{k}=e_{l}=e_{3} / 2<e_{3}$; clearly $k, l \in\{1,2\}$. These two triples cannot be equivalent under the action of $\pi_{1}(N)$; since they involve the same two islands, if $g \in \pi_{1}(N)$ mapped one triple to the other, then $g$ would also have to fix the cusp torus $T$, but the two bridges in question are clearly not equivalent under the action of $\pi_{1}(T)$. Therefore the triples $(1,2, k)$ and $(1,2, l)$ constitute a geometric Mom-2 structure. This completes the proof of the lemma.

So therefore we may assume that there is at most one island connected to itself by a bridge; in particular $\partial_{-} B$ contains more than one island.

Next consider a minimum-length sequence $\gamma=\left\{\iota_{0}, \beta_{1}, \iota_{1}, \ldots, \beta_{n}, \iota_{n}\right\}$ of islands and bridges such that $\iota_{0}$ is the sole island in $\partial_{+} B$ and $\iota_{n}$ is an island in $\partial_{-} B$. Note there are at most four bridges in the sequence (otherwise there would be more than six islands in total). If $\gamma$ contains only one or two bridges, it is straightforward to show that there must be a path $\mu$ from $\partial_{+} B$ to $\partial_{-} B$ lying in a small neighborhood of $\gamma$ which crosses at most three bridges. (Remember that at most two of the islands in the sequence $\gamma$ can have valence 4.) Suppose $\gamma$ contains four bridges; then together $\gamma, \partial_{+} B$, and $\partial_{-} B$ contain all six islands and at least seven bridges. If the remaining bridges are not placed in such a way that at least two bridge ends meet the islands in $\gamma$ on each side of the sequence (see Figure 12), then there will be a path $\mu$ crossing at most 3 bridges. So suppose there are at least two additional bridge ends on each side of the sequence; this implies that each of the two remaining bridges join an island in $\gamma$ to another island in $\gamma$ (and not, say, to the other island in $\partial_{-} B$ ). Now note that up to reordering of the indices there are only two combinations of triples which result in a Mom-3 structure which is torusfriendly and with these valences, and without containing a Mom-2 structure as a subset: triples of type $(a, a, b),(a, a, c)$, and $(b, b, c)$ for some ordering $\{a, b, c\}$ of the indices $\{1,2,3\}$, or triples of type $(a, a, c),(a, b, b)$, and $(a, b, c)$. There is no way to place the two remaining bridges that (a) preserves the minimality of $\gamma$, (b) doesn't imply the existence of a second parabolic 2-handle, (c) ensures two islands of each valence $\{2,3,4\}$, and (d) ensures that the number of bridges between each island 


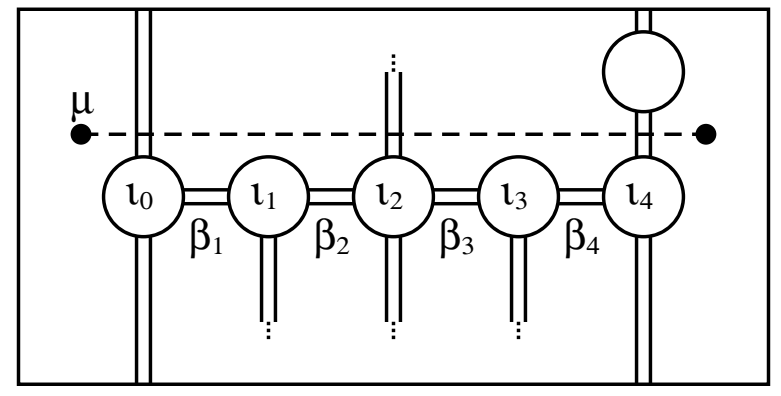

Figure 12. Finding a path $\mu$ from $\partial_{+} B$ to $\partial_{-} B$ which crosses at most three bridges.

matches the numbers produced by one of the two combinations of triples described above. If $\gamma$ contains 3 bridges, then $\gamma, \partial_{+} B$, and $\partial_{-} B$ contain at least five islands and at least six bridges. Again, if there are not two additional bridge ends on each side of $\gamma$, then we can find an appropriate path $\mu$; so suppose there are at least two additional bridge ends on each side. This implies that the missing island is of valence 2, since otherwise it would account for too many bridge ends. Again, there is no way to place an island of valence 2 and the three remaining bridges that satisfies the conditions (a), (b), (c), and (d) above.

Therefore if $\partial_{+} B$ contains exactly one island, then we can find a path $\mu$ from $\partial_{+} B$ to $\partial_{-} B$ crossing at most 3 bridges.

Suppose then that $\partial_{+} B$ and $\partial_{-} B$ each contain at least two islands. Define $\gamma$ as before; there are at most 3 bridges in the sequence. If there are only 1 or 2 bridges, then we can find $\mu$ crossing at most three bridges as before, so suppose $\gamma$ contains exactly 3 bridges. Then $\gamma, \partial_{+} B$, and $\partial_{-} B$ contain all six islands and at least seven bridges. Furthermore the two missing bridges must each join an island in $\gamma$ to another island in $\gamma$ or else there will be less than two additional bridge ends on one side of the sequence and hence a path $\mu$ crossing at most 3 bridges. But there is no way to place the two missing bridges that (a) preserves the minimality of $\gamma$, (b) ensures each missing bridge joins $\gamma$ to itself, (c) ensures two islands of each valence $\{2,3,4\}$, and (d) ensures that the number of bridges between each island matches the numbers produced by one of the two combinations of triples described earlier.

This completes the proof in the case where $v\left(\lambda_{1}\right)=4, v\left(\lambda_{2}\right)=3$, and $v\left(\lambda_{3}\right)=2$.

The remaining case is where $v\left(\lambda_{1}\right)=v\left(\lambda_{2}\right)=v\left(\lambda_{3}\right)=3$. Define $\partial_{+} B$ and $\partial_{-} B$ as before. If $\partial_{+} B$ and $\partial_{-} B$ each contain exactly one island, then Lemma 8.5 above shows that $N$ has a full internal Mom-2 structure. Suppose $\partial_{+} B$ and $\partial_{-} B$ each contain at least two islands. Consider the minimum-length sequence $\gamma=\left\{\iota_{0}, \beta_{1}, \iota_{1}, \ldots, \beta_{n}, \iota_{n}\right\}$ as before; $\gamma$ has no more than three bridges. If $\gamma$ has two or fewer bridges, then since each island has valence 3 there must be a path $\mu$ from $\partial_{+} B$ to $\partial_{-} B$ which crosses at most two bridges; split, thicken, and drill just as before to construct a topological internal Mom-2 structure. If $\gamma$ has exactly three bridges, then $\gamma, \partial_{+} B$, and $\partial_{-} B$ contain all six islands and at least seven bridges, but there is no way to place the missing two bridges that (a) preserves the minimality of $\gamma$ and (b) ensures all six islands have valence 3, except for configurations such as 


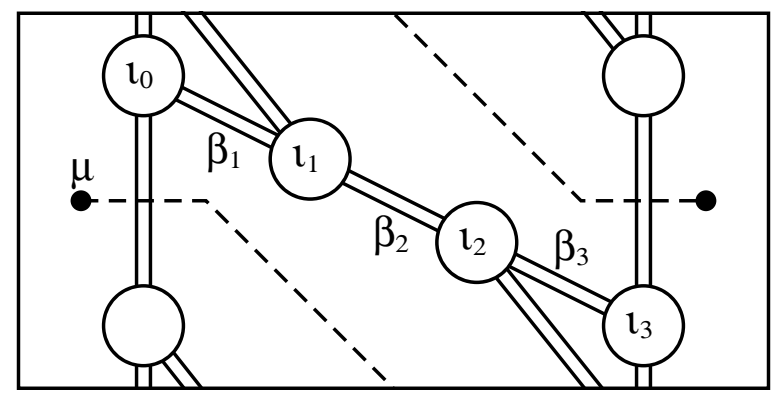

FiguRE 13. If each island has valence 3 and $\gamma$ has exactly three bridges, then we can always find a path $\mu$ crossing two bridges.

the one shown in Figure 13, in which there is clearly a path $\mu$ which crosses only two bridges.

So without loss of generality assume that $\partial_{+} B$ contains exactly 1 island and that $\partial_{-} B$ contains at least 2 . If $\partial_{+} B$ contains exactly one island, then the Mom3 structure must contain a parabolic 2-handle of type $(a, a, b)$ for some $a$ and $b \in$ $\{1,2,3\}, a \neq b$. We may assume the island in $\partial_{+} B$ is the one denoted $a_{0}$, and hence the islands $b_{0}$ and $b_{1}$ are each joined to $a_{1}$ by bridges which meet at $a_{1}$ in a straight angle. Consider the other two 2-handles in the Mom-3 structure; we may assume they are either of type $(b, b, c)$ and $(c, c, a)$, or of type $(c, c, b)$ and $(a, b, c)$, where $c$ is the remaining element of $\{1,2,3\}$, as no other combination produces 1-handles of the given valences without also including a geometric Mom-2 structure. Suppose the other two 2-handles are of type $(b, b, c)$ and $(c, c, a)$. If either of these 2-handles are parabolic, then we can apply Lemma 8.5. so suppose neither is parabolic. A loxodromic 2-handle of type $(b, b, c)$ implies that there is a bridge joining $b_{0}$ to $b_{1}$; this bridge together with the two-step path from $b_{0}$ to $a_{1}$ to $b_{1}$ must form a homotopically non-trivial loop in $B$. Also, without loss of generality the island $c_{i}$ is joined to $b_{i}$ by a bridge for $i=0,1$. Moreover since $(M, T, \Delta)$ is a geodesic structure, the angles subtended by the three bridges at $b_{0}$ must equal the angles subtended by the three bridges at $b_{1}$, albeit in opposite order since $N$ is orientable. Thus the bridges and islands must be arranged as in Figure 14, note in particular that $c_{0}$ and $c_{1}$ must be on opposite sides of the path $b_{0} \rightarrow a_{1} \rightarrow b_{1} \rightarrow b_{0}$. Now the third 2-handle, which is of type $c c a$ and which we are assuming is non-parabolic, must imply the existence of a bridge in $B$ which joins $c_{0}$ to $c_{1}$ without crossing any other bridge, which is clearly impossible.

Suppose then that the other two 2-handles in the Mom-3 structure are of type $(c, c, b)$ and $(a, b, c)$. Again, we may assume the 2-handle of type $(c, c, b)$ is a loxodromic 2-handle, not a parabolic one. Without loss of generality as in Figure 11 there exist bridges joining $b_{0}$ to $c_{0}, c_{0}$ to $c_{1}$, and $c_{1}$ to $b_{1}$. These bridges together with the bridges joining $b_{0}$ and $b_{1}$ to $a_{1}$ must again follow a homotopically nontrivial loop in $B$. In order to form such a loop in the annulus $B$, the angle at $b_{0}$ between the bridge to $a_{1}$ and the bridge to $c_{0}$ must equal the angle at $b_{1}$ between the bridge to $a_{1}$ and the bridge to $c_{1}$, and those angles must be in the same direction. But since $(M, T, \Delta)$ is a geodesic structure and since $M$ is orientable those angles must in fact be equal in the opposite direction as well. Hence those two angles are both straight angles; see Figure 15. The last 2-handle, of type $(a, b, c)$, implies that 


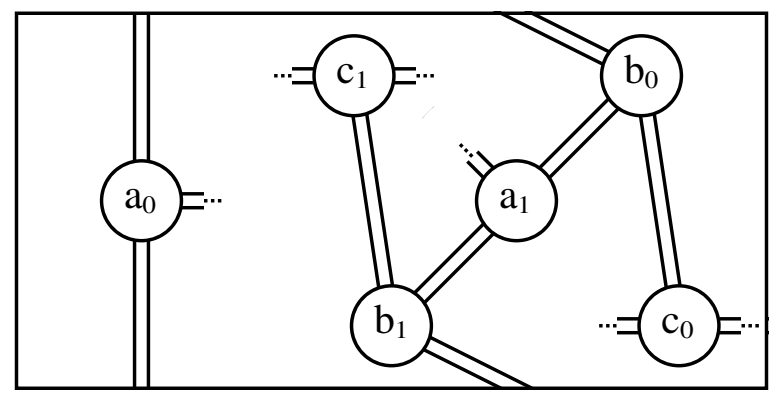

Figure 14. The islands and bridges corresponding to a parabolic 2-handle of type $(a, a, b)$ and a loxodromic 2-handle of type $(b, b, c)$. Note there is no way to include a loxodromic 2-handle of type $(c, c, a)$ as well.

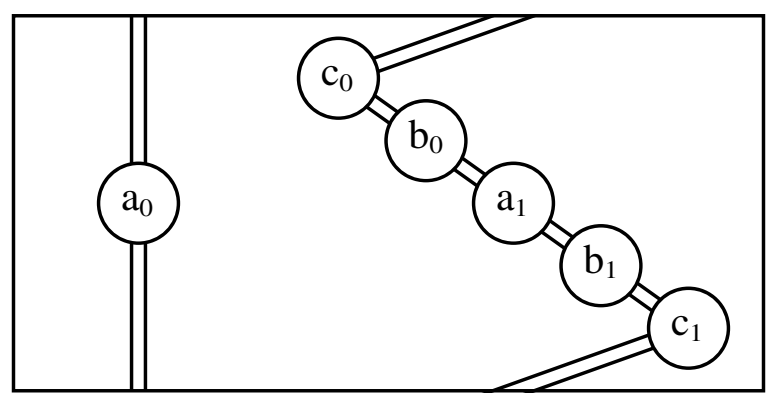

FiguRe 15. The islands and bridges corresponding to a parabolic 2-handle of type $(a, a, b)$ and a loxodromic 2-handle of type $(c, c, b)$. Note there is no way to insert another bridge from $a_{1}$ to any of the $b_{i}$ 's or the $c_{i}$ 's.

there are three more bridges: one joining $a_{i}$ to $b_{j}$ for some $i$ and $j$, another joining $b_{k}$ to $c_{l}$ for some $k$ and $l$ where $k \neq j$, and a third joining $c_{s}$ to $a_{t}$ where $s \neq l$ and $t \neq i$. In particular there must be a another bridge joining $a_{1}$ to one of $b_{0}, b_{1}, c_{0}$, or $c_{1}$, but there is no way to lay such a bridge without intersecting a preexisting bridge. This is a contradiction.

This completes the proof of the theorem.

\section{Proof of Theorem 1.1 and applichtions}

We begin by tying together the last three sections along with GMM2 and completing the proof of Theorem 1.1.

Suppose $N$ is a one-cusped hyperbolic 3-manifold with $\operatorname{Vol}(N) \leq 2.848$. Then by Theorem 5.9. $N$ contains a geometric Mom-2 or Mom-3 structure (which is not false), and by Theorem 6.1 we may assume that the corresponding cellular complex $\Delta$ is embedded in $N$. By Theorem[7.1, we may further assume that the components of $N-\Delta$ which are not cusp neighborhoods have torus boundary. Then by either Proposition 8.2 or Theorem 8.3 as appropriate, we may assume that the submanifold $M$ and handle decomposition $\Delta$ obtained by thickening the geometric Mom-2 or 
Mom-3 structure satisfy the definition of a full topological internal Mom- $k$ structure as given in GMM2.

Then by Theorem 4.1 of [GMM2 we can conclude that there exists a full topological internal Mom- $k$ structure $(M, T, \Delta)$ in $N$ where $k \leq 3$ and $M$ is hyperbolic. (Note this Mom- $k$ structure may bear little to no resemblance to the structure we started with!) This implies that $N$ can be recovered from $M$ by a hyperbolic Dehn filling on all but one of the cusps of $M$.

Finally Theorem 5.1 of GMM2 enumerates the possible choices for $M$. There are only 21 hyperbolic manifolds $M$ which can form part of a full topological internal Mom- $k$ structure $(M, T, \Delta)$ for $k \leq 3$, and these are precisely the manifolds listed in the table in Figure 1. This completes the proof of Theorem 1.1.

Theorem 1.1 does not in itself constitute an enumeration of all one-cusped manifolds with volume less than 2.848. However it is possible to analyze the Dehn surgery spaces of each of the 21 manifolds listed in Figure 1 We use the following theorem from [FKP]:

Theorem 9.1 (Futer, Kalfagianni, and Purcell). Let $M$ be a complete, finitevolume hyperbolic manifold with cusps. Suppose $C_{1}, \ldots, C_{k}$ are disjoint horoball neighborhoods of some subset of the cusps. Let $s_{1}, \ldots, s_{k}$ be slopes on $\partial C_{1}, \ldots$, $\partial C_{k}$, each with length greater than $2 \pi$. Denote the minimal slope length by $l_{\min }$. If $M\left(s_{1}, \ldots, s_{k}\right)$ satisfies the geometrization conjecture, then it is a hyperbolic manifold, and

$$
\operatorname{Vol}\left(M\left(s_{1}, \ldots, s_{k}\right)\right) \geq\left(1-\left(\frac{2 \pi}{l_{\min }}\right)^{2}\right)^{3 / 2} \operatorname{Vol}(M) .
$$

Therefore if $M$ is one of the two-cusped manifolds listed in Figure 1] and if we wish to enumerate all one-cusped manifolds with volume less than or equal to 2.848 that can be obtained by filling, then it is only necessary to examine surgery coefficients with slope less than or equal to

$$
2 \pi\left(\sqrt{1-\left(\frac{2.848}{\operatorname{Vol}(M)}\right)^{2 / 3}}\right)^{-1} .
$$

As an example, suppose $M$ is the Whitehead link complement, known as m129 in the SnapPea census. This manifold admits a symmetry which exchanges the cusps; therefore it does not matter which cusp we choose to fill in. (This is true for all of the manifolds listed in Figure1 1 except s785.) Using SnapPea, we see that the volume of m129 is $3.6638 \ldots$, which implies that we need only consider Dehn fillings along slopes of length less than 15.99. Given that a maximal cusp torus around one cusp has a longitude of length $2 \sqrt{2}$ and a meridian of length $\sqrt{2}$ at right angles to the longitude, we need only consider Dehn fillings with coefficients $(a, b)$, where $a$ and $b$ are relatively prime integers satisfying $2 a^{2}+8 b^{2} \leq 256$, clearly a finite and manageable number of cases. For each such filling, we can use SnapPea, Mos, and other such tools to confirm rigorously whether or not the resulting one-cusped manifold is hyperbolic and has volume less than 2.848 .

For s776, the only three-cusped manifold in Figure 1, we need to fill in two cusps to obtain a one-cusped manifold. However Theorem 9.1 only provides an upper bound on one of the corresponding slopes. Nevertheless, there are a finite number of possibilities for that one slope, and therefore filling in that one slope results in a 
finite number of two-cusped manifolds for which we can repeat the above analysis. Note that s776 admits symmetries which permute all of its cusps, so again it does not matter which cusps we fill. It should also be pointed out that Martelli and Petronio have already determined the complete list of fillings on s776 that result in non-hyperbolic manifolds (MP).

The results of the above analysis will be presented in detail in an upcoming paper $(\underline{M})$; for now we present the results of that analysis without proof:

Theorem 9.2. The only one-cusped orientable hyperbolic 3-manifolds with volume less than or equal to 2.848 are the manifolds known in the SnapPea census as m003, $m 004, m 006, m 007, m 009$, $m 010$, $m 011, m 015$, m016, and $m 017$.

Theorem 1.1 can also be used to analyze closed hyperbolic 3-manifolds. Lemma 3.1 of $\mathrm{ACS}$ states the following:

Lemma 9.3. Suppose that $M$ is a closed orientable hyperbolic 3-manifold and that $C$ is a shortest geodesic in $M$ such that $\operatorname{tuberad}(C) \geq(\log 3) / 2 . \operatorname{Set} N=\operatorname{drill}_{C}(M)$. Then $\operatorname{Vol}(N)<3.02 \operatorname{Vol}(M)$.

Here tuberad $(C)$ refers to the tube radius, i.e. the maximal radius of an embedded tubular neighborhood around $C$, while drill $C(M)$ is simply the manifold $M-C$ equipped with a complete hyperbolic metric. In other words, $N$ is a one-cusped hyperbolic 3-manifold from which the closed manifold $M$ can be recovered by Dehn filling. Two remarks are in order at this point. First, the Weeks manifold has volume less than 2.848/3.02 and is the smallest known closed orientable hyperbolic 3manifold. Second, according to GMT, if the condition that tuberad $(C) \geq(\log 3) / 2$ fails, then $M$ must have volume greater than that of the Weeks manifold anyway. Thus combining the above lemma with Theorem 9.2 yields the following:

Theorem 9.4. Suppose that $M$ is a closed orientable hyperbolic 3-manifold with volume less than or equal to that of the Weeks manifold. Then $M$ can be obtained by a Dehn filling on one of the 10 one-cusped manifolds listed in Theorem 9.2 .

Clearly we can use this result to identify the minimum-volume closed hyperbolic 3-manifold, using [FKP as before to limit the number of Dehn fillings that need to be considered. The results of such an analysis will also be presented in $[\mathrm{M}]$.

Of future interest is the problem of strengthening the bound of 2.848 in Theorem 1.1. and thus improving the classification of both closed and cusped low-volume hyperbolic 3-manifolds. The SnapPea census of cusped manifolds suggests that the number 2.848 should be far higher: the smallest known manifold which does not possess an internal Mom-2 structure is m069, which has a volume greater than 3.4. It would not be unreasonable to attempt to prove a stronger version of Theorem 1.1 which would apply to all one-cusped manifolds with volume less than or equal to 3.7 ; with such a result it would be possible to determine the first infinite string of volumes of one-cusped hyperbolic manifolds limiting on the volume of the Whitehead link complement. Similarly it should be possible to determine the first infinite string of volumes of closed manifolds limiting on the figure-eight knot complement.

Finally we expect that Mom-technology can be applied directly to closed manifolds, and not just indirectly via the use of Lemma 9.3 . The obstacles to this are primarily geometrical rather than conceptual. While the definition of a geometrical Mom- $n$ structure can easily be extended to closed manifolds (by considering triples of geodesics rather than triples of horoballs), difficulties arise when we consider the 
lessvol and overlapArea functions defined in Lemmas 3.8 and 3.10 In the cusped case the lessvol function uses the fact that the equidistant surface between two horoballs is a plane. In the closed case the equidistant surface between neighbourhoods of two geodesics is a more complicated surface. Similarly the overlapArea function uses the fact that the shadow of one horoball on the surface of another is a Euclidean circle; in the closed case, the shadow of one tubular neighbourhood on the surface of another is typically a not-quite-elliptical region whose shape depends on both the distance and the angle between the corresponding core geodesics. Developing a Mom-based theory for closed manifolds will require a more sophisticated analysis of these two geometrical problems.

\section{ACKNOWLEDGEMENT}

Some of this work was conducted while Gabai was a Member of the Institute for Advanced Study, while Meyerhoff was a Member of the Institute for Advanced Study and visiting professor at Princeton University and while Milley worked at Princeton University and at the University of California, Riverside. We greatly thank all these institutions for their hospitality.

\section{REFERENCES}

[Ada] Colin Adams, The Noncompact Hyperbolic 3-manifold of Minimum Volume, Proc. Amer. Math. Soc. 100 (1987), 601-606. MR894423 (88m:57018)

[ACS] Ian Agol, Marc Culler, and Peter Shalen, Dehn surgery, homology and hyperbolic volume, Algebr. Geom. Topol. 6 (2006), 2297-2312. MR.2286027 (2008f:57024)

[Ago] Ian Agol, Volume change under drilling, Geom. Top. 6 (2002), 905-916. MR.1943385 (2004e:57021)

[AST] Ian Agol, Peter Storm, and William Thurston, Lower bounds on volumes of hyperbolic Haken 3-manifolds, J. Amer. Math. Soc. 20 (2007), no. 4, 1053-1077. MR2328715 (2008i:53086)

[BPZ] S. Betley, J. Przytycki, and T. Zukowski, Hyperbolic structures on Dehn fillings of some punctured-torus bundles over $S^{1}$, Kobe J. Math. 3 (1986), no. 2, 117-147. MR.908780 (89f:57017)

[CM] Chun Cao and Robert Meyerhoff, The Orientable Cusped Hyperbolic 3-Manifolds of Minimum Volume, Invent. Math. 146 (2001), 451-478. MR1869847(2002i:57016)

[Fen] Werner Fenchel, Elementary Geometry in Hyperbolic Space, de Gruyter Studies in Mathematics 11, Berlin, 1989. MR,1004006 (91a:51009)

[FKP] David Futer, Efstratia Kalfagianni, and Jessica Purcell, Dehn Filling, Volume, and the Jones Polynomial, J. Differential Geom. 78 (2008), no. 3, 429-464. MR2396249 (2009c:57010)

[GMM] David Gabai, Robert Meyerhoff, and Peter Milley, Volumes of Tubes in Hyperbolic 3Manifolds, J. Differential Geom. 57 (2001), 23-46. MR1871490(2002i:57017)

[GMM2] David Gabai, Robert Meyerhoff, and Peter Milley, Mom Technology and Volumes of Hyperbolic 3-manifolds, arXiv:math.GT/0606072.

[GMT] David Gabai, Robert Meyerhoff, and Nathaniel Thruston, Homotopy hyperbolic 3-manifolds are hyperbolic, Annals of Math. 157 (2003), 335-431. MR:1973051 (2004d:57020)

[LM] M. Lackenby and R. Meyerhoff, The maximal number of exceptional Dehn surgeries, arXiv:0808.1176.

[MP] Bruno Martelli and Carlo Petronio, Dehn filling of the "magic" 3-manifold, Communications in Analysis and Geometry 14 (2006), no. 5, 967-1024. MR2287152 (2007k:57042)

$[\mathrm{MF}] \mathrm{S}$. Matveev and A. Fomenko, Isoenergetic surfaces of Hamiltonian systems, the enumeration of three-dimensional manifolds in order of growth of their complexity, and the calculation of the volumes of closed hyperbolic 3-manifolds, translated in Russian Math. Surveys 43 (1988), 3-24. MR937017 (90a:58052) 
[M] Peter Milley, Minimum volume hyperbolic 3-manifolds, Journal of Topology 2009, doi: 10.1112/jtopol/jtp006.

[M2] Peter Milley. Source code to produce and check the volume bounds discussed in Section 4 are available free of charge by e-mail from Milley.

[Mos] Harriet Moser, Proving a manifold to be hyperbolic once it has been approximated to be so, Algebr. Geom. Topol. 9 (2009), no. 1, 103-133. arXiv:math.GT/0809.1203. MR2471132

[P] Andrew Przeworksi, A universal upper bound on the density of tube packings in hyperbolic space, J. Differential Geom. 72 (2006), no.1, 113-127. MR2215457 (2007b:57030)

[Thu] William P. Thurston, Three-Dimensional Geometry and Topology, Princeton University Press, Princeton, 1997. MR 1435975 (97m:57016)

[W] Jeffrey Weeks, SnapPea, available from the author at www.geometrygames.org.

Department of Mathematics, Princeton University, Princeton, New Jersey 08544

Department of Mathematics, Boston College, Chestnut Hill, Massachusetts 02467

Department of Mathematics and Statistics, University of Melbourne, Melbourne, Australia 